\title{
High Conversion Efficiency Pumped- Cavity Second Harmonic Generation of a Diode Laser
}

D. M. Keicher

\author{
Prepared by \\ sendia National Laboratories \\ Abuquerque, Now Moxico 87186 and Luvermore, Callfornla 94550 \\ for the United states Department of Energy \\ undar Contrect DE-ACO4-04AL86000
}


Issued by Sandia National Laboratories, operated for the United States Department of Energy by Sandia Corporation.

NOTICE: This report was prepared as an account of work sponsored by an agency of the United States Government. Neither the United States Government nor any agency thereof, nor any of their employees, nor any of their contractors, subcontractors, or their employees, makes any warranty, express or implied, or assumes any legal liability or responsibility for the accuracy, completeness, or usefulness of any information, apparatus, product, or process disclosed, or represents that its use would not infringe privately owned rights. Reference herein to any specific commercial product, process, or service by trade name, trademark, manufacturer, or otherwise, does not necessarily constitute or imply its endorsement, recommendation, or favoring by the United States Government, any agency thereof or any of their contractors or subrontractors. The views and opinions expressed herein do not necessarily state or ref ct those of the United States Government, any agency thereof or any of thei: contractors.

Printed in the United States of America. This report has been reproduced directly from the best available copy.

Available to DOE and DOE contractors from Office of Scientific and Technical Information PO Box 62

Oak Ridge, TN 37831

Prices available from (615) 576-8401, FTS 626-8401

Available to the public from

National Technical Information Service

US Department of Commerce

5285 Port Royal Rd

Springfield, VA 22161

NTIS price codes

Printed copy: A07

Microfiche copy: A01 


\title{
HIGH CONVERSION EFFICIENCY PUMPED-CAVITY SECOND HARMONIC GENERATION OF A DIODE LASER
}

\author{
D. M. Keicher \\ Physical and Joining Metallurgy Department \\ Sandia National Laboratories \\ Albuquerque, New Mexico 87185
}

\begin{abstract}
To investigate the feasibility of producing a compact, efficient blue laser source, pumpedcavity second harmonic generation of diode lasers was explored. It is desirable to have such lasers to increase optical disk storage density, for color displays and for under-thesea green-blue optical signal transmission. Based on assumed cavity losses, a cavity was designed and numerical analysis predicted an overall conversion efficiency to the second harmonic wavelength of $76 \%$ from a $75 \mathrm{~mW}$ diode laser. The diode laser used in these experiments had a single longitudinal and a single transverse mode output at $860 \mathrm{~nm}$. The best conversion efficiency obtained (26\%) was less than optimum due to the $2.5 \%$ single-pass linear losses associated with the cavity. However, calculations based on these higher losses are in good agreement with the experimentally determined values. In additions, a factor of 1.65 increase in the second harmonic output power is anticipated by reducing the input mirror reflectivity to better impedance-match the cavity. With this relatively low second harmonic conversion, the power to light conversion is $7.8 \%$.
\end{abstract}

$$
\text { i - }
$$




\section{ACKNOWLEDGMENTS}

I would like to thank everyone who helped with the completion of this project. I would particularly like to thank my wife Jo Ann for the support that she has given me over the many years that we have been together and I have been in school and also Jim Jellison who has supported and encouraged me in my educational pursuits. Without their support, I would probably not be writing this thesis. I would also like to thank John McInerney for his support and guidance and for allowing me the opportunity to work under his direction. A special thanks goes to $\mathrm{Hua} \mathrm{Li}$ and Tim Lucas for providing insightful technical input in performing these experiments and also for providing assistance in understanding the experimental concepts. I also extend my appreciation to Malcolm Wright for the technical assistance he has provided during our discussions. I want to extend my appreciation to Greg Mizell of Virgo Optics who provided a second NLO crystal when the first crystal performance was less than optimum. I would like to thank my committee members Kevin Malloy and Wolfgang Rudolph for their support. I want to thank Jerry Knorovsky for reviewing my thesis and Phil Thacher for calibrating the laser power meter used in these experiments. Finally, I would like to extend iny appreciation to Mike Cieslak and Al Romig for allowing me to be on the "University Part Time" program at Sandia and again to Jim Jellison for his instrumental role in gaining permission for my acceptance into this program. 


\section{TABLE OF CONTENTS}

Chapter 1 INTRODUCTION

\section{Chapter 2 THEORY OF PUMPED-CAVITY SECOND HARMONIC} GENERATION

2.1 Gaussian Beam Propagation ....................................................... 8

2.2 Fabry-Perot Confocal Cavity ................................................... 14

2.3 Mode-Matching ........................................................................ 19

2.4 Second Harmonic Generation ..................................................... 23

2.5 Pumped-Cavity Second Harmonic Generation ............................... 28

2.6 Frequency-Locking ................................................................. 32

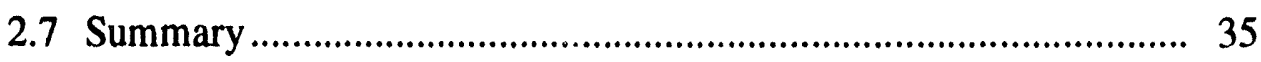

Chapter 3 SECOND HARMONIC GENERATION EXPERIMENTS ............ 38

3.1 Laser Beam Manipulation............................................................. 38

3.2 Laser Beam Parameter Measurements........................................... 40

3.3 Fabry-Perot Cavity Characterization ............................................ 48

3.4 Frequency-Locking ............................................................... 50

3.5 Single Pass Second Harmonic Generation ...................................... 53

3.6 Pumped-cavity Second Harmonic Generation................................ 57

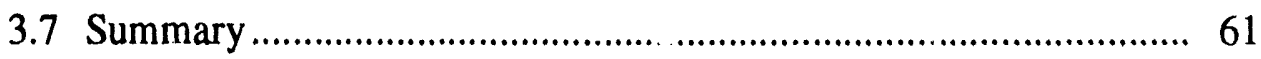

Chapter 4 EXPERIMENTAL RESULTS AND DISCUSSION .........................6 63

4.1 Laser Beam Parameters ................................................................... 63

4.2 Fabry-Perot Cavity Parameters ……............................................... 67

4.3 Frequency-Locking Results ..................................................... 75

4.4 Single Pass Second Harmonic Generation ................................... 79

4.5 Pumped-cavity Second Harmonic Generation................................. 82

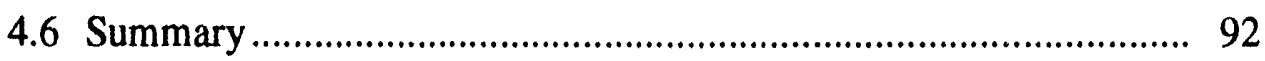

Chapter 5 SUMMARY AND CONCLUSIONS ……………........................ 95

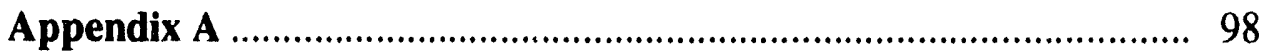

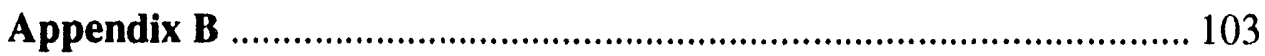

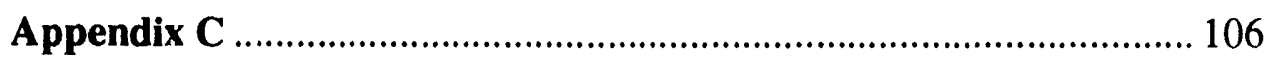

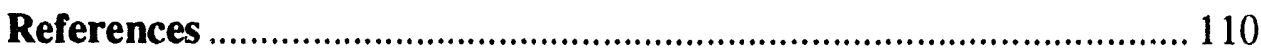




\section{LIST OF FIGURES}

Figure 1.1 Diagrammatic representation of intracavity SHG scheme for Nd:YAG laser

Figure 1.2 Diagrammatic representation of pumped-cavity SHG scheme for y,eneric laser input

Figure 2.1 Schematic view showing parameters used in defining Gaussian beam propagation

Figure 2.2 Gaussian beam propagation through a lens of focal lens $f$...

Figure 2.3 Schematic representation of real laser beam with the proposed embedded Gaussian beam contained within the real beam.

Figure 2.4 Confocal cavity configuration showing the mirror spacing to be twice the mirror focal length $f$ or, equivalently, at the radius of curvature of the mirrors.

Figure 2.5 Transverse mode frequencies for a confocal cavity. Note that the even symmetry transverse modes degenerate to positions indicated by $c / 2 R$ while the odd symmetry transverse modes degenerate to positions between these modes indicated by $\mathrm{c} / 4 \mathrm{R}$

Figure 2.6 Single transverse cavity mode excitation transmission characteristic as a function of cavity length $L$.

Figure 2.7 Schematic representation of geometry used to mode-match a beam of waist $w_{1}$ to a beam of waist $w_{2}$ using a single element lens

Figure 2.8 Configuration used in mode-matching a beam of a known waist $r_{1}$ to an apparent waist $w_{c}$ within a cavity

Figure 2.9 Polarization response in noncentrosymmetric crystal due an intense time-varying sinusoidal electric field (a) asymmetric polarization in NLO crystal, (b) polarization component at fundamental frequency $\omega$, (c) polarization component at SH frequency $2 \omega$, (d) dc component

Figure 2.10 (a) Block diagram for three-wave mixing process or sum frequency generation, (b) SHG is special case of sum frequency generation.

Figure 2.11 Schematic representation of pumped-cavity second harmonic generation configuration

Figure 2.12 Dither technique used to frequency-lock the diode laser wavelength to the cavity resonance peak

Figure 2.13 The expected error signal as the laser wavelength is scanned through the cavity resonance peak 
Figure 3.1 Simplified drawing of semiconductor diode laser showing differing divergence angles for the output laser beam

Figure 3.2 Schematic view of optical elements required to prepare the diode laser for the experiments leading up to and including the pumpedcavity second harmonic generation experiment

Figure 3.3 Effect of using anamorphic prism pair to increase the laser beam width in a single plane. Note that the beam width in the orthogonal plane in unaffected

Figure 3.4 Figure showing how optical feedback was introduced to diode laser to reduce the linewidth of the laser emission

Figure 3.5 Transmission characteristics from scanning Fabry-Perot interferometer as a function of time

Figure 3.6 Diagram showing scheme used in characterizing real laser beam to determine Gaussian beam parameters.

Figure 3.7 Geometry used to determine the apparent beam waist from the diode laser. Using this geometry, the previous optical elements can be ignored as if the beam were originating from a position at S.

Figure 3.8 Two geometries for the confocal cavities used in the experiments leading up to and including the pumped-cavity second harmonic generation, (a) off-axis confocal cavity, (b) normal incidence confocal cavity

Figure 3.9 Configuration used in experiments to develop a fundamental understanding of confocal cavities.

Figure 3.10 Block diagram of scheme used to frequency-lock the diode laser wavelength to the cavity length

Figure 3.11 Schematic representation of apparatus used in single pass SHG experiment.

Figure 3.12 Photograph of cell built to house $\mathrm{KNbO}_{3} \mathrm{NLO}$ crystal for temperature controlled phase-matching 56

Figure 3.13 Pumped-cavity second harmonic experiment configuration. 58

Figure 4.1 Measured transmission characteristics obtained from scanning 25 $\mathrm{cm}$ confocal resonator showing poor mode-matching condition. Note that the larger peaks correspond to excitation of the evensymmetry cavity transverse modes while the shorter peaks correspond to excitation of the odd-symmetry cavity transverse modes

Figure 4.2 Measured linewidth for Spectra Diode Labs model SDL-5422-H1 laser diode 
Figure 4.3 The uneven structure of the laser spectral distribution is evident when the cavity minimum resolution is three times less than the laser linewidth

Figure 4.4 Schematic representation of geometry used to develop modematching conditions of a laser beam to a cavity both of which have known parameters

Figure 4.5 Measured transmission characteristics of $5 \mathrm{~cm}$ confocal cavity with extremely good mode-matching between incident laser beam and the cavity

Figure 4.6 Reflection from scanning cavity with cavity losses varied: (a) empty cavity (b) glass slide inserted into cavity

Figure 4.7 Calculated reflectance from the $5 \mathrm{~cm}$ confocal cavity as a function of the cavity reflectance parameter....

Figure 4.8 Graph showing measured transmitted (1) and reflected (2) signals for frequency locking of laser diode to cavity resonance

Figure 4.9 Measured transmission characteristics from the pumped-cavity while frequency-locking to the cavity was maintained using feedback control

Figure 4.10 Graph showing measured transmission signal from cavity (1) when the cavity is detuned to be slightly off resonance and the corresponding error signal (2)

Figure 4.11 Photograph of equipment used in the frequency-locking the diode laser to the pumped-cavity

Figure 4.12 Photographs of pumped-cavity SHG experiment: (a) complete experiment configuration, (b) diode laser output beam manipulation and isolation optics, (c) cavity with NLO crystal, (d) optics used in SHG power and transmitted intensity measurements

Figure 4.13 Measured unidirectional SH output power from pumped-cavity SHG experiments shown with predicted SH output power expected from the cavity. Differential error analysis was performed on the calculated values to generate error.

Figure 4.14 Measured beam radii for the SH beam output from the pumpedcavity

Figure 4.15 Measured SHG at various power levels and temperatures used to determine the phase-matching temperature bandwidth 


\section{LIST OF TABLES}

Table 4.1 Measured values for real beam radii from the diode laser after optical manipulation to circularize the beam

Table 4.2 Calculated values for the focused laser beam and the apparent beam incident on the lens which produced focused beam values 68

Table 4.3 Calculated values for mode-matching to $5 \mathrm{~cm}$ confocal cavity 70

Table 4.4 Statistical values obtained for analysis of the frequency-locked beam behavior

Table 4.5 Measured values obtained from the single pass SHG experiment 81

Table 4.6 Calculated beam quality for SH laser light output from pumpedcavity experiment

Table 4.7 Phase-matching temperature bandwidth determined graphically from experimental results 


\section{LIST OF SYMBOLS}

\begin{tabular}{ll}
$\mathrm{M}_{1}, \mathrm{M}_{2}$ & Cavity front and rear mirrors \\
$w_{o}$ & Gaussian beam minimum waist \\
$\lambda$ & Laser wavelength \\
$z_{R}$ & Gaussian beam Rayleigh range \\
$\pi$ & 3.1416 \\
$w(z)$ & $\begin{array}{l}\text { Gaussian beam radius at a position } \mathrm{z} \text { with respect to minimum beam waist } \\
\text { location }\end{array}$ \\
$\boldsymbol{\theta}$ & Half-angle divergence of laser beam \\
$S$ & Position along the beam optical axis taken with respect to $w_{o}$ location \\
$S^{\prime}$ & Distance from lens to beam waist \\
$z_{R}^{\prime}$ & Distance from lens to transformed beam waist \\
$f$ & Rayleigh range for transformed beam \\
$W_{o}$ & Lens focal length \\
$\Theta$ & Real beam waist radius \\
$M$ & Real beam half-angle divergence \\
$R$ & Real beam propagation factor \\
$\Delta f_{a x}$ & Mirror radius of curvature \\
$c$ & Axial mode spacing \\
$\Delta v$ & Speed of light in a vacuum \\
$R_{1}$ & Cavity free spectral range \\
\hline$v_{1 / 2}$ & FWHM bandwidth for the cavity resonance peaks \\
\hline & Cavity finesse \\
\hline & Mirror power reflectivity for symmetrical cavity \\
\hline & Cavity input mirror coupling coefficient \\
\hline &
\end{tabular}


$\delta_{2} \quad$ Cavity output mirror coupling coefficient

$R_{i}, r_{i} \quad$ Generic mirror power reflectivity and reflection coefficient respectively

$f_{o} \quad$ Mode-matching characteristic length

$w_{1}, w_{2} \quad$ Mode-matching beam waists

$d_{1}, d_{2} \quad$ Waist positions with respect to mode-match lens

$w_{c} \quad$ Apparent beam waist within cavity for mode-matching

$w_{L} \quad$ Beam radius within cavity at mirror surface

$d_{L} \quad$ Overall cavity length to outside of mirrors

$z_{L} \quad$ Location of apparent beam waist with respect to outer mirror surface

$n \quad$ Index of refraction

$\mathbf{P}(t) \quad$ Polarization vector

$\varepsilon \quad$ Optical material permittivity

$\chi^{(1)} \quad$ Optical material linear susceptibility

$\mathbf{E}(t) \quad$ Electric field vector

$\chi^{(2)}, \chi^{(3)} \quad$ Second- and third-order linear susceptibilities

$\omega_{1}, \omega_{2}, \omega_{3} \quad$ Frequencies due to sum frequency generation process

$t \quad$ Time

$\mu \quad$ Permeability

$\Delta k \quad$ Difference between propagation constants of the fundamental and SH wavelengths

$k^{(2 \omega)}, k^{(\omega)} \quad$ Propagation constants for the SH and fundamental wavelengths

$P_{2 \omega}, P_{\omega} \quad$ Power of SH and fundamental beams

$l \quad$ NLO crystal length

$z \quad$ Distance along beam optical axis

A Beam cross-sectional area 
SH conversion efficiency

$n(\omega), n(2 \omega)$ Index of refraction of NLO crystal at fundamental and SH frequencies

$R_{1}, R_{2} \quad$ Confocal cavity input and ontput mirror power reflectivities

$T_{1}, T_{2} \quad$ Confocal cavity input and output mirror power transmission

$T_{S H} \quad$ Cavity transmission due to $S \mathrm{H}$ conversion

$\gamma_{S H} \quad$ Nonlinear conversion factor

$P_{c} \quad$ Circulating fundamental power within the cavity

$R_{m} \quad$ Cavity reflectance parameter

$T \quad$ Cavity transmission due to linear losses

$P_{\text {in }} \quad$ Incident power onto the cavity input coupler

$L \quad$ Cavity round trip length

$\Delta v(t) \quad$ Measured free spectral range as a function of time

$\Delta v_{1 / 2}(t) \quad$ Measured FWHM value for cavity resonance peak as a function of time

$W_{1}, W_{2} \quad$ Measured real beam radii

$z_{1}, z_{2} \quad$ Position along optical axis for real beam radii measurements

$\Delta \lambda \quad$ Small change in wavelength

$\Delta \phi \quad$ Phase difference between modulation signal and detected signal

$P_{I R} \quad$ Measured fundamental power into the NLO crystal

$P_{I R+b l u e} \quad$ Measured total power out of the NLO crystal

$P_{\text {IRprism }} \quad$ Measured fundamental power output from prism

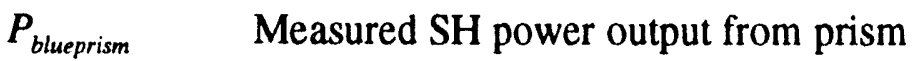

$d$

Nonlinear optical coefficient of SHG crystal 


\section{Preface}

The work reported in this document was performed at the University of New Mexico as partial fulfillment for a Masters degree in Electrical Engineering. The experiments were performed while enrolled in the University Part Time program at Sandia and most of the equipment used was Sandia property. The association with the University proved to be a very benificial experience. 


\section{CHAPTER 1}

\section{Introduction}

For applications such as color displays, optical disk storage, color laser printing, under water optical signal transmission, etc., it is desirable to have a compact blue laser source. For optical disk storage, the shorter wavelength of blue lasers provides a smaller focused spot and thus allows for higher density storage. Argon lasers do have a blue emission line at $488.5 \mathrm{~nm}$; however, these lasers are terribly inefficient $(<0.1 \%)^{[1]}$ and are very large physically. It is much more desirable to have a source with properties similar to semiconductor diode lasers. Although diode lasers are both compact and efficient, the difficulties associated with producing wide bandgap semiconductor materials have forced alternative methods to be investigated. To overcome this problem associated with diode lasers, nonlinear optical (NLO) techniques have been employed, particularly secondharmonic generation (SHG). The SHG frequency conversion process has allowed infrared output of solid-state lasers to be efficiently converted to the $\mathrm{SH}$ wavelengths. Several methods have been employed to produce SHG with varying degrees of success. The most efficient technique reported to date ${ }^{[2]}$ is a technique known as pumped-cavity SHG and was chosen as the method to investigate the feasibility of producing an efficient blue laser semiconductor source.

Semiconductor diode lasers possess several properties which make them highly desirable for applications which require compact, coherent light sources. These devices are grown from III-V type semiconductor materials and can be much less than a millimeter ${ }^{[3]}$ in any dimension. Although these devices are small, they can be made to produce tens or even hundreds of $\mathrm{mW}$ with near diffraction limited output beam quality and with efficiencies greater than $50 \%$. In addition, since these devices are semiconductors, they can be mass 
produced and their output wavelength can be tailored during the semiconductor growth operation and possess very long operating lifetimes. It is these advantages that have allowed the semiconductor diode laser to gain wide spread acceptance and applications requiring such a source to be brought to fruition.

The phenomenon of frequency conversion of light, and particularly SHG of laser light, was first demonstrated more than three decades ago[4]. When the laser light propagates through a nonlinear optical (NLO) material, the time varying electric field associated with the laser beam gives rise to a time-varying polarization within the crystal. Since NLO crystals lack an inversion symmetry, a directionally dependent restoring force on the electrons exists leading to an asymmetric polarization. It is this asymmetric time varying nonlinear polarization that leads to frequency conversion of the original electric field. For a given NLO crystal, the nonlinear conversion efficiency is related to the strength of the electric field (i.e. to the peak optical intensity) propagating through the crystal and the crystal length. In general, the useful length of NLO crystals is limited to $1-2 \mathrm{~cm}$ by either interference effects within the crystal (i.e. walk-off) or crystal growth limitations. Thus, high efficiency, single-pass SHG is obtainable only with laser sources possessing large electric fields, i.e. high peak intensities. This is, of course, subject to satisfying a phasematching condition between the SH and fundamental beams within the crystal. These high peak intensities are usually only obtainable from very short pulse lasers. To overcome the limiting requirement for high peak intensity due to crystal length constraints, innovative techniques have been used to obtain efficient SHG from low peak intensity continuous wave $(\mathrm{CW})$ lasers. These techniques have taken advantage of the power enhancement provided inside optical resonators. The two most widely used techniques are: intracavity and pumped-cavity SHG. These techniques have allowed efficient SHG to be achieved with low peak power CW lasers. 
The most widely used of these techniques is intracavity SHG[5]. A diagram depicting an intracavity SHG scheme is shown in Figure 1. The cavity is composed of two mirrors, $\mathbf{M}_{1}$ and $\mathbf{M}_{2}$, which provide optical feedback through a gair medium, such as an optically pumped Nd:YAG rod. For a laser cavity, in general, the cavity rear mirror, $M_{1}$, will be coated to have a maximum reflection at the fundamental laser wavelength while the cavity front mirror will be partially transmitting to allow a portion of the circulating beam within the cavity to leak out and provide an output from the laser cavity. However, for intracavity SHG both mirrors are coated to have a maximum reflection at the laser fundamental wavelength to maximize the circulating intensity within the laser cavity and the output mirror, $\mathrm{M}_{2}$, is coated for maximum transmission of the $\mathrm{SH}$ light. A polarizer is usually included in the laser cavity to enhance the laser gain preferentially in one polarization direction. Since SHG is polarization dependent, polarization control is required for phase-matching. To perform the parametric process of SHG, the NLO crystal is placed in the laser cavity. Mode limiting apertures can also be included in the cavity to improve the beam quality.

As previously mentioned, single pass SHG for CW lasers is inefficient due to the relatively small electric field amplitude provided by the low intensity beam. By placing the NLO crystal in the laser cavity, the electric field amplitude of the laser is effectively increased since the laser output intensity is a small fraction of the circulating intensity within the laser cavity. This technique has been proven over time and provides a reliable method for producing green light efficiently from a low peak intensity $\mathrm{Nd}$ :YAG laser. CW SH power outputs of $9 \mathrm{~W}$ have been demonstrated using this technique[6]. One limitation to this method is that the laser beam characteristics are degraded by placing the SHG crystal within the laser cavity. Although this method works well for the solid state $\mathrm{Nd}$ :YAG laser, this technique is not generally effective for producing SHG from diode lasers since the cavity mirrors are an integral part of the semicinductor diode laser. 


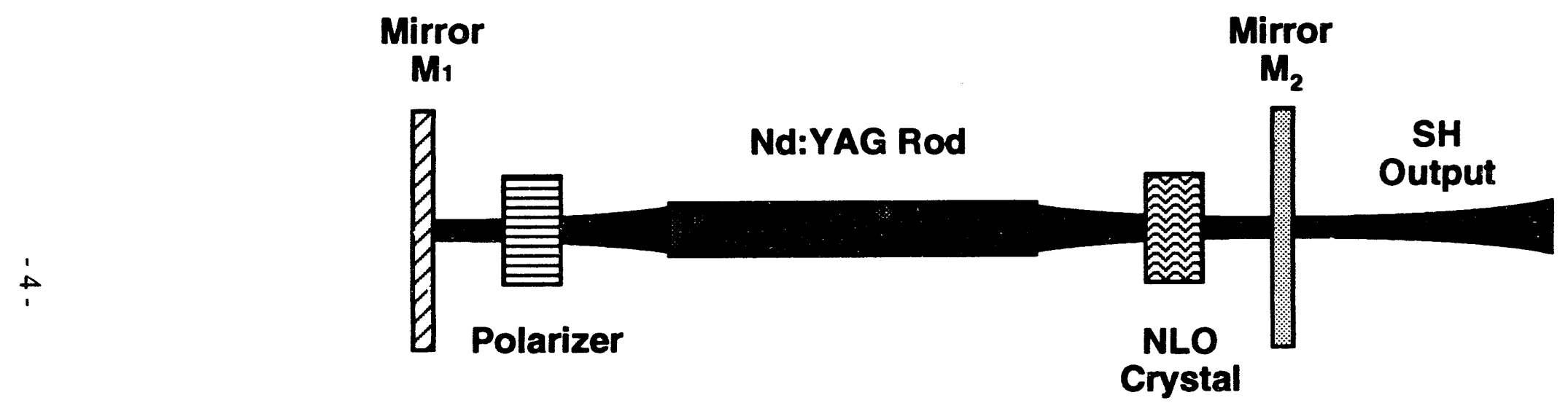

Figure 1.1 Diagramatic representation of intracavity SHG scheme for Nd:YAG laser. 
Another method used to produce SHG efficiently from low peak intensity sources is pumped-cavity SHG techniqu [7]. This method, shown in Figure 2, also utilizes the intensity enhancement provided by a resonant cavity. In this configuration, a laser with the desired wavelength for frequency conversion is used to pump a second cavity. The cavity is composed of two mirrors, $\mathbf{M}_{1}$ and $\mathbf{M}_{2}$, and the NLO crystal is placed within the cavity. To avoid unwanted optical feedback to the pump laser, the laser cavity is isolated, either optically or geometrically, from the pumped-cavity containing the NLO crystal. Generally, the beam from the pump laser is mode-matched to the pumped-cavity fundamental mode. The pumped-cavity output mirror $\mathbf{M}_{2}$ is a high reflector for the fundamental wavelength and anti-reflection coated at the SH wavelength to contain the laser fundamental wavelength within the cavity and transmit the SHG light. The cavity input coupler $M_{1}$ is coated to have a reflection at the fundamental laser wavelength that matches the losses in the cavity due to absorption, scattering and the SHG process. When the reflection of the input coupler matches these cavity losses and the cavity is resonant, then the reflected energy is minimized. When the cavity resonant condition is satisfied, the circulating intensity within the cavity is increased significantly from the normal output intensity level of the laser. The SHG beam output mode is dependent on the pumped-cavity resonant mode. This method allows the pump laser characteristics to be optimized independently of the SHG pump cavity. Spectrally pure, single frequency operation can also be obtained using this method. One drawback of this technique is that care must be taken to match the pumped-cavity length to the laser cavity length. Using this technique, an output power of $6.5 \mathrm{~W}$ of SH light has been obtained from a pump laser power of $18 \mathrm{~W}^{[8]}$ for a $\mathrm{Nd}$ :YAG laser source. In addition, pumped-cavity SHG has provided $54 \mathrm{~mW}$ output with a fundamental wavelength input power of $125 \mathrm{~mW}$ [9] using an $856 \mathrm{~nm}$ diode laser as a pump source. 


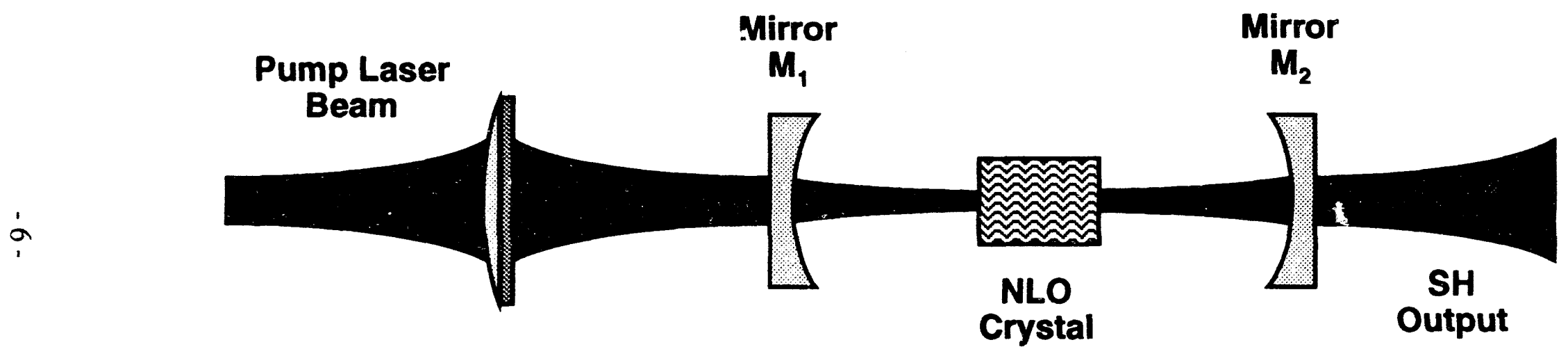

Figure 1.2 Schematic representation of pumped cavity SHG configuration for generic laser input. 
Both intracavity and pumped-cavity techniques have been demonstrated which provide efficient SHG. The intracavity technique provides a simpler technique to achieve a low peak intensity SHG and several low peak intensity pulsed systems are commercially available which utilize this s heme[10]. More recently, the desire for compact, single frequency visible light sources based on diode lasers has motivated researchers to investigate the punped-cavity scheme. Although more complicated, the pumped-cavity SHG technique provides a physically realizable solution to the problem of SHG based on diode lasers and allows optimization of the pump laser beam characteristics to be independent of the SHG process.

Although a conversion efficiency of $43 \%$ has been previously obtained ${ }^{[9]}$, initial calculations based on a $0.4 \%$ single pass loss within the cavity indicate that a conversion efficiency of $76.5 \%$ can be obtained. This conversion efficiency is nearly twice that of the previously reported results. Thus experiments were performed based on these calculations to obtain this extremely high conversion efficiency from a low power $\mathrm{cw}$ semiconductor laser diode source. 


\section{CHAPTER 2}

\section{Theory of Pumped-Cavity Second Harmonic Generation}

\subsection{Gaussian Beam Propagation}

In the limiting case of the fundamental spatial mode, laser beams can be considered to be Gaussian beams. From diffraction theory[11], the behavior of diffraction limited Gaussian beams upon propagation through an optical system has been well defined. There are several inter-related parameters used to describe Gaussian beams and their propagation (see Fig. 2.1). The minimum radius (or waist $w_{o}$ ) of the beam is defined to be the radial position where the electric field has decreased to a value 1/e times (or equivalently where the intensity has decreased by a factor $1 / \mathrm{e}^{2}$ ) the maximum on-axis value and the beam phase front is plane. The minimum waist is given by

$$
w_{o} \equiv \sqrt{\frac{\lambda z_{R}}{\pi}}
$$

where $\lambda$ is the wavelength of the laser and $z_{R}$ is the Rayleigh range of the beam. The Rayleigh range is defined to be the distance between the waist location and the location where the beam radius $w(z)$ increases to

$$
w(z)=\sqrt{2} w_{o} .
$$

Twice the Rayleigh range is defined to be the confocal parameter and is considered to be the length of the beam waist. In this region, the laser beam can be assumed to be a plane wave. For the far-field approximation, the beam waist can be approximated by

$$
w \approx \theta z
$$

where $\theta$ is the half-angle divergence of the beam (in radians) in the far-field limit. 


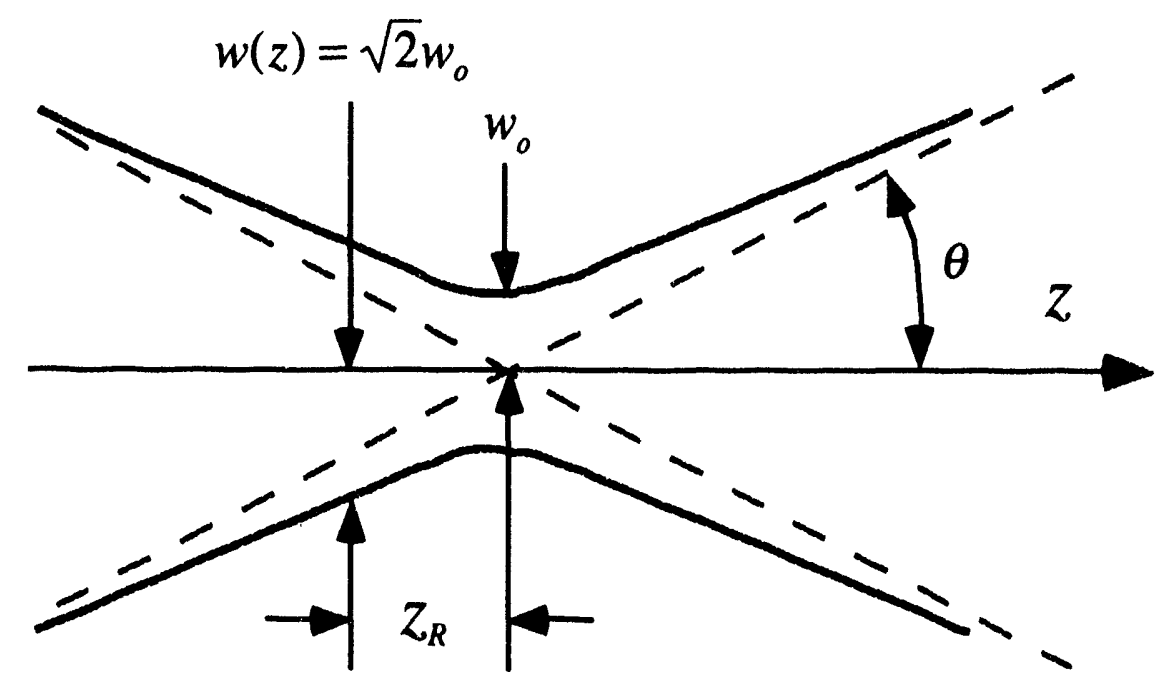

Figure 2.1 Schematic view showing parameters used in defining Gaussian beam propagation. 
To describe the propagation of Gaussian beams through a lens of focal length $f$, Fig. 2.2, the complex image formation equation ${ }^{[11]}$ can be used and is given by

$$
\frac{1}{S+i z_{R}}+\frac{1}{S^{\prime}-i z_{R}^{\prime}}=\frac{1}{f}
$$

where $S^{\prime}$ and $z_{R}^{\prime}$ are the image distance and new Rayleigh range of the newly generated waist, respectively. Rationalizing Eq. (2.4) and then equating the real and imaginary components of the resulting relationship leads to

$$
S^{\prime}=f+\frac{f^{2}(S-f)}{(S-f)^{2}+z_{R}^{2}}
$$

and

$$
z_{R}^{\prime}=\frac{z_{R} f^{2}}{(S-f)^{2}+z_{R}^{2}}
$$

Using these relationships, the position $S^{\prime}$ and Rayleigh range $z_{R}^{\prime}$ for a new beam waist $w_{o}^{\prime}$ can be determined. $w_{o}^{\prime}$ can then be determined using Eq. (2.1) and substituting $z_{R}^{\prime}$ for $z_{R}$.

The relationships described in Eqs. (2.1) - (2.6) are valid for Gaussian beam propagation in the ideal case of diffraction limited beams. However, in reality, ideal diffraction limited beams are not physically realizable due to the finite apertures, scattering, higher mode orders, etc. associated with real laser systems. Thus, to describe real beams accurately, these ideal relationships must be modified to account for deviation from the limiting case. A theory has been developed which allows the real beam parameters to be measured and correlated to the ideal Gaussian case[12,13].

For an ideal Gaussian beam, the waist-divergence product has a fundamental lower limit given by 


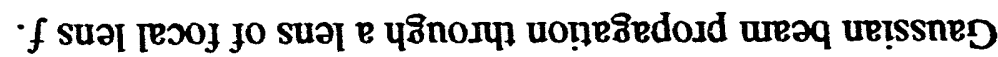

z๐ วגกร!

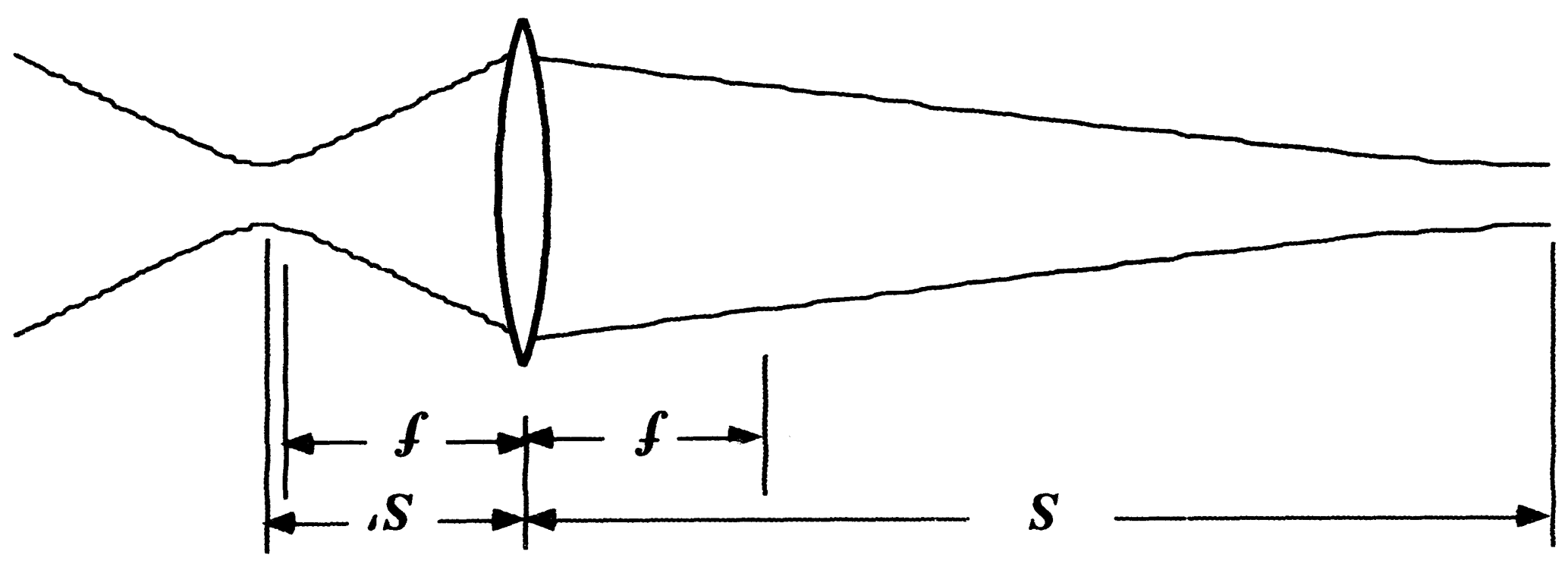




$$
w_{o} \theta=\frac{\lambda}{\pi} .
$$

However, for a real laser beam (Fig. 2.3) the waist-divergence product differs from the ideal case Eq. (2.7) by a factor $M^{2}$, which is referred to as the beam quality factor ${ }^{[12]}$. The beam quality factor is a measure of real laser beam spreading in the far-field with respect to an ideal diffraction limited fundamental transverse mode Gaussian beam. The waist-divergence product for real laser beams is then

$$
W_{0} \Theta=\frac{\lambda}{\pi} M^{2}=w_{0} \theta M^{2},
$$

where $W_{o}$ and $\Theta$ are the real beam waist and half-angle divergence, respectively, and are given by

$$
W_{o}=M w_{o}
$$

and

$$
\Theta=M \theta .
$$

As shown in Figure 2.3, the real beam can be considered to contain an embedded Gaussian beam. As described in Eqs. (2.9) - (2.10), the real beam divergence and waist are each $\boldsymbol{M}$-times the value of this embedded Gaussian beam and the real beam radius varies by a factor of M-times the expected value for the embedded Gaussian beam at any position along the beam propagation axis. For both the real beam and the embedded Gaussian beam, the Rayleigh ranges are equal and defined as

$$
z_{R}=\frac{W_{o}}{\Theta}=\frac{W_{o}}{\theta} .
$$

The relationship given in Eq. (2.11) can be used to determine the embedded Gaussian beam parameters contained within the real beam. Once the embedded Gaussian beam's 


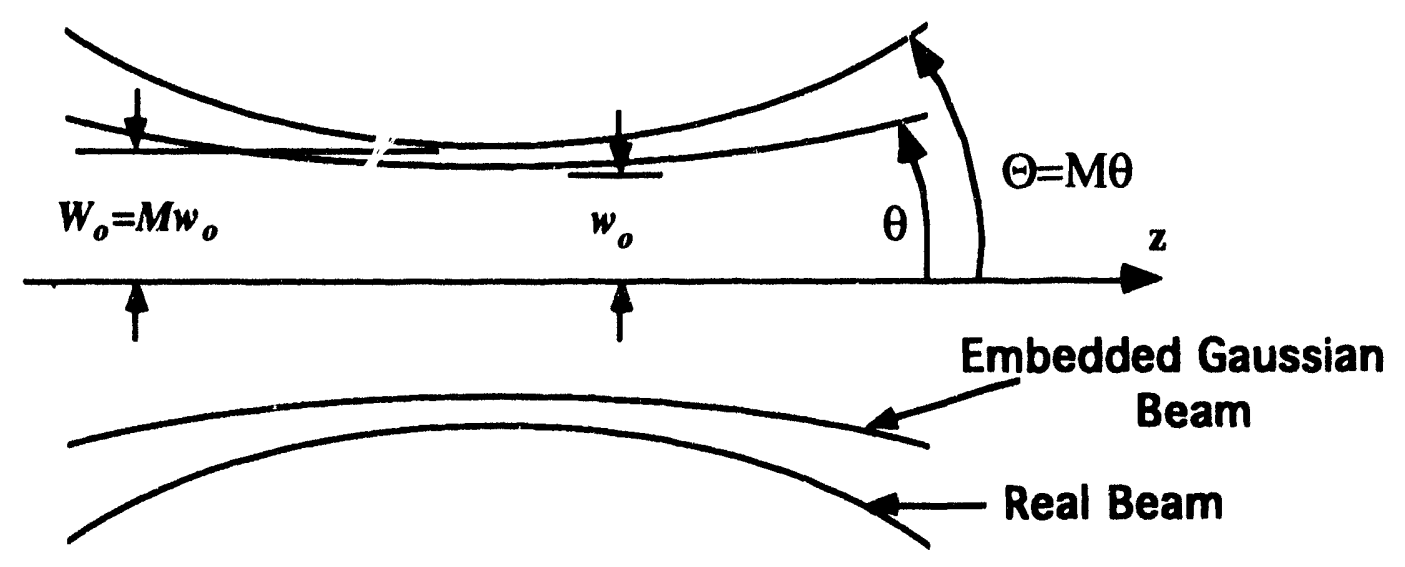

Figure 2.3 Schematic representation of real laser beam with the proposed embedded Gaussian beam. 
parameters are determined, they can be used for optical system design purposes [12]. In such an application, the embedded Gaussian veam is propagated through an optical system using Eqs. (2.4) - (2.6) or ABCD matrices. After propagation through the optical system, the actual (or real) beam parameters can then be determined by use of Eqs. (2.9) (2.10). When mode-matching the real beam to various optical systems, it is important to use the embedded Gaussian beam parameters since this beam contains the correct wavefront radius of curvature information. Also, these relationships are valid for higher mode order beams; however, the statistical distribution of the beam energy profiles needs to be considered in these cases since measuring the beam at the $1 / \mathrm{e}^{2}$ times the peak intensity point can give ambiguous results if the intensity distribution is not Gaussian[12]. This formalism allows higher order mode beam propagation to be described without having to deal with the complications of Laguerre-Gaussian or Hermite-Gaussian field distributions.

\subsection{Fabry-Perot Confocal Cavity}

The Fabry-Perot confocal cavity is a symmetrical cavity and consists, in its simplest form, of two spherical mirrors with identical radii of curvature, (see Fig. 2.4). The mirrors are positioned such that the foci of both mirrors coincide in the center of the cavity; or equivalently, the center of curvature of each mirror falls on the surface of the opposite mirror. For the confocal cavity, the beam waist, located at the center of the cavity, is located one Rayleigh range away from each mirror. Thus, the waist for a confocal cavity is defined by

$$
w_{o}=\sqrt{\frac{R \lambda}{2 \pi}}
$$

where $\mathbf{R}$ represents the radius of curvature of the mirrors. Since the beam waist is located at one Rayleigh range from the mirror, then the beam radius at the mirror is $\sqrt{2} w_{o}$. 


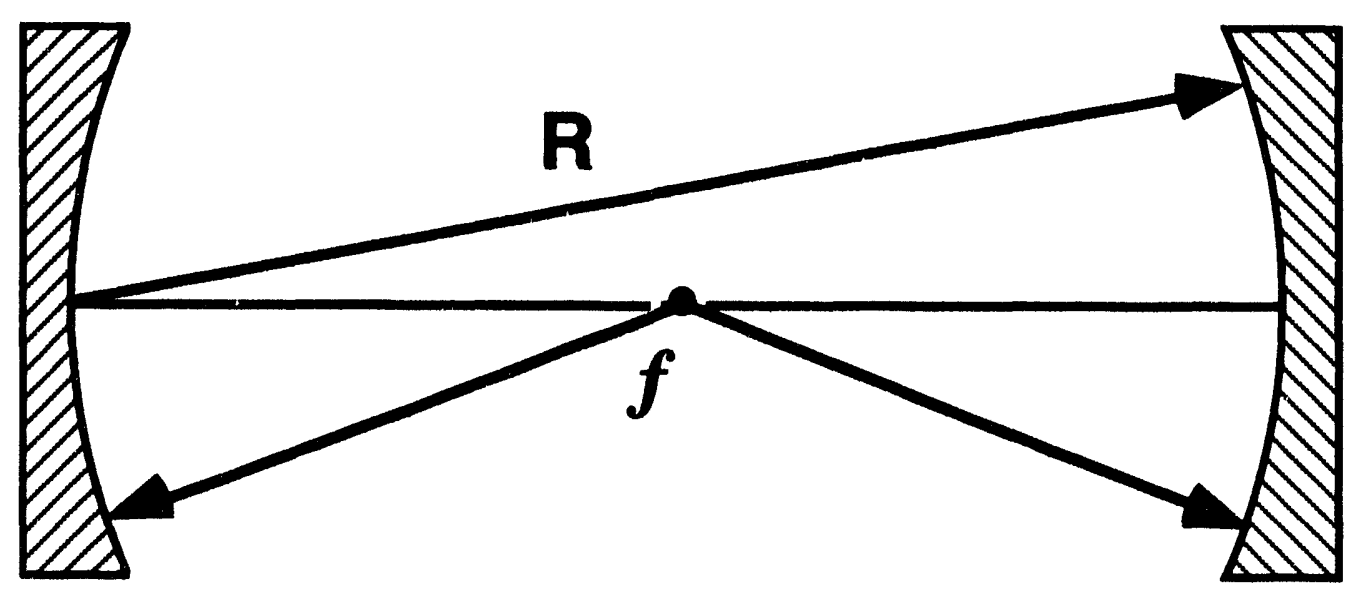

Figure 2.4 Fabry-Perot confocal cavity configuration showing the mirror spacing to be twice the mirror focal length $f$ or, equivalently, at the radius of curvature of the mirrors. 
One advantage of using a confocal cavity is that the cavity is relatively insensitive to misalignment and a tilt in either mirror will only result in displacement of the optical axis of the resonator[14]. A second advantage of the confocal cavity is that all of the cavity transverse modes are degenerate. The even-symmetry transverse cavity modes, i.e. $\mathrm{TEM}_{00}, \mathrm{TEM}_{02}, \mathrm{TEM}_{20}$, etc., are degenerate to positions corresponding to the axial mode spacing given by

$$
\Delta f_{a x}=\frac{c}{2 R}
$$

Thus, every time the cavity length changes by the spacing represented by Eq. (2.13), a transmission peak will occur which is indicative of excitation of the even-symmetry transverse cavity modes. The odd-symmetry transverse modes, i.e. TEM $10, \mathrm{TEM}_{01}$, etc., are also degenerate; however, the odd-symmetry transverse cavity modes degenerate to axial positions which are exactly halfway between the axial modes due to the evensymmetry transverse mode excitation (see Fig. 2.5). So, if many of the cavity transverse modes are excited and the cavity length is changed by the spacing given in Eq. (2.13), then the cavity transmission will have two peaks which represent excitation of both the even- and odd-symmetry transverse cavity modes. This is an important feature of the confocal cavity for spectral analysis of lasers and other optical sources. However, this feature also limits the cavity mode spacing to $c / 4 R$ for a poorly mode-matched cavity compared with a spacing of $c / 2 R$ for a well mode-matched cavity, where $c$ is the speed of light in the cavity.

The power coupled into each of the cavity transverse modes can be determined from the cavity transmission characteristics. Assuming that all of the incident beam power is coupled into the cavity, then the sum of the areas under each of the transmission peaks will give the area that corresponds to the total beam power. Taking the ratio of the area of each of the individual transmission peaks to the total area will then 


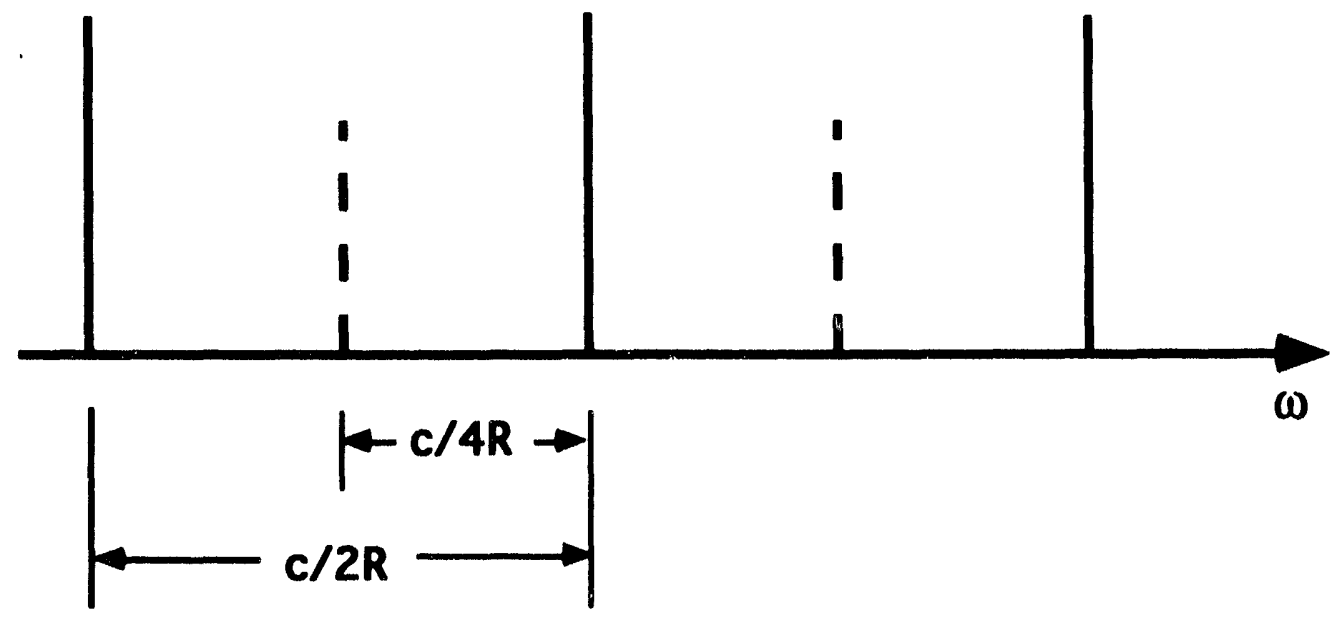

Figure 2.5 Longitudinal mode frequencies for a confocal cavity. Note that the even symmetry transverse modes degenerate to positions indicated by $c / 2 R$ while the odd symmetry transverse modes degenerate to positions between these modes indicated by $\mathrm{c} / 4 \mathrm{R}$. 
give the percentage of beam power coupled into each of the cavity modes. Thus, even for the confocal carity with only the two degenerate axial modes, optimum power coupling into the cavity requires that only one of the transverse cavity modes be excited. Thus, the incident beam parameters must be matched to cavity parameters.

When a single transverse mode of the confocal cavity is excited and the cavity length is varied, then the transmission through the cavity, (see Fig. 2.6) can be used to determine the longitudinal mode structure of the laser as well as the laser linewidth. The distance between similar peaks in the transmission is the free spectral range of the scanning cavity which is given by

$$
\Delta v=\frac{c}{2 R}
$$

which is equal to the cavity axial mode spacing given in Eq. (2.13). A second parameter $\Delta v_{1 / 2}$ defines the cavity resonance peak full-width at half-maximum (FWHM) bandwidth and is given by

$$
\Delta v_{1 / 2}=\frac{c}{2 R F}
$$

where $\mathrm{F}$, the cavity finesse, is a function of the mirror reflectivity, $R_{O}$. The finesse is given by

$$
F \equiv \frac{\pi \sqrt{R_{o}}}{1-R_{o}}=\frac{\Delta v}{\Delta v_{1 / 2}}
$$

It should be noted, however, that the relationships described in Eqs. (2.15) and (2.16) are for an idealized case where the cavity losses are zero which is never the case in reality. The relationship in Eq. (2.16) also assumes that the reflectivities of the cavity input and output mirrors are equal. Thus, for real cavities where losses exist and the mirror reflectivities can vary, a relationship must be used which accounts for these variations. A relationship has been developed which will account for these variations and uses what is known as delta notation ${ }^{[14]}$. 
For a mirror with a power reflectivity $R_{i}$, the mirror reflectivity can be defined in terms of a mirror coupling coefficient $\delta_{i}$ as

$$
R_{i} \equiv e^{-\delta_{i}}
$$

Solution of Eq. (2.17) in terms of the mirror coupling coefficient leads to

$$
\delta_{i}=\ln \left(\frac{1}{R_{i}}\right)=2 \ln \left(\frac{1}{r_{i}}\right)
$$

where $r_{i}$ is the reflection coefficient of the mirror. Then for a standing wave cavity, using the delta notation, a cavity loss parameter $\delta_{c}$ can be defined. $\delta_{c}$ accounts for the losses due to absorption and scattering within the cavity as well as transmission through the mirrors and is given by

$$
\delta_{c}=\delta_{o}+\ln \left(\frac{1}{R_{1} R_{2}}\right)
$$

where $\delta_{o}$ is the round trip linear loss parameter which accounts for scattering and absorption losses. Using this formalism, the cavity finesse is then given by

$$
F \approx \frac{2 \pi}{\delta_{c}}
$$

Then, from Eq. (2.20), it can be seen that the cavity finesse will be reduced as the losses are increased. Thus, measuring the finesse will provide a method for determining the linear losses within the cavity. It should also be noted that the FWHM bandwidth of the cavity resonance peaks will increase with increased cavity losses.

\subsection{Mode-matching}

Although the transverse modes of a confocal cavity will degenerate to two sets of axial modes over one cavity free spectral range, in many circumstances it is desirable to couple 


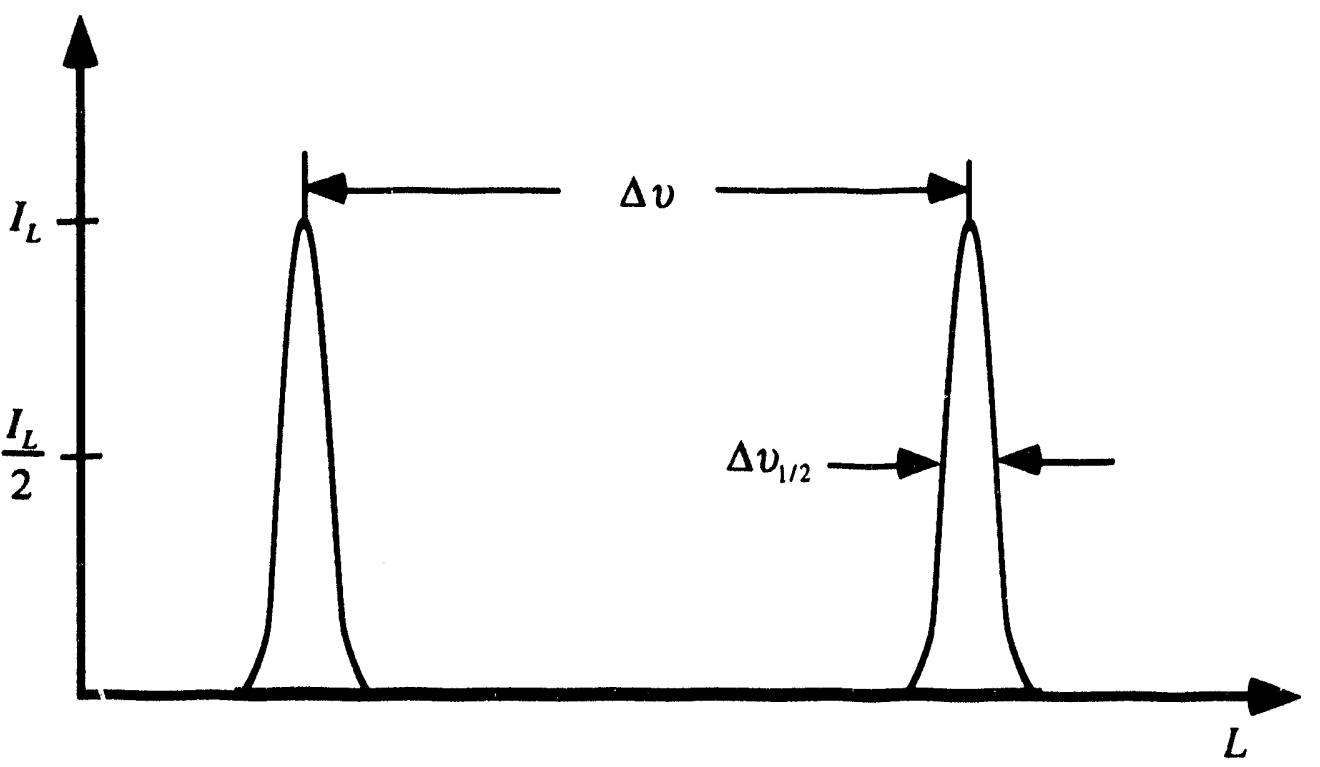

Figure 2.6 Single transverse cavity mode excitation transmission characteristic as a function of cavity length $L$. 
all of the laser power into a single transverse cavity mode, for high power coupling. In this case, it is necessary to match the input beam parameters carefully to the cavity parameters. When mode-matching is performed correctly, the input laser power will be coupled into the cavity axial mode corresponding to excitation of the even-symmetry transverse cavity modes and the transmission peak due to the odd-symmetry transverse modes will disappear. The theory for mode-matching to a spherical mirror resonator has been previously developed $[15,16]$.

In the simplest case ${ }^{[15]}$ (see Fig. 2.7), mode-matching consists of transformation of a beam with a known waist $w_{1}$ to produce a beam with a desired waist $w_{2}$ using a single element lens. For mode-matching, the lens focal length $f$ must b: greater than a characteristic length $f_{o}$ given hy

$$
f_{o}=\pi\left(\frac{w_{1} w_{2}}{\lambda}\right)
$$

After selecting a lens that satisfies Eq. (2.21), the position of the lens with respect to the original beam waist location $d_{1}$ and the transformed beam waist location $d_{2}$ can be determined using

$$
d_{1}=f\left(1 \pm \frac{w_{1}}{w_{2}} \sqrt{1-\frac{f_{0}^{2}}{f^{2}}}\right)
$$

and

$$
d_{2}=f\left(1 \pm \frac{w_{2}}{w_{1}} \sqrt{1-\frac{f_{0}^{2}}{f^{2}}}\right)
$$

The same sign must be chosen in Eqs. (2.22) and (2.23) to obtain a correct solution.

This theory can then be extended to mode-matching of a laser beam with known beam parameters to a cavity also with known parameters (see Fig. 2.8). To mode-1.1atch 


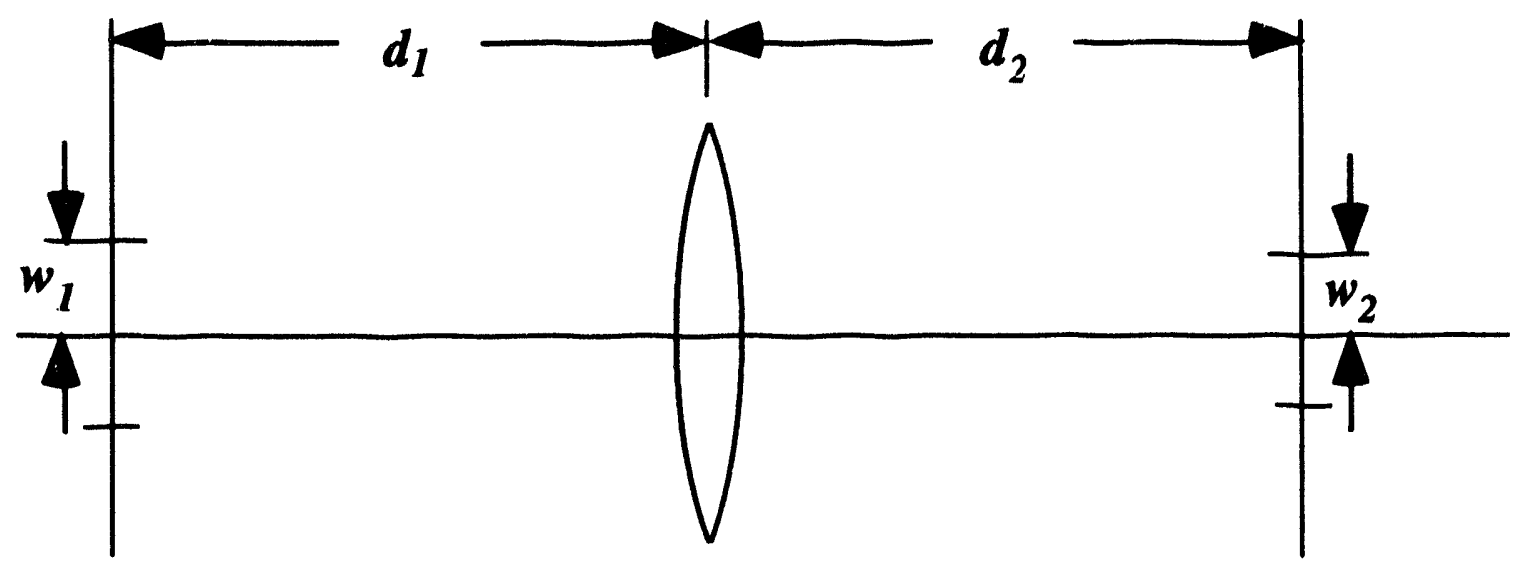

Figure 2.7 Schematic representation of geometry used to mode-match a beam of waist $w_{1}$ to a beam of waist $w_{2}$ using a single element lens.

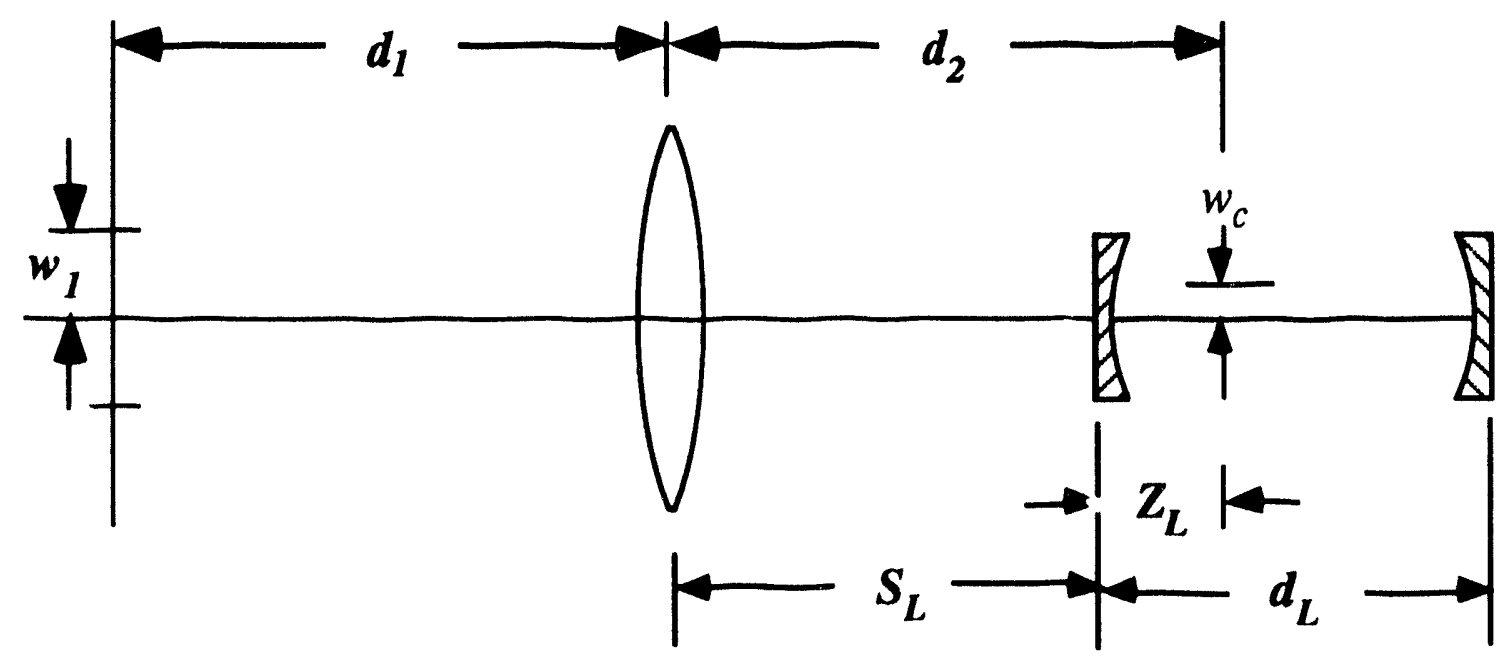

Figure 2.8 Configuration used in mode-matching a beam of a known waist $w_{1}$ to an apparent waist $w_{c}$ within a cavity. 
precisely to the cavity, an apparent beam waist $w_{c}$ within the cavity must first be obtained[16]. This apparint beam waist accounts for the negative lens effect of the cavity input mirror and is described by[16]

$$
w_{c}^{2}=w_{L}^{2} \frac{2\left(R / d_{L}\right)-1}{2\left(R / d_{L}\right)+n^{2}-1}
$$

where $w_{L}$ is the beam radius at the inside of the cavity mirror surface, $d_{L}$ is the length to the outside of the mirrors and $n$ is the index of refraction of the mirror substrate. It is this apparent waist which is used in mode-matching to the cavity. The location of the apparent waist with respect to the input mirror outer surface is given by[16]

$$
z_{L}=\frac{n R}{2\left(R_{o} / d_{L}\right)+n^{2}-1}
$$

The apparent waist value obtained in Eq. (2.24) can be substituted into Eqs. (2.21), (2.22) and (2.23) to obtain the values required for a lens focal length and its location with respect to the laser beam waist and the cavity to optimize mode-matching.

\subsection{Second Harmonic Generation}

When light is transmitted through an optical material, the time-varying electric field associated with the light gives rise to a polarization field within the material[17]. For conventional (or linear) optics, the relationship between the polarization and the electric field is given by

$$
\mathbf{P}(t)=\varepsilon \chi^{(1)} \mathbf{E}(t)
$$

where $\varepsilon$ and $\chi^{(1)}$ are the relative permittivity and the linear susceptibility of the optical material, respectively. For most optical sources, the fields lack sufficient strength to modify the system optical properties significantly; however, with lasers this is not the 
case. The very large electric fields associated with laser beams can act on the optical materials to produce nonlinear effects. For lasers, the polarization must be expanded as

$$
\mathbb{P}(t)=\varepsilon \chi^{(1)} \mathbf{E}(t)+\varepsilon \chi^{(2)} \mathbf{E}^{2}(t)+\varepsilon \chi^{(3)} \mathbf{E}^{3}(t)+\cdots
$$

to include higher order terms to account for the nonlinear susceptibilities of the optical materials. For noncentrosymmetric crystals, i.e. crystals lacking an atomic inversion symmetry, the dominant nonlinear effect is due to the second-order polarization; thus, the higher order terms in Eq. (2.27) can be ignored. It is these types of materials which are used in the second harmonic generation process.

The effect of propagating an intense electric field through a noncentrosymmetric crystal is shown pictorially in Fig. 2.9. As the laser beam propagates through the crystal, a time varying polarization occurs in the material in response to the time-varying electric field associated with the laser beam (see Fig. 2.9 a). The resulting polarization is asymmetrical due to the directionally dependent restoring force associated with the crystal structure. Frequency analysis of the resulting polarization shows that the polarization is composed of three frequency compnnents: the original or fundamental frequency ( see Fig. 2.9 b), the second harmonic frequency (see Fig. $2.9 \mathrm{c}$ ), and a dc component (see Fig. 2.9 d). In these materials, the polarization acts as a source for the second harmonic frequency of the light.

The second harmonic generation process is considered a three wave mixing process, (see Fig. $2.10 \mathrm{a}$ ), where two input waves of frequencies $\omega_{1}$ and $\omega_{2}$ interact with each other to produce an output frequency given by

$$
\omega_{3}=\omega_{1}+\omega_{2} .
$$


(a)

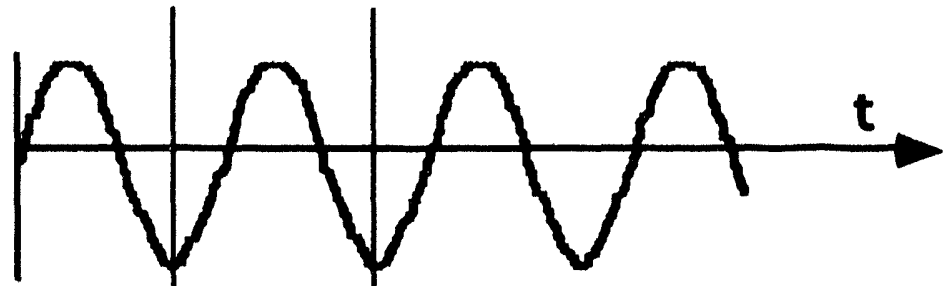

(b)

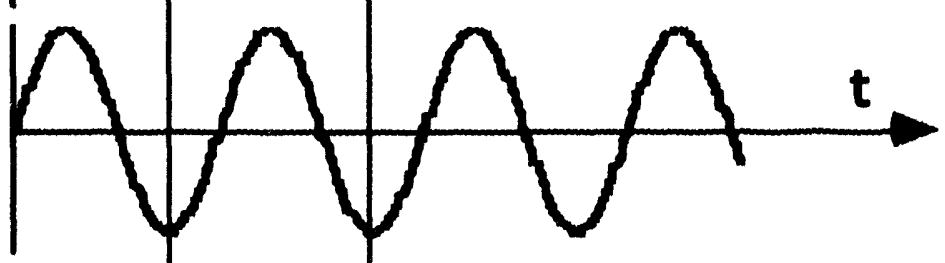

(c)

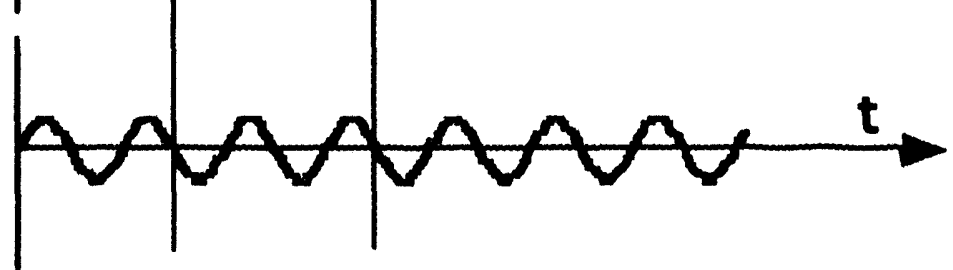

(d)

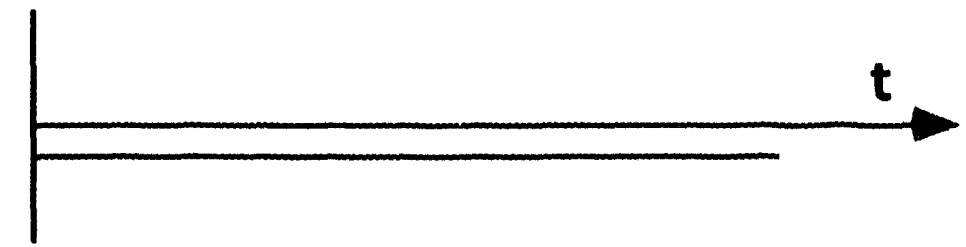

Figure 2.9 Polarization response in noncentrosymmetric crystal due an intense timevarying sinusoidal electric field: (a) asymmetric polarization in NLO crystal, (b) polarization component at fundamental frequency $\omega$, (c) polarization component at SH frequency $2 \omega$, (d) dc component.

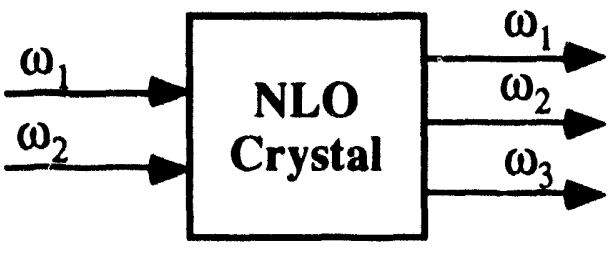

(a)

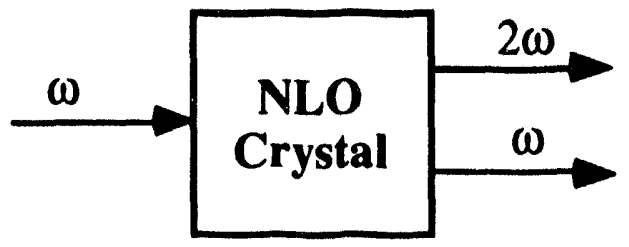

(b)

Figure 2.10 (a) Block diagram for three-wave mixing process or sum frequency generation, (b) SHG is special case of sum frequency generation. 
In the particular case of SHG, it is the interaction of the input beam with itself (Fig. 2.10 b) which leads to generation of light at a frequency exactly twice that of the input frequency, i.e. $\omega_{1}=\omega_{2}=\omega$ and $\omega_{3}=2 \omega$ in (2.23). The parametric interaction which allows transfer of energy from one electric field to another is described by a set of coupled wave equations. Using the first- and second-order susceptibilities associated with the noncentrosymmetric crystal, a nonlinear optical coefficient $d$ can be defined as

$$
d=\frac{m D\left[\chi^{(1)}\right]^{2} \chi^{(2)} \varepsilon_{0}^{3}}{2 N^{2} e^{3}}
$$

where $m$ is the mass of an electron, $D$ is the crystal anharmonic coefficient, $\varepsilon_{o}$ is the permittivity of free space, $N$ is crystal electron density and $e$ is the charge on an electron. Using the nonlinear optical coefficient, the relationship which describes the transfer of energy from light at the fundamental laser frequency to the second harmonic frequency as a function of propagation distance through the NLO medium is described by ${ }^{[3]}$

$$
\frac{d E^{(2 \omega)}}{d z}=-i \omega \sqrt{\frac{\mu}{\varepsilon}} d\left[E^{(\omega)}(z)\right]^{2} e^{i(\Delta k) z},
$$

where $\mu$ is the crystal permeability, $z$ is the propagation distance and

$$
\Delta k \equiv k^{(2 \omega)}-2 k^{(\omega)}
$$

Here $k^{(2 \omega)}$ and $k^{(\omega)}$ are the propagation wavevectors for the SH and fundamental waves respectively. When the single pass conversion of the wave at the fundamental frequency to the SH frequency is much less than unity, depletion of the pump source is considered to be negligible. For this condition, $E^{(\omega)}(z)$ can be assumed to be constant and thus independent of $z$. Also, when there is no input to the NLO crystal at the SH frequency, $E^{(2 \omega)}=0$. Then, subject to these constraints, solution of Eq. (2.29), in terms of intensity, leads to

$$
P_{2 \omega}=2\left(\frac{\mu}{\varepsilon_{o}}\right)^{\frac{3}{2}} \frac{\omega^{2} d^{2} l^{2}}{n^{3}} \sin c^{2}\left(\frac{\Delta k l}{2}\right) \frac{P_{\omega}^{2}}{A}
$$


where $l$ is the crystal length, $n$ is the index of refraction of the crystal at the light fundamental wavelength and $A$ is the area of the beam entering the crystal. It should be noted here that the conversion efficiency is defined as the ratio of the $\mathrm{SH}$ beam output power to the fundamental beam input power. In the relationship described in Eq. (2.32), the conversion efficiency $\eta$, given by

$$
\eta=\frac{P_{2 \omega}}{P_{\omega}}
$$

is proportional to the input beam intensity, $P_{\omega} / A$.

Although lasers possess the large electric fields necessary to produce efficient SHG, a second criterion must also be met. For the SH light to build as it propagates through the NLO crystal, then the two beams at the different frequencies, $\omega$ and $2 \omega$, must propagate through the crystal with the same velocity. When this condition is satisfied, the optical fields are said to be phase-matched. Then, in Eq. (2.31), $\Delta k=0$ and the SH power will increase as the optical field propagates through the NLO crystal. If this condition is not satisfied, the SH light produced at one position along the crystal optical axis will interfere destructively with SH light produced at a second position along the crystal optical axis. Then for a long crystal (with respect to optical wavelength), destructive interference will significantly impede the buildup of SH power within the NLO crystal. The destructive interference effect can be minimized by limiting the crystal length; however, since the conversion is proportional to the square of the crystal length, this is not a desirable option.

For isotropic crystals this condition might be satisfied if (a) an artificial structure such as a waveguide or grating provides dispersion or (b) the material has anomalous dispersion. However, an anisotropic crystal possesses birefringence which can be exploited to satisfy this condition. The birefringent crystal has a polarization sensitive index of refraction so the crystal can be oriented such that 


$$
n(\omega)=n(2 \omega)
$$

thus satisfying the phase matching condition, $\Delta k=0$. It should be noted that since a laser beam has a finite size and divergence associated with it, then this phase-matching condition can never be satisfied completely over the laser beam cross-section.

As mentioned earlier, the conversion efficiency of the SHG process is proportional to the laser beam intensity within the NLO crystal. Thus for low power laser beams, the conversion efficiency of the SHG can be enhanced by focusing the beam through the crystal. The relationship describing second harmonic generation using focused Gaussian beams is given by [3]

$$
P_{2 \omega}=2\left(\frac{\mu}{\varepsilon_{o}}\right)^{\frac{3}{2}} \frac{\omega^{2} d^{2} l^{2}}{n^{3}}\left(\frac{P_{\omega}^{2}}{\pi w_{o}^{2}}\right) \operatorname{sinc}^{2}\left(\frac{\Delta k l}{2}\right)
$$

The conversion efficiency for a focused Gaussian beam, as defined by Eq. (2.33), is proportional to the focused fundamental beam intensity, $P_{\omega} / \pi w_{o}^{2}$. This result is consistent with the result for the nonfocused SHG.

\subsection{Pumped-cavity Second Harmonic Generation}

For low intensity lasers, the problem of how to increase the SH conversion efficiency remains. Examination of Eq. (2.35) indicates that several parameters can be used for SHG optimization: obtain a crystal with a high nonlinear coefficient $d$, increase the NLO crystal length $l$, decrease the beam radius $w_{o}$ and increase the power of the fundamental beam. If these parameters have been optimized, inefficient conversion is still being obtained, then alternative methods must be investigated to improve the SH conversion process. One method used to increase fundamental wavelength intensity available for SH conversion process is pumped-cavity second harmonic generation. 
The pumped-cavity SHG process exploits the circulating power enhancement provided by a resonant optical cavity to effectively increase the beam intensity available for conversion at the fundamental wavelength $[7,18,19]$. In a resonant optical cavity, the circulating power can be many times higher than that of the incident beam and is a function of the reflectivity of the cavity mirrors as well as the cavity losses. By placing the NLO crystal within such a cavity (see Fig. 2.11) the effective intensity available for SHG can be increased dramatically.

For the cavity shown in figure 2.11 , with lossless mirrors, then

$$
R_{1}+T_{1}=R_{2}+T_{2}=1
$$

where $R_{1}, T_{1}, R_{2}$ and $T_{2}$ are the power reflection and transmission coefficients for the input and output mirrors, respectively. Let $T$ be the transmission coefficient for the material within the cavity when no nonlinear conversion is occurring and $T_{S H}$ be the fundamental wavelength transmission through the NLO crystal which accounts for the conversion to the second harmonic and is given by

$$
T_{S H}=\left(1-\gamma_{S H} P_{c}\right)
$$

where $\gamma_{S H}$ is the nonlinear conversion factor and $P_{c}$ is the circulating power within the cavity. The nonlinear conversion factor $\gamma_{S H}$ accounts for laser beam focusing within the NLO crystal and is given by

$$
\gamma_{S H}=\frac{2 \omega^{2} d^{2} k_{\omega}}{\pi n^{3} \varepsilon_{o}^{2} c^{3}} \ln (B, \xi)
$$

where $k_{\omega}$ is the fundamental beam propagation factor within the NLO crystal, and $h(B, \xi)$ is the Boyd and Kleinman focusing factor ${ }^{[18]}$. Then letting the cavity reflectance parameter be 


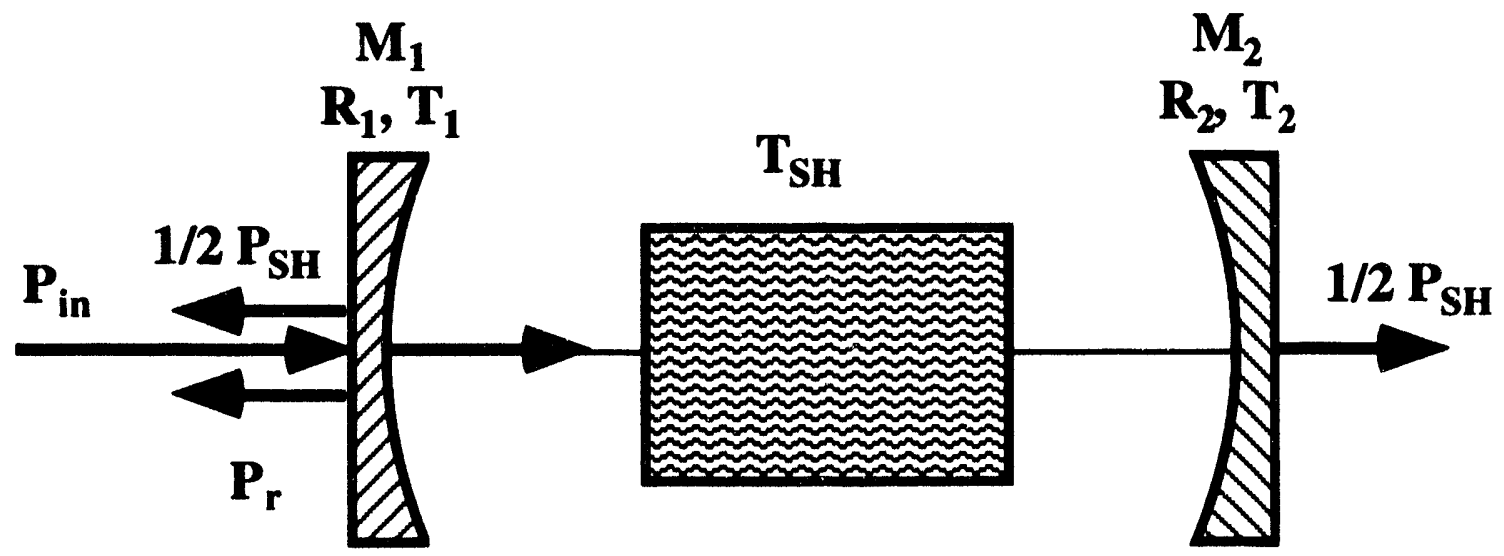

Figure 2.11 Schematic representation of pumped-cavity second harmonic generation configuration 


$$
R_{m}=T^{2} T_{S H}^{2} R_{2}
$$

leads to the circulating power enhancement within the cavity given by

$$
\frac{P_{c}}{P_{\text {in }}}=\frac{T_{1}}{\left(1-\sqrt{R_{1} R_{m}}\right)^{2}} .
$$

The ratio of the reflected power to the power incident onto the cavity is given by

$$
\frac{P_{r}}{P_{\text {in }}}=\frac{\left(\sqrt{R_{1}}-\sqrt{R_{m}}\right)^{2}}{\left(1-\sqrt{R_{1} R_{m}}\right)^{2}} .
$$

To maximize the coupling of the laser power into the cavity, the cavity reflectance must be minimized. From Eq. (2.41) it can be seen that this condition will occur when $R_{l}=$ $R_{m}$, which is referred to as the impedance match condition $[7]$. From this formalism, it can be shown that the SHG power for a standing wave cavity is given by

$$
P_{2 \omega}=2 \gamma_{S H} P_{c}^{2}
$$

and then that the conversion efficiency is

$$
\eta_{S H}=\gamma_{S H} P_{c}
$$

The results from this theory indicate that a large enhancement in circulating power within the cavity can lead to a very high conversion efficiency. It is important in the case of pumped-cavity SHG to use high quality optical materials within the cavity since the linear losses will limit the circulating intensity thus reducing the intensity available for SHG.

It is important to note that the non-depleted source SHG approximation has been used in development of this model. Although the overall conversion efficiency from the input 
fundamental beam to the output SH beam can be large, the conversion efficiency of the circulating intensity within the cavity to the output $\mathrm{SH}$ beam intensity is small. For the low conversion efficiency within the cavity the single-pass depletion of the circulating fundamental beam is small, thus the non-depleted source approximation is still approximately valid.

\section{$2.6 \quad$ Frequency-Locking}

To maintain optimum output of the SH light, the cavity length must satisfy the condition that the round-trip distance in the cavity $L$ is

$$
L=m \lambda
$$

where $\mathrm{m}$ is an integer and $\lambda$ is the laser wavelength. As shown in figure (2.6), the transmission characteristic for a high finesse optical cavity exhibits very narrow, steep behavior. This is primarily due to the fact that, for a high finesse cavity, the light contained within the cavity makes many round-trips before escaping through the output mirror. Hence, a small deviation from the condition established in Eq. (2.42) will lead to destructive interference between the input and circulating beams, leading to a significant reduction in the circulating intensity within the cavity.

Several factors can influence the output wavelength from a laser: thermal drift, electronic noise, etc. These factors can cause the laser wavelength to vary randomly with time and thus change the laser's ability to satisfy the condition established in Eq. (2.42) for prolonged periods of time. In addition, even for a relatively short cavity, the integer value $m$ in Eq. (2.42) can have a value of several thousand. Due to this relatively long optical path length, sinall variations in the cavity index of refraction or mechanical vibrations can lead to random variations in the cavity length of sufficient magnitude to inhibit the ability of even the most stable laser source to satisfy the condition established 
in Eq. (2.42) for very long. In general, fluctuations due to the laser and due to the cavity also vary randomly with respect to each other. Thus the best way to compensate for these random fluctuations is to use a method of feedback control to maintain the resonant condition between the laser and the cavity. For optimum results, it is best to identify the system component which has the highest frequency variations and then control this component.

For diode lasers, the output wavelength is dependent on thermal drift of the device, the input current of the power supply, and some random variations due to electrical noise ${ }^{[20]}$. Although the fluctuations due to thermal drift can be slow, on the order of seconds, the variations due to the electrical noise have a much higher frequency that can vary much faster than fluctations in the cavity length. Thus, for diode lasers, it is best to use feedback control to lock the laser wavelength to the cavity resonance peak. This is often referred to as frequency-locking.

A dithering technique can be used to provide a control signal for frequency-locking of the laser to the cavity resonance peak[21]. Figure 2.12 illustrates how this technique can be used to produce an error signal for feedback control. A small sinusoidal modulation is used to, in effect, vary the cavity length around the resonant condition. This effect can be induced by several methods: physically modulating the cavity length, modulation of the laser current, etc. However, for illustration purposes, it will be assumed that it is the laser wavelength which is being modulated and that the effect of modulation is being measured by intensity variations in the cavity transmission.

When the cavity is near resonance, position $\{1\}$, modulation of the laser wavelength will give a very small peak-to-peak amplitude ac signal at the detector. However, when the wavelength of the laser shifts with respect to the resonance peak, positions $\{2\}$ and $\{3\}$, 


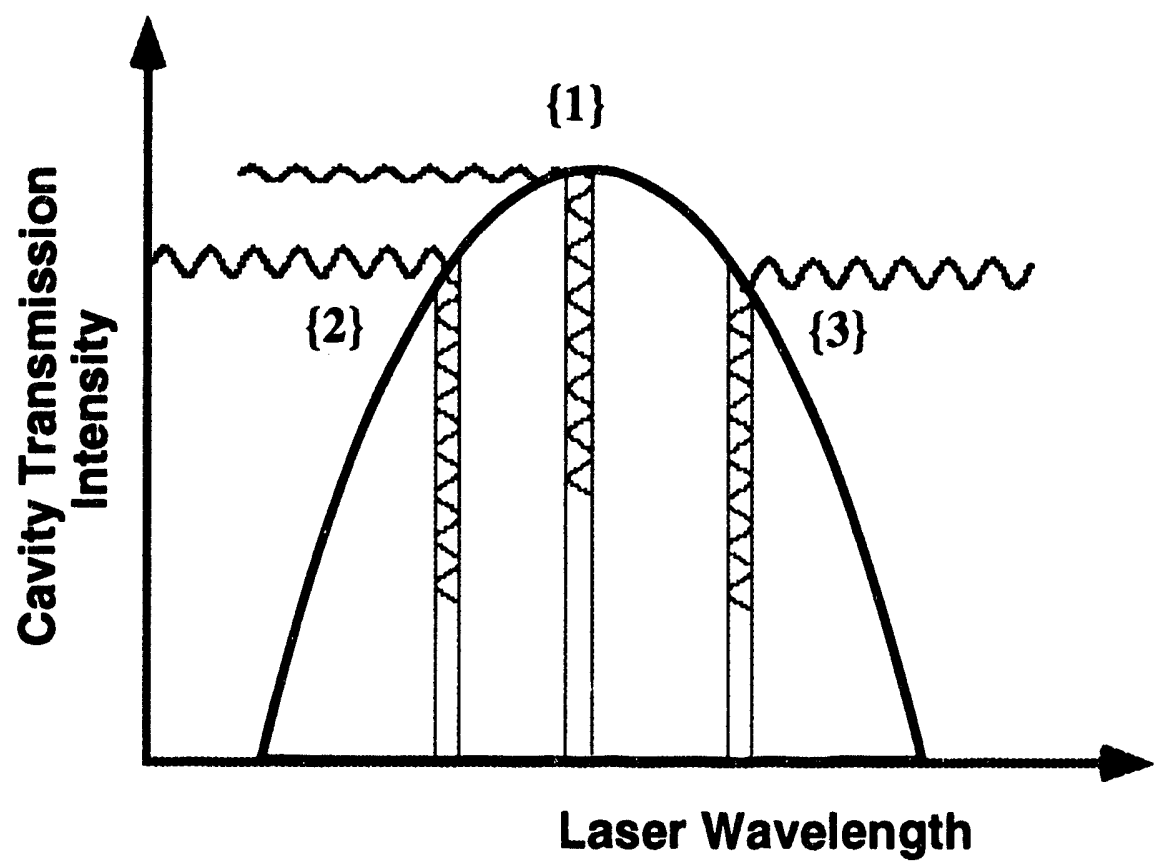

Figure 2.12 Dither technique used frequency-lock the diode laser wavelength to the cavity resonance peak. 
then the same wavelength modulation will produce a much larger peak-to peak amplitude ac signal at the detector. The peak-to-peak amplitude of the detected signal will increase approximately linearly, in a region around the resonant transmission peak, as the laser wavelength moves further away from the resonant condition. The amplitude of this signal is symmetric with respect to the resonant peak, thus the amplitude alone cannot be used as a control signal.

A closer look at the detected signals a' nositions $\{2\}$ and $\{3\}$ provides a solution to the problem posed. The detected signal at position $\{2\}$ increases in intensity as the laser wavelength is increased, thus the two signals are in phase. However, for position $\{3\}$, when the laser wavelength is increased the light intensity at the detector decreases indicating a $\pi$ phase shift between these two signals. Thus, the solution is to use a phase sensitive detector which will provide an error signal that is positive when the modulated and detected signals are in phase and is negative when the modulation and detected signals are out of phase. In addition, the error signal amplitude must be proportional to the peak-to-peak amplitude of the detected signal. The expected signal obtained using this technique is shown in Figure 2.13. For stable feedback control, it is necessary to operate the system within the linear region of this curve, i.e. between points A and B.

\subsection{Summary}

In this section we have presented an overview of the theory used in the development of the pumped-cavity second harmonic generation experiment. The relationships for Gaussian beam propagation in the ideal limiting case were presented. Then relationships were presented for real laser beams which established connections between the real and idealized laser beam and allowed the idealized Gaussian beam propagation theory to be used to predict real beam propagation behaviors. 


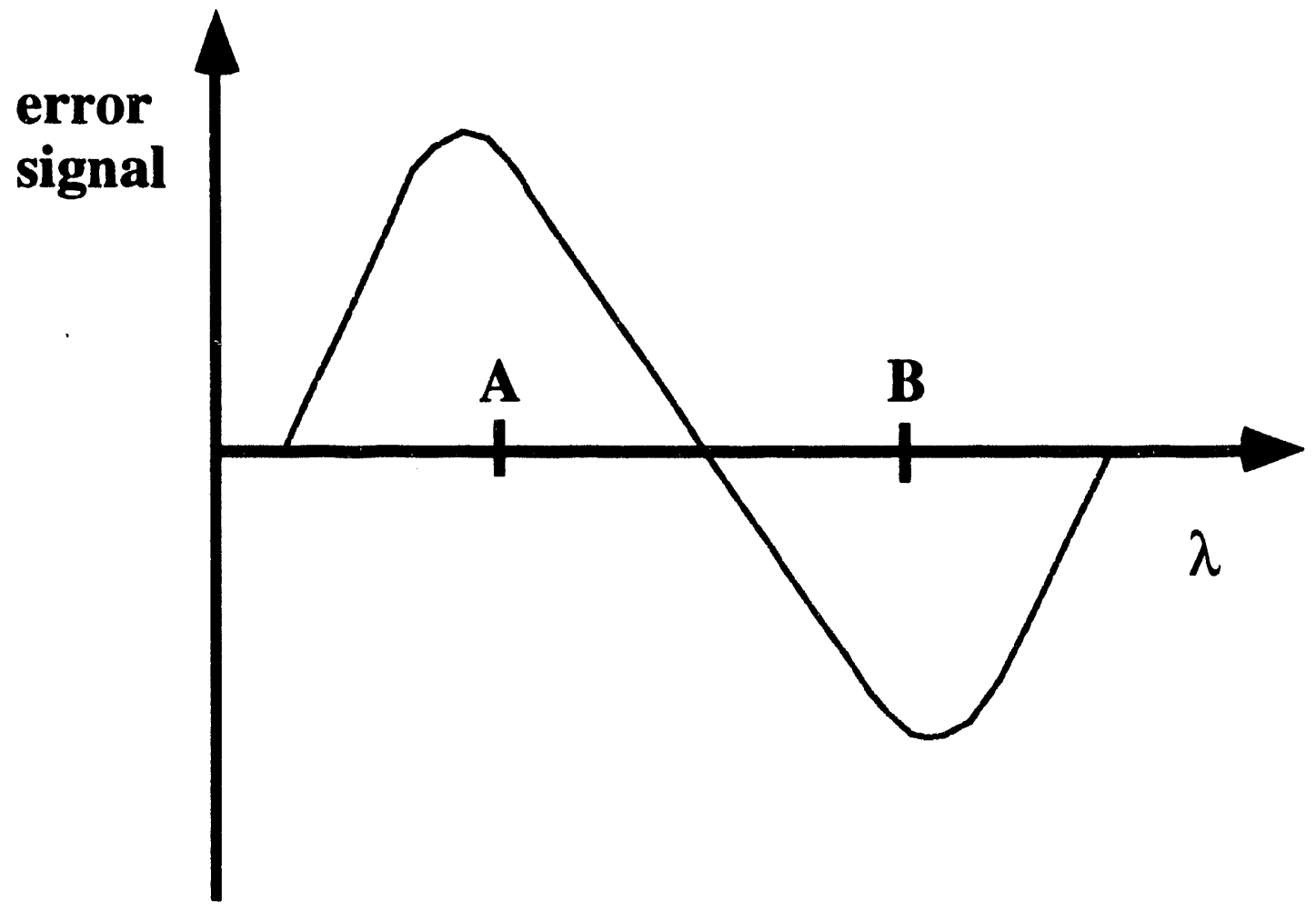

Figure 2.13 The expected error signal as the laser wavelength is scanned through the cavity resonance peak (note: region of stable operation is between points $\mathrm{A}$ and $\mathrm{B})$. 
Next, the characteristic provided by Fabry-Perot confocal cavities were presented. Relationships describing the cavity free spectral range, the cavity resonance peak FWHM bandwidth and the cavity finesse were all given. The dependence of the tinesse on cavity losses was established so that the finesse measurements could be used to determine the losses within a cavity. Then the equations describing mode-matching between a beam with known Gaussian beam parameters to a cavity were given.

An overview of the theory of SHG was provided and the relationships for single-pass SHG presented. These relationships represented the limiting case where a nondepleted source approximation is assumed. This overview of the theory served as an introduction for the theory of pumped-cavity SHG. Relationships were given to describe the pumpedcavity SHG process and the necessary conditions for optimum conversion were established.

Finally, the concept used in creating a frequency-locking system was presented. 


\section{CHAPTER 3}

\section{Second Harmonic Generation Experiments}

\subsection{Laser Beam Manipulation}

The laser used in these experiments was a Spectra Diode Labs model SDL-5422-H1 150 $\mathrm{mW}$ CW single mode GaAlAs laser diode[20]. The diode laser output wavelength was approximately $860 \mathrm{~nm}$ and was chosen to provide room temperature phase-matching to

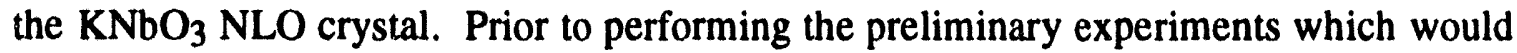
lead to the pumped-cavity second harmonic generation experiment, the output beam from the diode laser needed to be collimated, circularized and then characterized. As shown in Figure 3.1, the raw beam from the diode laser has a divergence angle of $30^{\circ}[20]$ in the plane formed by the optical axis and a vector normal to the diode junction (perpendicular plane). The divergence angle is $10^{\circ}[20]$ in the plane formed by the beam optical axis and a vector parallel to the diode junction (parallel plane). The beam divergence variations between these two planes is due to diffraction from the different sized emitting apertures associated with each of the two planes. Although the beam profile in each plane is Gaussian-like, due to the large variation in divergence in these two planes, the output beam cross-section was elliptical.

The first step in preparing the laser for optimum coupling into the cavity was to collimate the laser output. This was accomplished by placing a collimating lens one focal length away for the beam emitting aperture (see Fig. 3.2). Due to the large beam divergence, a short focal length collimating lens with a working distance of $\approx 3 \mathrm{~mm}$ between the diode output and the lens input surface was used to maintain a small beam diameter. This lens was designed specifically for diode laser collimation and minimizes aberrations introduced to the beam. Using this short focal length lens made positioning the laser with 


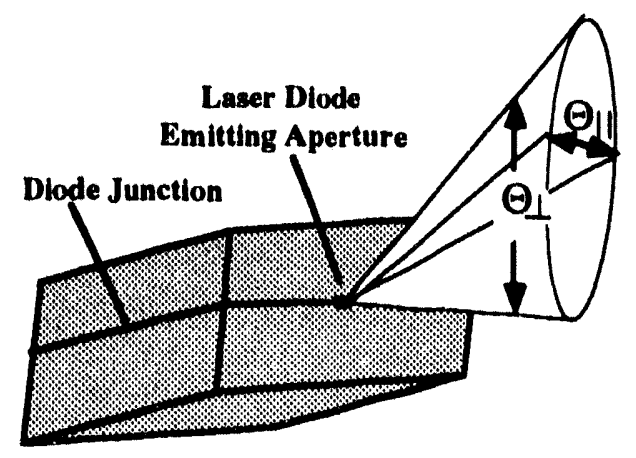

Figure 3.1 Simplified drawing of semiconductor diode laser showing differing divergence angles for the output laser beam.

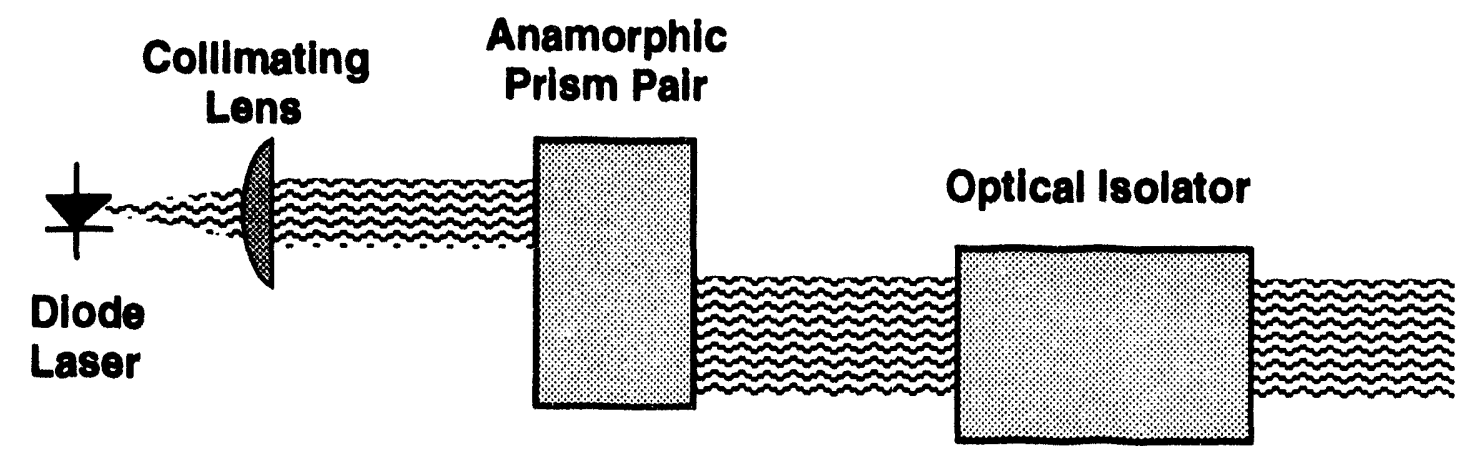

Figure 3.2 Schematic view of optical elements required to prepare the diode laser for the experiments leading up to and including the pumped cavity second harmonic generation experiment. 
respect to the lens very sensitive; thus, the laser was mounted on a micrometer driven stage for precise positioning.

After the beam was collimated, the collimated output was made to be approximately circular by passing the beam through an anamorphic prism pair (see Fig. 3.2) which had an expansion ratio of 3:1. The anamorphic prism pair serves to expand the beam width in the plane of incidence (i.e. the plane defined by the beam propagation vector and the normal to the prism surface) while leaving the beam width in the orthogonal direction unaffected (see Fig. 3.3). Upon passing through the first prism, the direction of propagation of the beam is changed. The second prism is positioned so that the beam is allowed to expand in the plane of incidence until a desired expansion ratio is achieved. The beam is then refracted into the second prism to arrest the beam expansion at the desired ratio and the beam propagation direction is deviated to travel in the direction parallel to the original optical axis. There is, however, a net shift in the beam optical axis with respect to the original optical axis. Meanwhile, light in the plane orthogonal to the plane of incidence is transmitted through the prism pair with its size unaffected.

Finally, to eliminate optical feedback effects, the modified laser beam was passed through a dual Faraday optical isolator which provided $60 \mathrm{~dB}$ of isolation. It was essential to eliminate unwanted optical feedback to the diode laser due to the destabilizing effect that the optical feedback has on these lasers[22].

\subsection{Laser Beam Parameter Measurement}

After collimation, expansion, circularization and isolation of the beam were achieved, then the modified laser beam parameters were measured. The first beam characteristic measured was the linewidth of the laser emission. To measure the laser linewidth, a scanning spherical mirror interferometer was used. The cavity length was scanned by 


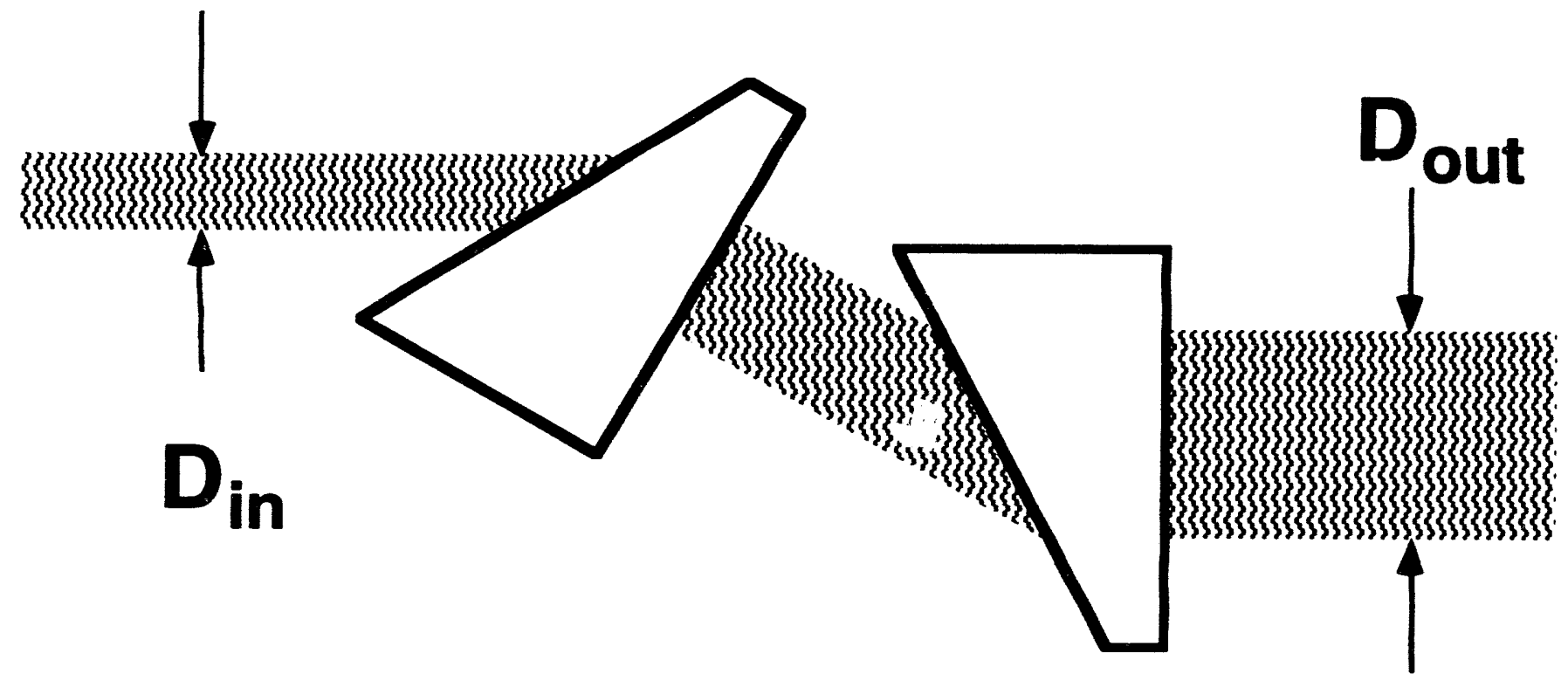

Figure 3.3 Effect of using anamorphic prism pair to increase the laser beam width in a single plane. Note that the beam width in the orthogonal plane in unaffected. 
applying a sawtooth modulation voltage to a piezoelectric transducer which periodically varied the cavity length. The light transmitted through the cavity was then focused onto a photodetector and the electrical signal due to the transmitted light was captured on a digital storage oscilloscope for analysis.

A high finesse, $25 \mathrm{~cm}$ confocal cavity was used to construct a scanuing interferometer to perform laser linewidth measurements. From measurements of the cavity transmission characteristics, the cavity resonance peak FWHM bandwidth and free spectral range were determined. These measured values were used to calibrate the scanning interferometer and determine the linewidth of the laser as a function of laser output power. The measured laser linewidth was compared to the resonance peaks FWHM bandwidth for the various cavities which were to be used in subsequent experiments. It is important for optimum power coupling into the cavity to have a laser linewidth which is much smaller than the FWHM bandwidth of the pumped-cavity resonance peaks. In addition, the ability to lock the laser frequency to the cavity is related to the difference between the laser linewidth and the cavity resonance peak FWHM bandwidth. To improve the laser frequency-locking stability, the bandwidth of the laser should be small with respect to the cavity resonance peak FWHM bandwidth.

To align the $25 \mathrm{~cm}$ cavity and optimize the finesse, it was necessary to use a light source possessing a linewidth less than the cavity resonance peak FWHM bandwidth. Since no sources were available with sufficiently narrow linewidth at the wavelength for which the mirrors were designed, optical feedback was applied to the laser diode to reduce its linewidth ${ }^{[23]}$ (see Fig. 3.4). By applying optical feedback to the laser diode, the laser linewidth was reduced sufficiently to allow the cavity to be optimized. After the cavity was optimized, its transmission characteristics were recorded so that the cavity finesse could be determined from these measurements. The cavity finesse is simply the ratio of 


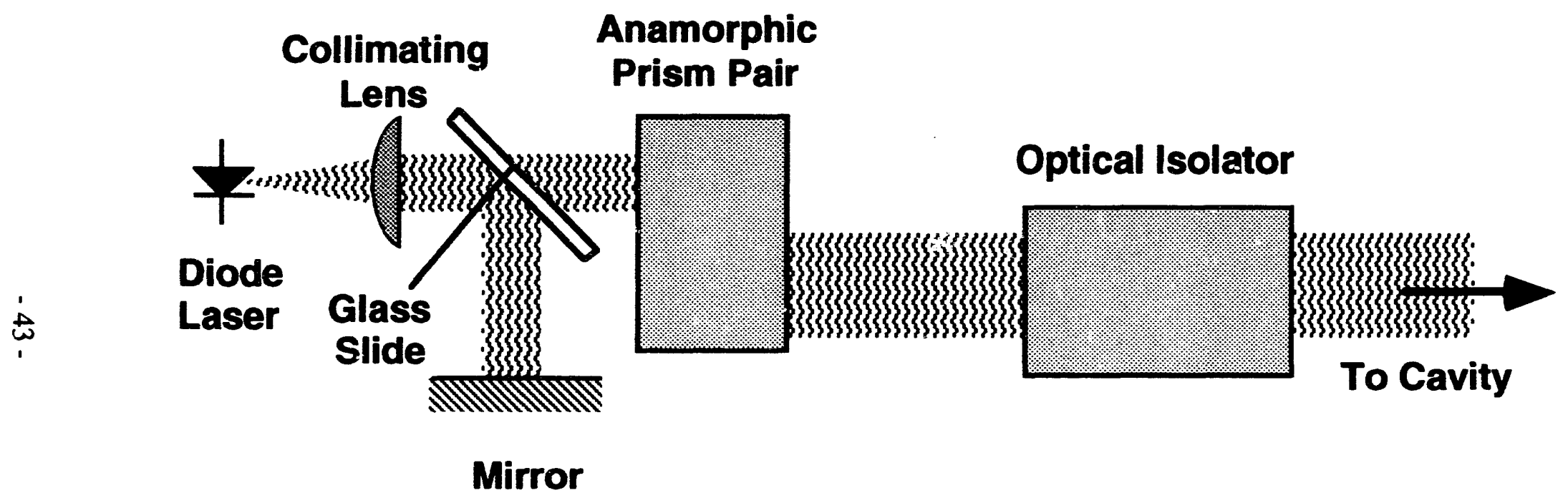

Figure 3.4 Figure showing how optical feedback was introduced to diode laser to reduce the linewidth of the laser emission. 
the free spectral range $\Delta v(t)$ to the minimum cavity resolution $\Delta v_{1 / 2}(t)$ (see figure 3.5), where $\Delta v_{1 / 2}(t)$ is taken as the FWHM point of the cavity transmission peak. The measured finesse was then used with the theoretical free spectral range of the cavity calculated using Eq. (2.14) to determine the FWHM bandwidth of the scanning cavity interferometer resonance peak in terms of frequency.

After the cavity was aligned and its finesse measured, the optical feedback to the laser was removed so that the true laser beam linewidth could be measured. For this measurement, the cavity length was scanned and the cavity transmission characteristics were captured on an oscilloscope. Taking the ratio of the previously measured cavity free spectral range $\Delta v(t)$ to the calculated theoretical free spectral range $\Delta v$ (Eq. (2.14)), a calibration factor was obtained. This calibration factor was used to convert the measured laser linewidth $\Delta v_{1 / 2}(t)$, in terms of time, to a linewidi in terms of frequency. The diode laser linewidth was measured for powers varying from 14 to $140 \mathrm{~mW}$.

Next, the Gaussian beam parameters of the diode laser were measured. To determine the laser's Gaussian beam parameters, a waist was produced using a $250 \mathrm{~mm}$ focal length lens with a $f / \# \approx 40$ to avoid introducing aberration into the beam. The beam diameter was measured in the planes corresponding to the previously defined planes perpendicular and parallel to the diode junction. These measurements were made using a commercially available scanning slit device, Beam Scanner from Merchantek, which has a resolution of $5 \mu \mathrm{m}$ [24]. This device allows the beam to be repetitively scanned while simultaneously measuring the beam waist in two orthogonal directions.

The configuration used for Gaussian beam parameter measurements is shown in Figure 3.6. The beam scanner was mounted to a micrometer driven stage which allowed 100 $\mathrm{mm}$ of adjustment along the beam optical axis. This permitted the beam diameter to be 


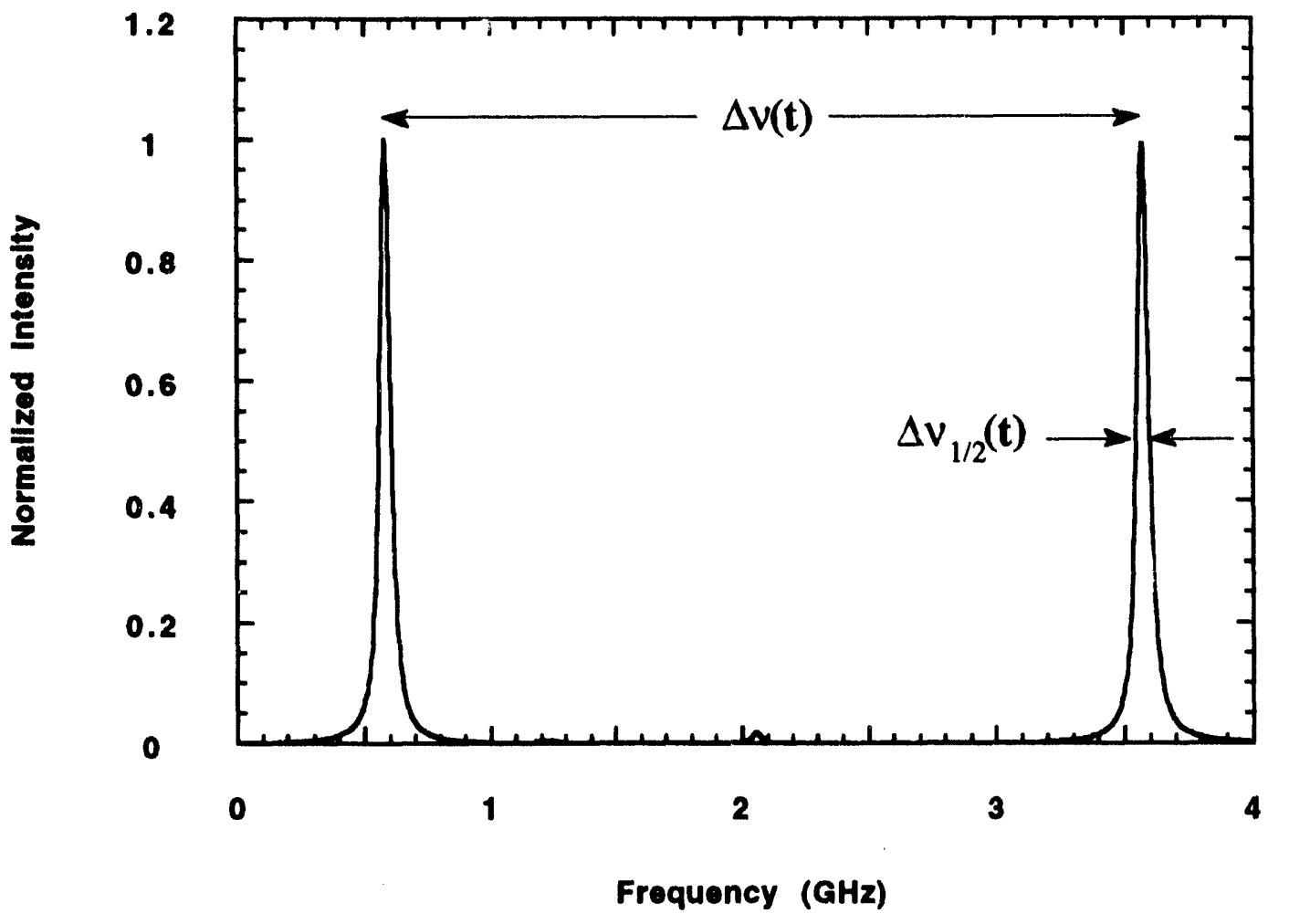

Figure 3.5 Transmission characteristics from the scanning Fabry-Perot interferometer as a function of time.

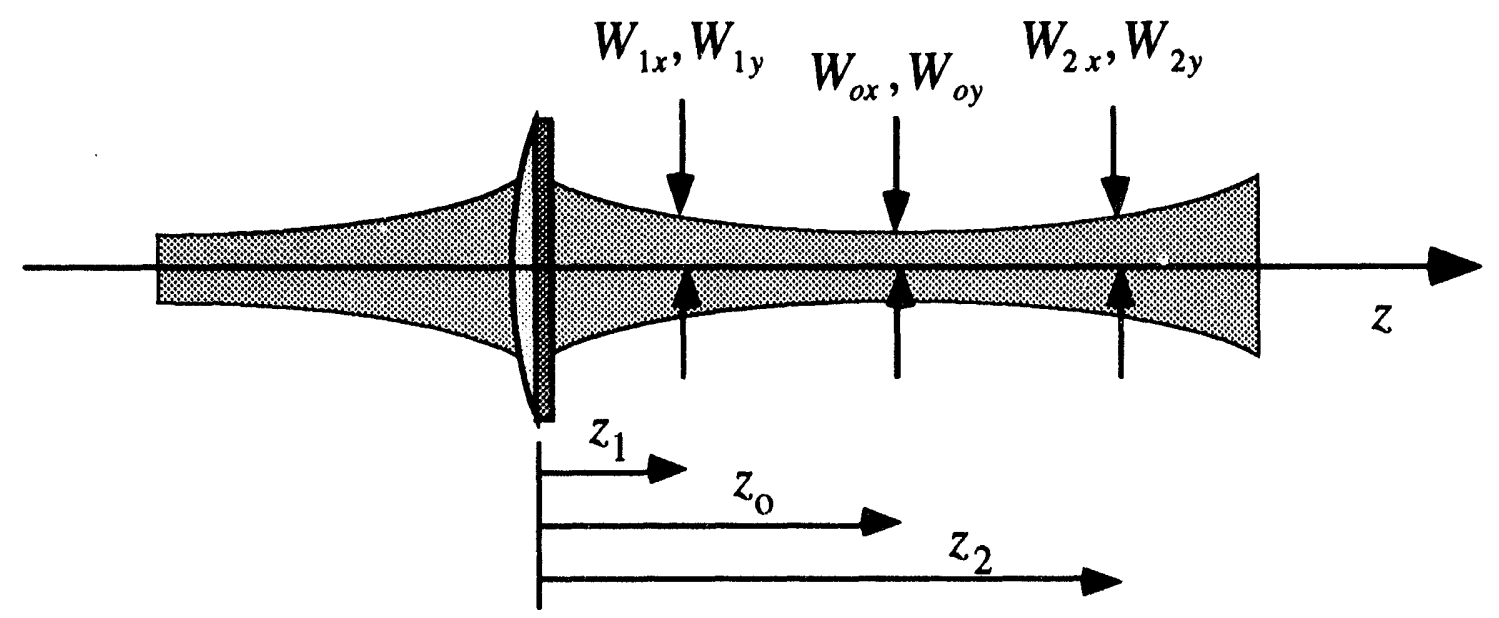

Figure 3.6 Diagram showing scheme used in characterizing real laser beam to determine Gaussian beam parameters. 
measured at precise locations along the optical axis with respect to a predetermined reference point. The initial measurements were taken at a position $z_{1}$ along the optical axis where the beam diameter was several times that of the minimum waist value. The values for beam radii $W_{1 x}$ and $W_{1 y}$, as well as the position with respect to the reference plane, were then recorded. Next, the beam scanner was moved along the beam optical axis, through the sharp focus position, until beam radii $W_{2 x}$ and $W_{2 y}$ of values equal to the previously measured $W_{1 x}$ and $W_{1 y}$ values were located. The position $z_{2}$ of these beam radii measurements was then recorded*. The sharp focus position $z_{0}$ was then determined from these measurements by taking the average of the two position measurements $z_{1}$ and $z_{2}$ with respect to the lens. Finally, the beam scanner was positioned at the waist location $z_{o}$ and the beam minimum waists $W_{o x}$ and $W_{o y}$ were measured.

It is from these measurements that the focused laser's Gaussian beam parameters were determined. It can be shown that the real-beam divergence angle $\Theta$ is given by [13]

$$
\Theta=\sqrt{\frac{W_{1}^{2}-W_{o}^{2}}{\left(z_{1}-z_{o}\right)^{2}}} .
$$

Using Eq. (2.11), the Rayleigh ranges for the real and embedded Gaussian beams were determined. The calculated beam parameters for the focused beam were then used to backwards propagate the beam through the waist creating lens and determine the Gaussian parameters for an apparent beam. Equations (2.5), (2.6) and (2.1) allowed the location $S$ and radius $w_{o}^{\prime}$ of the apparent beam to be determined (see Fig. 3.7). The

* It should be noted that, for semiconductor diode lasers, the position $z_{2}$ can be different for the beam in the two orthogonal planes if the beam is not correctly collimated since the beam will be diverging in one plane while it is converging in the other. The position $z_{2}$ can also vary for highly astigmatic sources such as gain guided laser diodes ${ }^{[20]}$. 


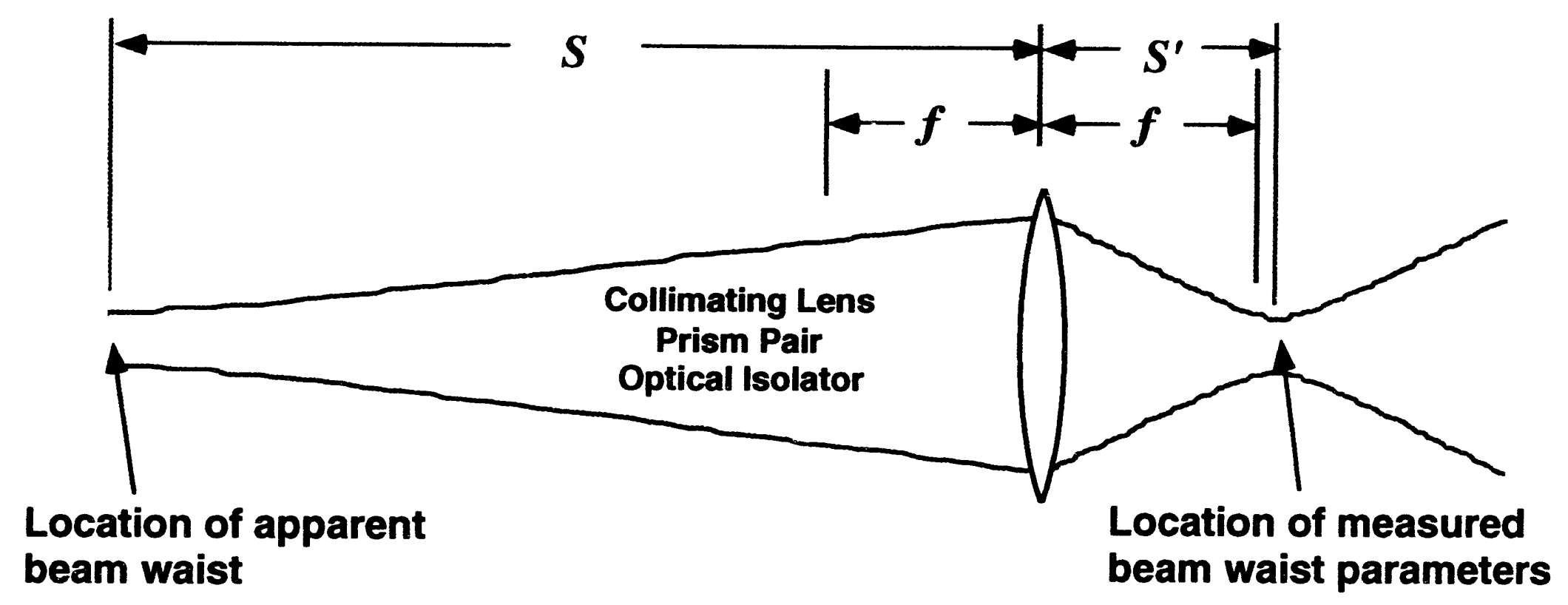

Figure 3.7 Geometry used to determine the apparent beam waist from the diode laser. Using this geometry, the previous optical elements can be ignored as if the beam were originating from a position at $S$. 
location of the waist is given with respect to the lens position which was carefully marked for later reference. It is the location and waist of this apparent beam which were used to develop the mode-matching relationship between the laser beam and the cavity. This apparent beam concept allows the previous optical elements (i.e. the collimating lens, the prism pair and the optical isolator) to be ignored when developing the mode-matching conditions.

\subsection{Fabry-Perot Cavity Characterization}

Two confocal cavity configurations were used in these experiments: an off-axis geometry (see Fig 3.8 a) and a normally incident geometry (see Fig. 3.8 b). Initially, the off-axis geometry was used to develop a fundamental understanding of the confocal cavity and to qualitatively investigate the effects of resonant optical feedback on the laser diode. The off-axis confocal cavity allows the diode laser to be geometrically isolated from nonresonant feedback from the cavity while allowing the resonant feedback to get back to the diode laser. After these preliminary experiments were completed, then the cavity was adjusted to be a normally incident geometry which was optically isolated from the laser source. This geometry was used to measure the characteristics of the cavity, develop a scheme for frequency-locking the laser to the cavity and to perform the pumped-cavity SHG experiments. For both of these geometries, detectors were positioned to measure the transmission and reflectance characteristics of the cavity.

For the normally incident cavity experiments (see Fig 3.9), the first cavity used was a 5 $\mathrm{cm}$ confocal cavity with mirror reflectivities of $R_{1}=95.4 \%$ and $R_{2}=99.94 \%$. These values were obtained experimentally. From the measurements of the collimated laser beam parameters, it was determined that a $150 \mathrm{~cm}$ focal length lens would work well for modematching to this cavity. The first experiment was performed to determine if indeed this 
(a)
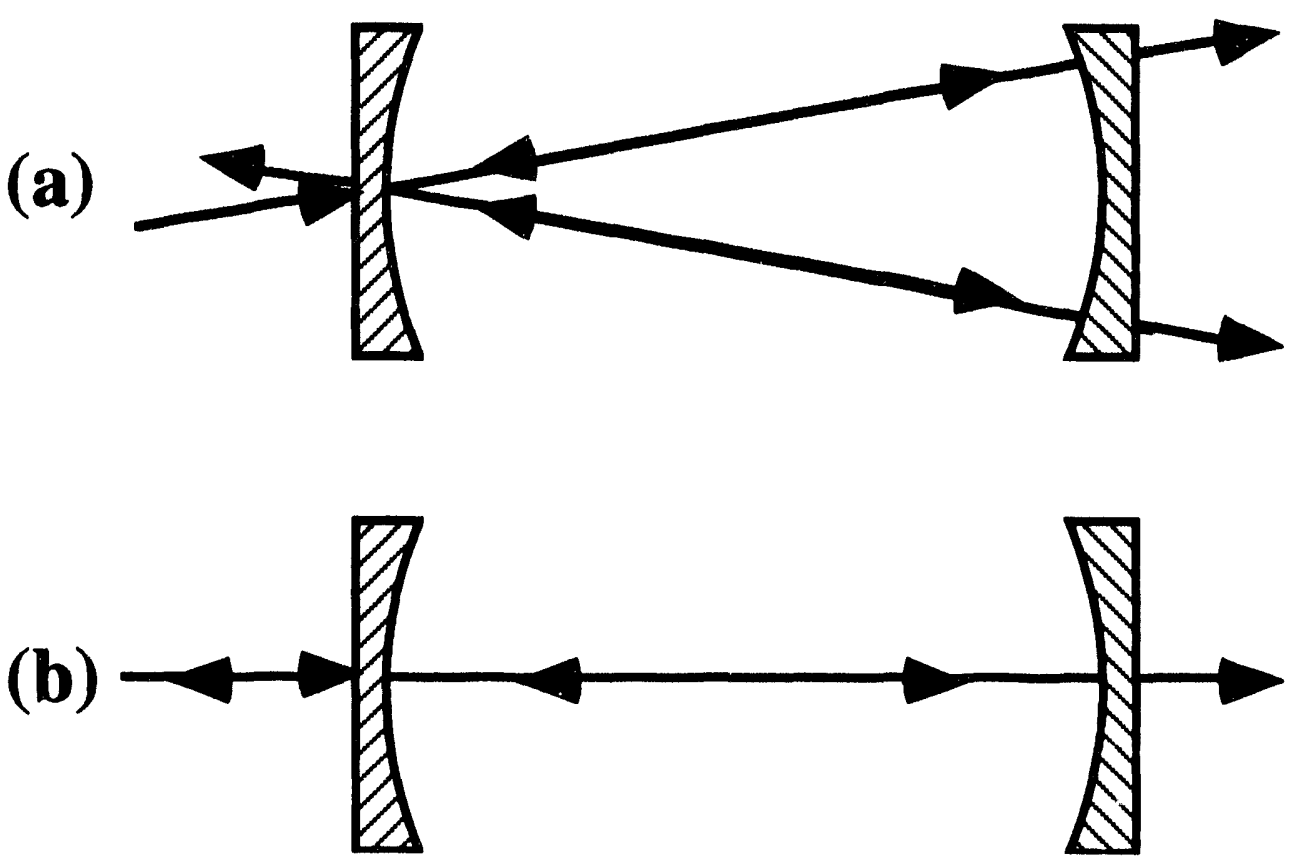

Figure 3.8 Two geometries for the confocal cavities used in the experiments leading up to and including the pumped-cavity second harmonic generation, (a) offaxis confocal cavity, (b) normal incidence confocal cavity.

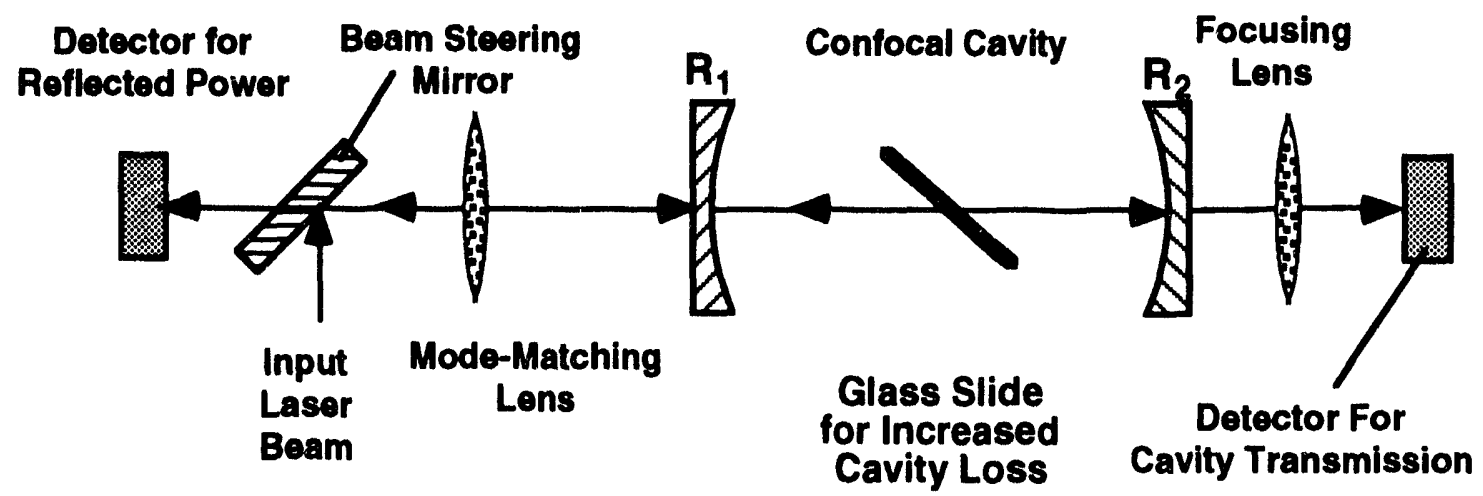

Figure 3.9 Configuration used in experiments to develop a fundamental understanding of confocal cavities. 
calculation was correct and good mode-matching was achieved. This experiment was performed with the cavity empty and a piezo-electric transducer was used to vary the cavity length periodically in a linear fashion. The transmission characteristics of the cavity were measured by focusing the light transmitted through the cavity onto a photodetector. The detector signal showing the cavity transmission characteristics was then captured and stored using a digital oscilloscope.

Next, the effect of impedance matching the cavity was analyzed. Since the selection of mirrors available was limited, to study this effect when the loss within the $5 \mathrm{~cm}$ confocal cavity was varied. Again the cavity length was modulated and the resulting reflection and transmission characteristics from the cavity were recorded. Two sets of measurements were made: the first set was made with the empty cavity while the second set was made with additional losses introduced into the cavity. The loss was introduced into the cavity by insertion of a thin glass slide into the cavity (see Fig 3.9). Since the slide was thin, only minor adjustments in mirror alignment were required to correct for any beam offsets caused by the slide. These measured characteristics were then used to determine the cavity losses and to compare these results to those predicted from the models ${ }^{[7]}$.

\section{$3.4 \quad$ Frequency-Locking}

Next, a system was established which would allow frequency-locking of the laser to the cavity length (see Fig. 3.10). It was assumed that the laser wavelength was the most rapidly varying parameter within the experimental configuration and thus was chosen as the parameter to control to obtain stable frequency-locking. An $11 \mathrm{kHz}$ sinusoidal modulation signal was applied to the laser current to dither the laser wavelength around a

center wavelength $\lambda_{o}$ by an amount $\pm \Delta \lambda$ which was small with respect to $\lambda_{o}$. The cavity was adjusted to be near the maximum resonance condition and, as expected, the 


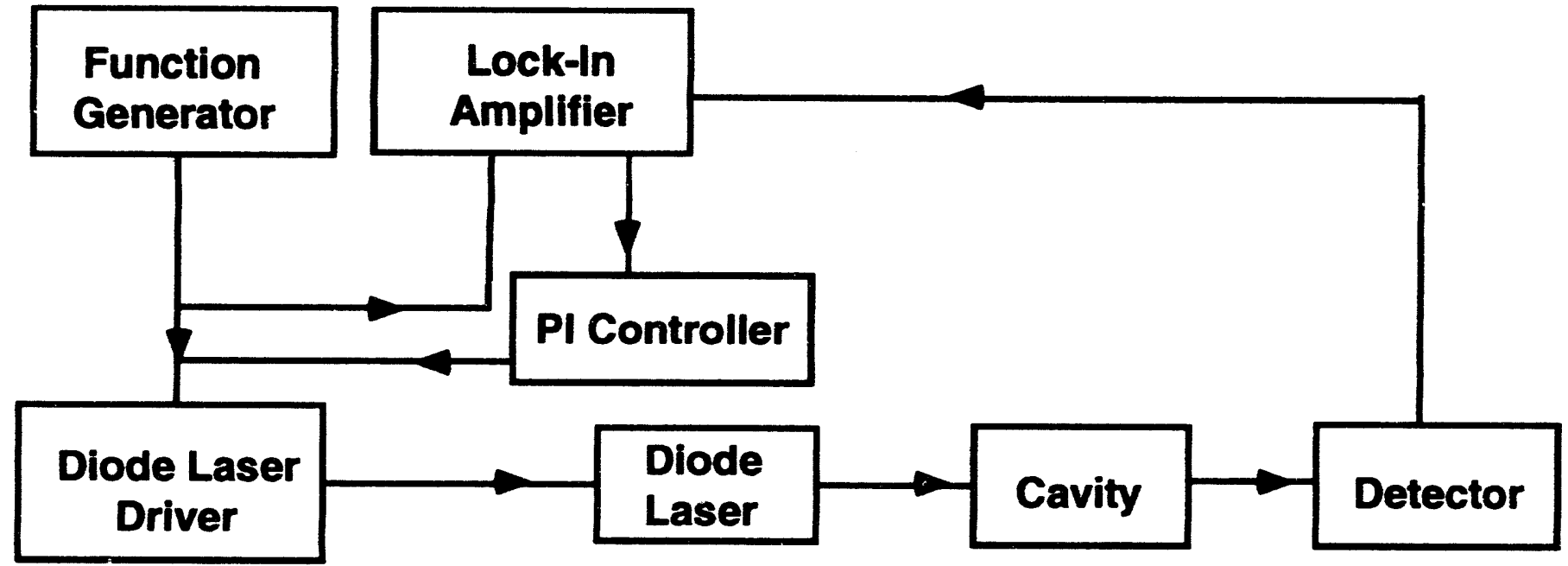

Figure 3.10 Block diagram of scheme used to "frequency-lock" the diode laser wavelength to the cavity length. 
transmitted signal obtained at the detector sinusoidally varies at the modulation frequency. The peak-to-peak amplitude of the detected signal decreases as the laser wavelength approaches the resonant wavelength of the cavity. The detector signal was then input to a lock-in amplifier, as was the modulation signal which was used to provide a reference signal for the lock-in amplifier. The lock-in amplifier then provides, as an output signal a dc voltage that is proportional to the peak-to-peak amplitude of the detected ac signal and possesses a sign which is related to the phase difference, $\Delta \phi$, between the detected signal and the reference signal. For this scheme, $\Delta \phi$ has two possible phase conditions:, $\Delta \phi=0$ and $\Delta \phi=\pi$. For these conditions, the sign of the output signal was positive for $\Delta \phi=0$ and negative for $\Delta \phi=\pi$. The output signal from the lock-in amplifier was the error signal which was used to lock the laser wavelength to the cavity length. This error signal was input to a proportional plus integral (PI) controller which allowed the gain and high frequency response of the error signal to be varied. The error signal from the PI controller was then input to the diode laser power supply to vary the laser current, and thus the laser wavelength, to maintain the center laser wavelength $\lambda_{o}$ at the maximum resonance position of the cavity.

To implement the feedback control scheme mentioned, it was first necessary to build the PI controller. This controller allowed both the dc gain and the bandwidth of the output to be varied in increments of 3.3 times the previous uncontolled value. Shorting type switches were used for both of these adjustments so that the proportional and integral values could be adjusted during operation without losing the cavity lock. The PI controller also had a built in offset adjustment which was used to zero the error signal when the modulation on the detector was small or the cavity transmission was negligible. This offset adjustment could also be used to lock the laser to positions on either side of resonance. 
The parameters required to obtain stable feedback control to frequency-lock the laser to the cavity were determined experimentally. Initially, the $\mathrm{dc}$ gain and the bandwidth of the PI controller were adjusted to their smallest values. Next, with the error signal from the lock-in amplifier input to the PI controller, the cavity length was adjusted to be approximately on resonance. This adjustment was performed by manually varying the voltage on the piezoelectric transducer which subsequently provided gross adjustment of the cavity length. After the cavity was brought to be near resonance, then the dc gain of the PI controller was increased while the detected signal, due to the cavity transmission, was observed on an oscilloscope. The dc gain was adjusted in steps until the signal observed on the oscilloscope became unstable and began to oscillate. At that point, the dc gain was reduced one position. Next the bandwidth of the PI controller was increased until the detected signal again became unstable. The bandwidth position was then adjusted down one position where it was again stable. These stable parameters were then used to perform frequency-locking to the cavity during these experiments.

\subsection{Single Pass Second Harmonic Generation}

Before beginning the pumped-cavity SHG experiment, a single-pass SHG experiment was performed to characterize the NLO crystal performance (see Fig. 3.11). For this experiment, the $\mathrm{NLO}$ crystal used was $\mathrm{KNbO}_{3}$ which had a $3 \times 3 \mathrm{~mm}$ square aperture and was $5 \mathrm{~mm}$ long. Phase matching in the crystal was accomplished by tuning the crystal temperature until maximum conversion was obtained. To control the crystal temperature, a small aluminum cell was built which maintained contact with the crystal on three sides. This cell was mounted to a thermo-electric cooler and a calibrated thermistor was attached to the side of the aluminum cell. To insulate the cell from air currents a plastic cover was machined to fit over the top of the cell. In order to fit into the small cavities 


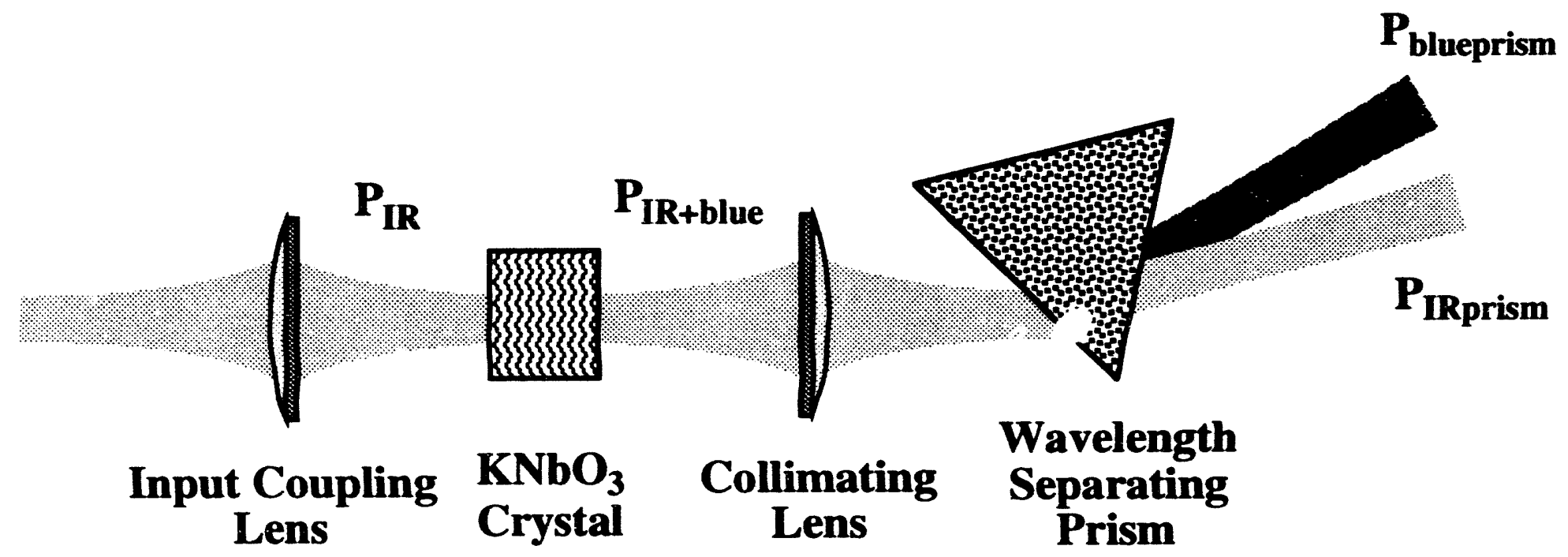

Figure 3.11 Schematic representation of apparatus used in single-pass SHG experiment. The subscripted P variables indicate the locations of the various power measurements. 
used in these experiments, the cell was made to be only slightly larger than the crystal itself. Finally, a commercially available PID temperature controller ${ }^{[25]}$ was used to stabilize the crystal temperature. The temperature controller was specified to maintain the crystal temperature to $\pm 0.01^{\circ} \mathrm{C}$. Since the $\mathrm{KNbO}_{3}$ crystal allows noncritical phase matching, i.e. along the crystal axis, walk-off of the SH beam was not a problem. A photograph of the crystal holder is shown in Figure 3.12.

The beam was focused to produce waists of 25 and $22 \mu \mathrm{m}$ in two orthogonal planes which, due to astigmatism, were located at positions $1.5 \mathrm{~mm}$ apart. The average waist due to these two orthogonal waists was used in calculations and had a Rayleigh range of $4.54 \mathrm{~mm}$ which was approximately equal to the crystal length. The power was measured using a Newport model 815 power meter which was calibrated to account for the difference in detector responsivity at the fundamental and SHG wavelengths. A prism was used to separate the fundamental beam from the SH beam. To determine the optical losses to the beam after the NLO crystal, the total beam power was measured in several locations. To account for these losses, two assumptions were made: (1) the power of the SH light was negligible with respect to the fundamental wavelength and (2) the losses at the SH wavelength are approximately the same as those for the fundamental wavelength. Since the SH power was small with respect to the fundamental power and the optics were made of fused silica and uncoated and hence insensitive to wavelengths over the $0.4-0.9$ $\mu \mathrm{m}$ range, these assumptions were valid. From these measurements, a calibration factor was determined which allowed the measured SHG power to be scaled to account for the optical losses to the beam. Substitution of these measured values into Eq. (2.29) allowed the nonlinear optical coefficient of the $\mathrm{KNbO}_{3}$ crystal to be determined experimentally. 


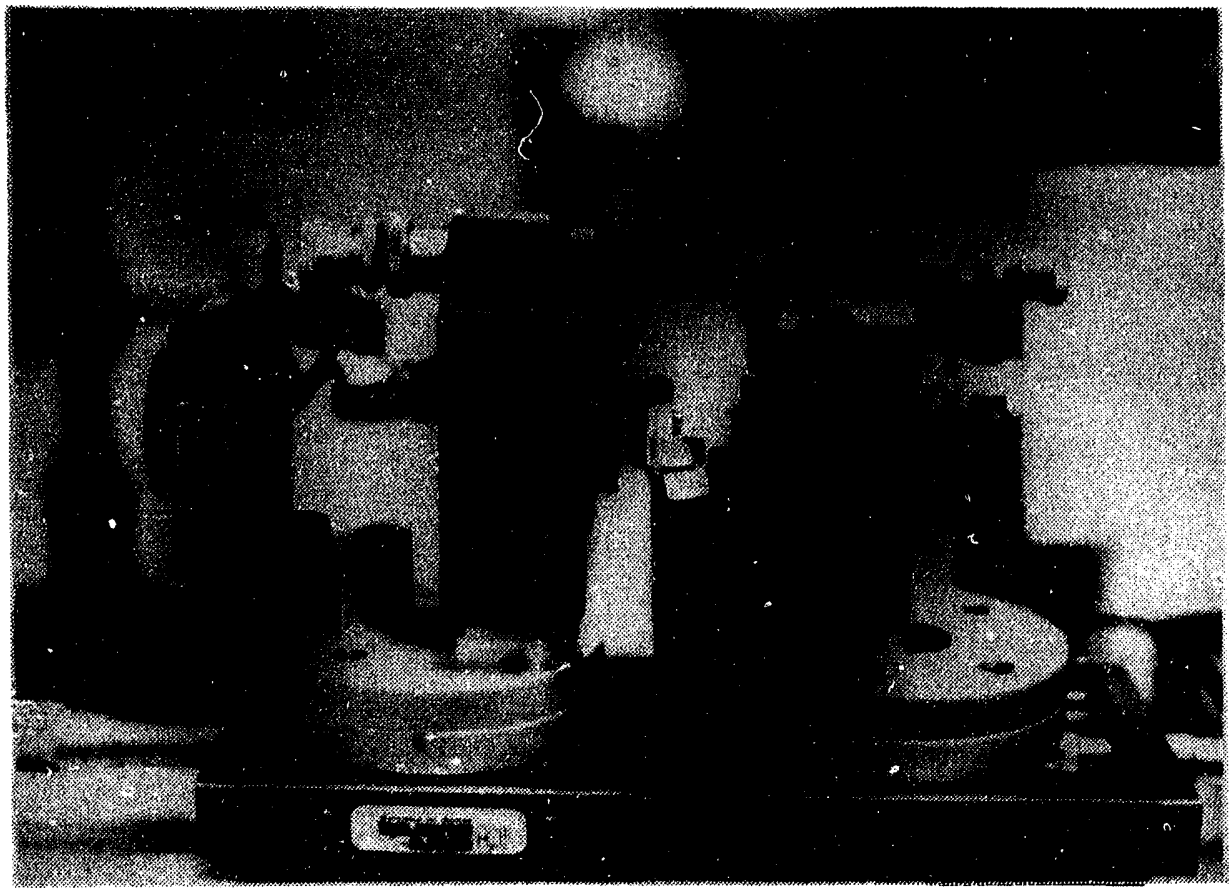

Figure 3.12 Photograph of cell built to house $\mathrm{KNbO}_{3} \mathrm{NLO}$ crystal for temperature controlled phase-matching. 


\subsection{Pumped-cavity Second Harmonic Generation}

For this portion of the experiment, all of the details learned about Gaussian beam parameters, confocal cavities, frequency-locking, etc. were combined. Results from preliminary experimentation indicated that the $5 \mathrm{~cm}$ cavity would not be optimum but that a $2.5 \mathrm{~cm}$ confocal cavity would improve the performance of the pumped-cavity SHG. Although some preliminary results were obtained using the previously described 5 $\mathrm{cm}$ confocal cavity, most of the reported results were obtained using the $2.5 \mathrm{~cm}$ cavity .

The cavity configuration for the pumped-cavity SHG is shown in Figure 3.13. The input mirror $\mathbf{R}_{1}$ was anti-reflection coated on the plane input surface and had a reflectivity of $97.8 \%[26]$ on the spherical side of the mirror for the fundamental laser wavelength. The output mirror $R_{2}$ had a reflectivity of $R_{2}=99.85 \%[26]$ on the spherical mirror surface at the fundamental laser wavelength and a reflectivity of $14 \%$ for the SH light. The plane output side of the output mirror was uncoated. The input mirror reflectivity was chosen to match the expected losses in the cavity due absorption, scattering, SHG, etc. and was obtained by solving Eq. (2.35). For this calculation, it was assumed that all of the laser power is coupled into the pumped-cavity when the input mirror reflectivity $R_{I}$ exactly matches the cavity reflectance parameter $R_{m}{ }^{*}$.

Detectors were positioned to measure the reflected and transmitted power from the cavity for process characterization. The signal due to the cavity transmission was also used for feedback control. On the output side of the cavity, a dichroic beam splitter was used to separate the fundamental and SH beams. A calibrated Newport model 815 power meter was positioned to measure the SH light output power. On the input side of the cavity, a long pass filter with a $500 \mathrm{~nm}$ cut-off wavelength was utilized to eliminate the SH light incident onto the detector used measure the characteristics of the reflected beam

\footnotetext{
* For the numerical solution and a detailed description of these calculations, refer to Appendix A.
} 
Power Meter for SH Light

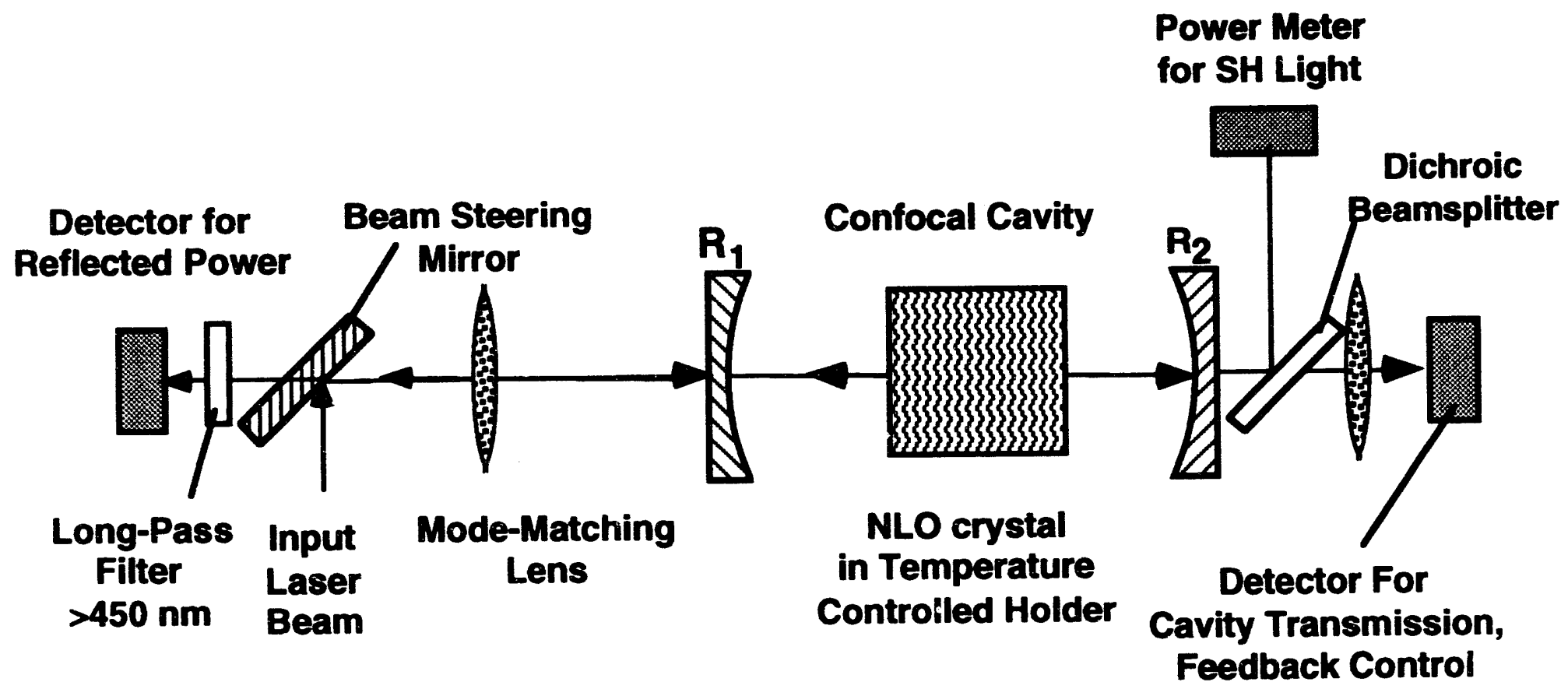

Figure 3.13 Pumped-cavity second harmonic experiment configuration. 
from the cavity. The NLO crystal used in these experiments was a $7.5 \mathrm{~mm}$ long $\mathrm{KNbO}_{3}$ crystal. This crystal was obtained after the preliminary results obtained using the $5 \mathrm{~mm}$ long crystal indicated that the longer crystal would provide a higher nonlinear loss to the second harmonic and thus better impedance match to the available mirrors. This crystal was anti-reflection coated for both the fundamental and $\mathrm{SH}$ wavelength with reflectivities of 0.015 and $0.02 \%$ at the fundamental wavelength and 0.2 and $0.4 \%$ at the SH wavelength for the two crystal surfaces[27].

To insure that the power measured at the SH power meter was due to the SH light and not to fundamental light reflected from the dichroic beamsplitter, the power reflection coefficient for the dichroic beamsplitter was measured experimentally. For this measurement, the NLO crystal temperature was increased to a position where no significant SHG occurred. The fundamental power transmitted through the cavity was measured just outside the output mirror of the cavity while the cavity was resonant. Then the power meter was repositioned to measure the fundamental light reflected from the dichroic beamsplitter. This measurement was taken in the location where the power meter would be located to measure the SH light reflected from the dichroic beam splitter. The ratio of these two measurements allowed the power reflection coefficient of the beamsplitter at the fundamental wavelength to be determined.

To perform the pumped-cavity SHG experiment, the cavity was aligned to obtain maximum mode-matching which was determined by scanning the cavity length. After the cavity was aligned, then the crystal, which was mounted on a 5 axis ( 3 translation, 2 tilt axes) positioner, was brought into the beam path. The crystal was then aligned to maximize the finesse of the cavity and eliminate any beam walk-off due to crystal misalignment. For this procedure, the NLO crystal temperature was elevated to avoid producing SHG. After the crystal was aligned, then the position of the front mirror, 
which was mounted on a linear positioning stage, was adjusted to increase the cavity length until the confocal condition was obtained. At this point the cavity mirrors were again adjusted to optimize mode-matching. Finally, the crystal temperature was adjusted to tune the crystal to optimize SHG output power.

Several experiments were performed to characterize the pumped-cavity SHG process. After separation of the fundamental and SHG beam, measurements were taken to determine the beam quality of the blue light output from the cavity. For these measurements, the separated SH light was passed through a lens to create a waist and the intensity distribution of the focused beam was measured at several locations along the beam optical axis. The intensity distribution was measured in two orthogonal planes which corresponded to the earlier mentioned planes parallel and orthogonal to the diode junction plane. The beam radii measurements and their position along the optical axis with respect to a reference plane were recorded. These measurements were made at 1 $\mathrm{mm}$ increments over a distance of $14 \mathrm{~mm}$. The measured values for the beam radii were then used to determine the beam quality of the SH laser light in the two orthogonal planes. These measurements were made for unidirectional SH output powers from the cavity of 2.5 to $6.5 \mathrm{~mW}$.

Also of particular interest was the determination of the temperature tuning bandwidth for optimum phase matching in the $\mathrm{KNbO}_{3} \mathrm{NLO}$ crystal. These measurements were made by keeping the fundamental laser power constant while varying the NLO crystal temperature over a relatively large (with respect to SHG output power variations; temperature range. The $\mathrm{SH}$ output power from the cavity was measured as the temperature of the NLO crystal was varied. These measurements were made at fundamental input powers from 35 to $60 \mathrm{~mW}$. During this portion of the experiment, the actual resistivity of the control thermistor was monitored and a calibration curve supplied by the manufacturer ${ }^{[28]}$ was used to determine the actual crystal temperature. 


\subsection{Summary}

This chapter covered the work and various experiments performed which led to the pumped-cavity SHG experiment. The pumped-cavity SHG experiment was broken down into key areas which were essential to the experiment. These areas included laser beam modification and characterization, Fabry-Perot cavity characterization, the frequencylocking system and single-pass SHG. To achieve optimum results from the pumpedcavity SHG experiment, each of these areas was investigated independently and a sufficient amount of time was spent with each area until it was well understood.

The laser beam cross-sectional distribution was modified to be compatible with the circular symmetry of the cavity and the diode laser was optically isolated to eliminate undesirable effects due to optical feedback to the diode. Next, measurement techniques for determining the laser's Gaussian beam parameters and linewidth were discussed and the methods used in characterization of the empty confocal cavity were presented.

A schematic representation of the frequency-locking system developed to lock the laser frequency to the cavity frequency was shown. The scheme used to generate the feedback control error signal was discussed in detail as was the implementation of the feedback control.

The experimental configuration and implementation for the single-pass second harmonic generation experiment was discussed. The primary focus of this experiment was to provide a comparison between the single-pass conversion efficiency and the pumpedcavity conversion efficiency. 
Finally, the experimental configuration for pumped-cavity SHG was presented. This section discussed the cavity configuration and the various methods used to evaluate performance. The parameters investigated in the characterization experiments include SHG conversion efficiency measurements, phase-matching temperature bandwidth determination and SH beam quality measurements. 


\section{CHAPTER 4}

\section{Experimental Results and Discussion}

\subsection{I aser Beam Parameters}

After the output beam from the diode laser was optically manipulated to be approximately circular, then the beam parameters associated with the SDL-5422-H1 diode laser were measured. These measurements included determination of the laser linewidth and its Gaussian beam parameters.

In order to match the diode laser to the cavities used in these experiments it was necessary to determine the laser's bandwidth characteristics. To make these measurements, a $25 \mathrm{~cm}$ confocal cavity was used and mode-matching was not optimized. It was only necessary to mode-match approximately to the cavity so that the transmission peaks corresponding to the even symmetry transverse modes could be differentiated from the odd symmetry transverse modes. The transmission characteristics from the cavity obtained for the approximate mode-matched condition are shown in Figure 4.1.

When optical feedback was applied to the laser, a finesse of 107 was measured. The theoretical value for the cavity free spectral range was calculated to be

$$
\Delta v=6 \times 10^{8} \mathrm{~Hz}
$$

and the time corresponding to the measured cavity free spectral range was

$$
\Delta t=2.489 \mathrm{~ms} .
$$




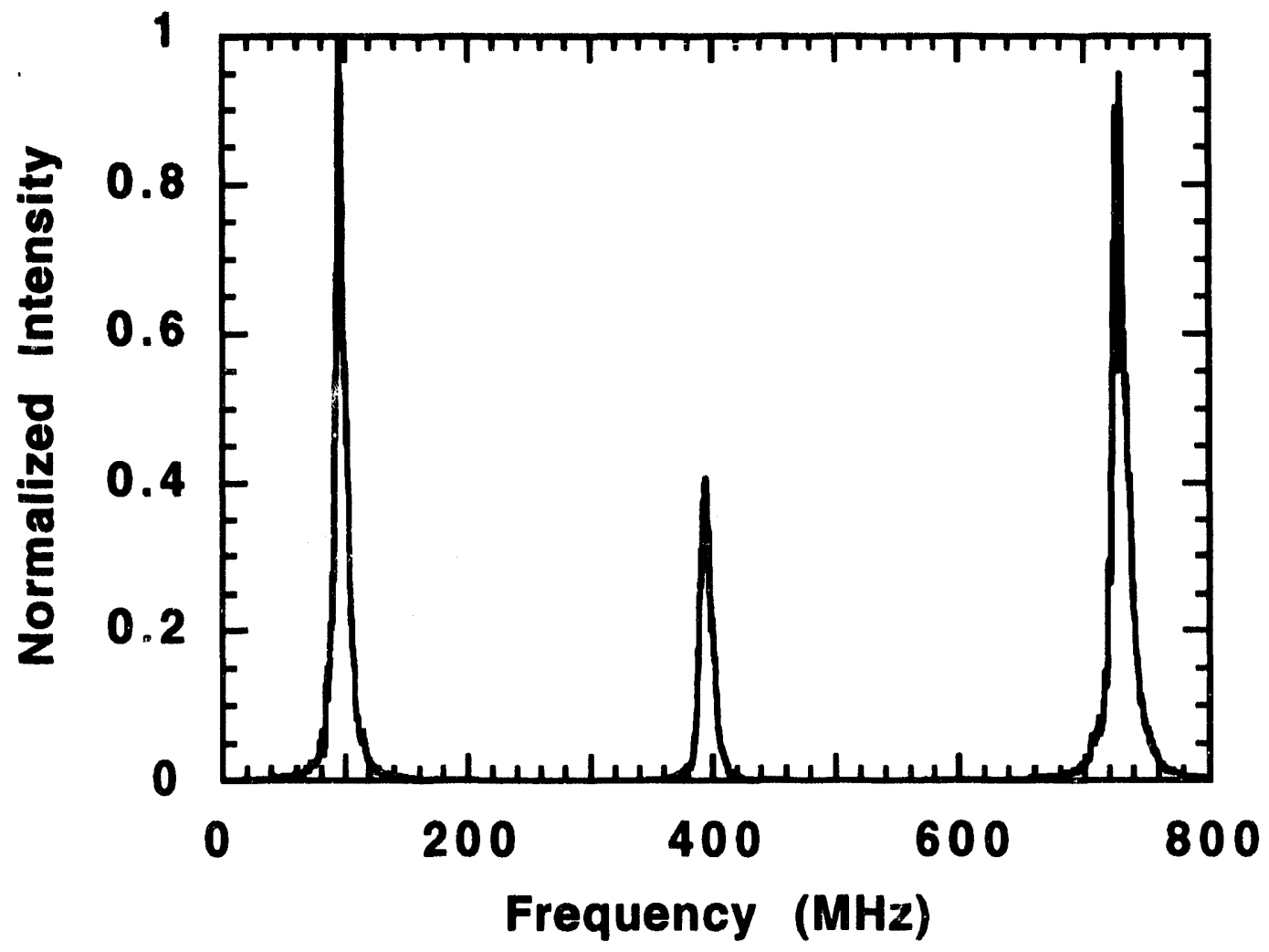

Figure 4.1 Measured transmission characteristics obtained from scanning $25 \mathrm{~cm}$ confocal resonator showing poor mode-matching condition. Note that the larger peaks correspond to excitation of the even-symmetry cavity transverse modes while the shorter peaks correspond to excitation of the odd-symmetry cavity transverse modes. 
The ratio of Eqs. (4.1) and (4.2) was calculated to determine a calibration factor $a$ relating the measured values in time to values in frequency to be

$$
a=\left(\frac{\Delta v}{\Delta t}\right)_{\text {caviry }}=2.411 \times 10^{11} \frac{\mathrm{Hz}}{\mathrm{s}} .
$$

For this particular cavity, the minimum cavity resolution was determined to be $5.79 \mathrm{MHz}$. Thus, if the laser linewidth became smaller than $5.79 \mathrm{MHz}$, it could not be measured accurately with this particular cavity.

Finally, with the cavity aligned and calibrated, the linewidth of the SDL-5422-H1 diode laser was measured. The measured laser linewidth, plotted as a function of laser output power, is shown in Figure 4.2. From this graph, it can be seen that the laser linewidth is monotonically decreasing with increased power as expected $[3,20]$. There were some limitations encountered with these measurements. Although the diode laser used has a maximum rated output of $150 \mathrm{~mW}$, our measurements were limited to powers below 100 $\mathrm{mW}$ by the minimum resolution of the cavity. As the power was increased beyond 85 $\mathrm{mW}$, all linewidth measurements obtained were approximately equal to the cavity minimum resolution and thus were not shown in Figure 4.2.

As the cavity minimum resolution becomes much less than the linewidth of the laser, then the cavity transmission peak acts as an impulse function with respect to the laser spectral distribution. When this condition is satisfied, then the true structure of the laser spectral distribution can be obtained. When our laser was operating at $14 \mathrm{~mW}$ output power, the laser linewidth was approximately three times the minimum resolution of the cavity. With this relatively small difference between the cavity resolution and the laser linewidth the uneven structure of the spectral distribution is evident (see Figure 4.3). 


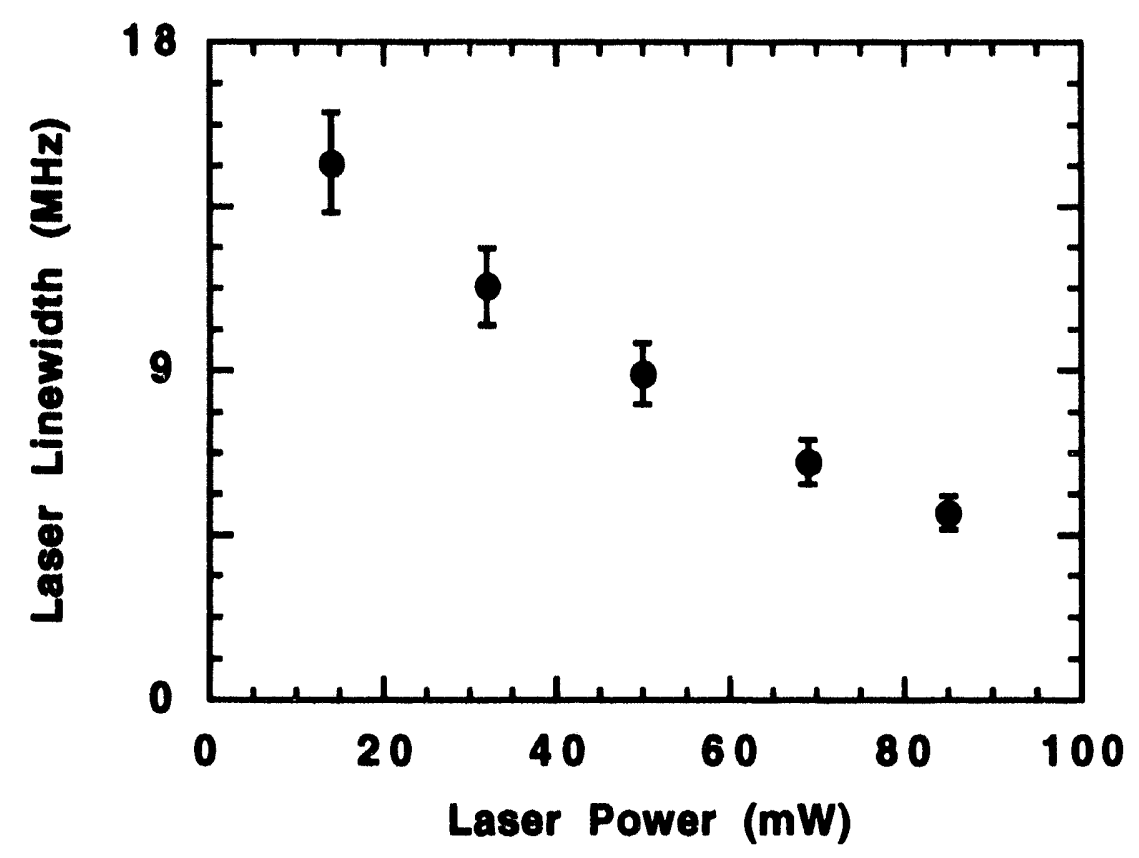

Figure 4.2 Measured linewidth for Spectra Diode Labs model SDL-5422 laser diode as a function of laser output power.

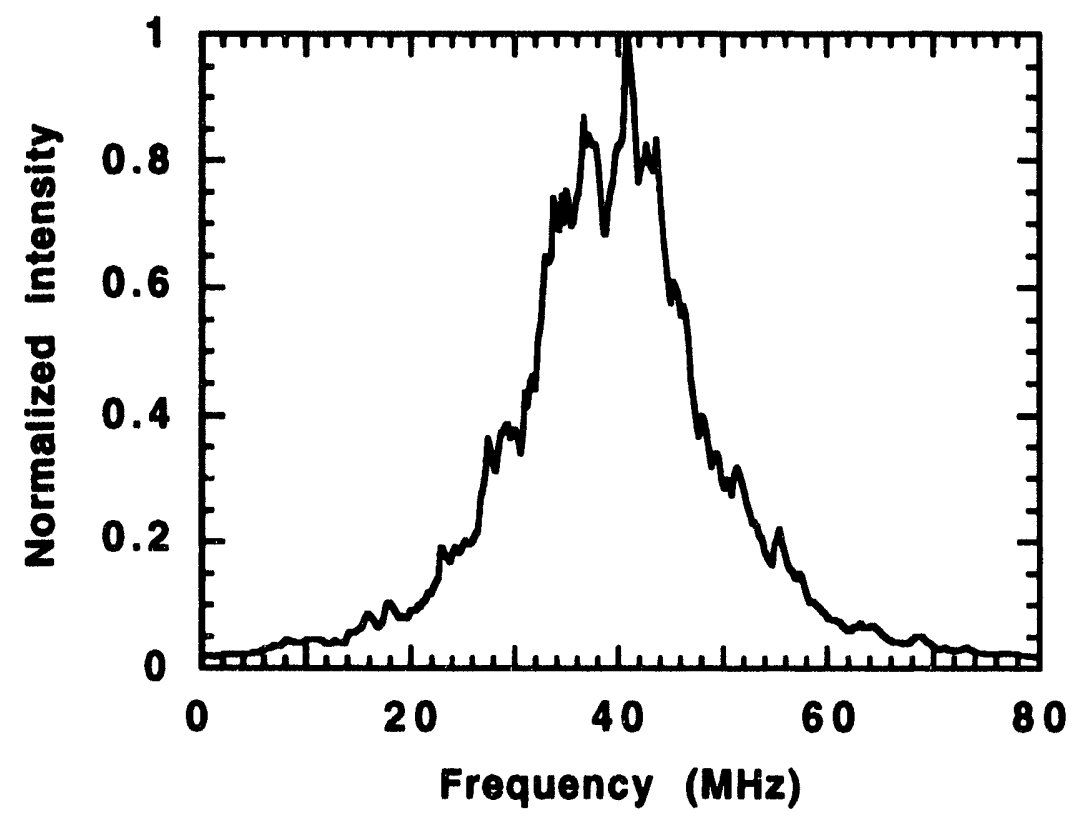

Figure 4.3 Measured structure of the laser spectral distribution at $14 \mathrm{~mW}$ laser output power. 
The next step was to measure the laser's Gaussian beam parameters. The beam was passed through a lens with a focal length of $250 \mathrm{~mm}$ to produce a waist. Then the beam radius was measured at two positions $z_{1}$ and $z_{2}$ (see Fig. 3.5) on opposite sides of the sharp focus position, to find the location $z_{0}$ of the beam minimum waist. After the beam minimum waist was located, its radius was then measured. As mentioned previously, these parameters were measured in the directions parallel and orthogonal to the diode junction. These measured beam radii for the real beam are given in Table 4.1.

From these measured values, the Gaussian beam parameters for the real-laser beam were determined using the formalism developed in the literature $[12,13]^{*}$. These parameters included determination of the Rayleigh range, the beam divergence angle, the beam quality parameter and the embedded Gaussian beam waist contained within the real beam. These calculated values are given in Table 4.2. Using these calculated values, the beam was then backwards propagated through the waist-creating lens to determine an apparent beam waist, Rayleigh range and the location of the apparent beam waist with respect to the waist producing lens. These values, also given in Table 4.2, were then used to match the real beam to the various cavities used during these experiments. It should be noted that in determining the value corresponding to the embedded Gaussian beam waist, the average value of the beam waist in the $x$ - and $y$-directions was used. For this laser, the beam quality parameter $\mathrm{M}^{2}$ was determined to be 1.37 and 1.51 along the $\mathrm{x}$ - and $\mathrm{y}$ axes respectively.

\subsection{Fabry-Perot Cavity Parameters}

Using the values obtained for the apparent beam Gaussian parameters, relationships for mode-matching the embedded Gaussian beam to the various cavities were developed.

* For a complete description of the procedure used in these calculations, refer to Appendix B. 


\begin{tabular}{|c|c|c|}
\hline Distance from Lens $(\mathrm{mm})$ & $W_{x}(\mu \mathrm{m})$ & $W_{y}(\mu \mathrm{m})$ \\
\hline 242 & 91.35 & 100 \\
\hline 263.8 & & 100 \\
\hline 263.9 & 91.35 & \\
\hline 252.9 & 58 & 53 \\
\hline
\end{tabular}

Table 4.1 Measured values for real beam radii from the diode laser after optical manipulation to circularize the beam (Note that the distance measurements have an uncertainty of $\pm 2 \mathrm{~mm}$ and the waist radii have an uncertainty of \pm $1.3 \mu \mathrm{m})$.

\begin{tabular}{|c|c|c|}
\hline Laser Parameter & \multicolumn{2}{|c|}{ Calculated Values } \\
\hline $\begin{array}{c}\text { Real-Beam Divergence } \\
\text { (mrad) }\end{array}$ & Focused Beam & Apparent Beam \\
\hline $\begin{array}{c}\text { Times-Diffraction-Limited } \\
\text { Rayleigh Range (mm) }\end{array}$ & $1.4 \pm \pm 0.33$ & $0.473 \pm 0.033$ \\
\hline $\begin{array}{c}\text { Waist Location from } \\
\text { Focusing Lens (mm) }\end{array}$ & $7.8 \pm 0.5$ & $1.44 \pm 0.04$ \\
\hline $\begin{array}{c}\text { Embedded Gaussian Beam } \\
\text { Waist }(\mu \mathrm{m})\end{array}$ & $252.9 \pm 2$ & $7040 \pm 1801$ \\
\hline
\end{tabular}

Table 4.2 Calculated values for the focused laser beam and the apparent beam incident on the lens which produced focused beam values. 
The first cavity used was a confocal cavity with mirror radii of $5 \mathrm{~cm}$. Both mirrors had a thickness of $9.53 \mathrm{~mm}$ which, for a $5 \mathrm{~cm}$ confocal cavity, leads to a value of

$$
d_{L}=6.58 \mathrm{~cm}
$$

Substitution of this value, as well as the mirror radii of curvature and the beam radius at the mirror surface into Eq. (2.20), gave an apparent beam waist $w_{c}$ within the cavity of

$$
w_{c}=52.1 \mu m \text {. }
$$

It is this apparent beam waist which was used to determine the ideal mode-matching conditions. Using this value for the apparent beam waist within the cavity $w_{c}$ along with the value for the apparent beam waist due to the laser $w_{L}$ (see Table 4.2), the conditions for mode-matching were calculated from equations (2.21)-(2.23) and equation (2.25). The values obtained from these calculations are given in Table 4.3 and correspond to the quantities shown in Figure 4.4.

For this particular configuration, a lens with a $150 \mathrm{~mm}$ focal length was chosen since it was the smallest value for a standard lens which would allow the mode-matching conditions to be physically realized. From Table 4.1, it can be seen that the apparent beam waist position with respect to the lens position is $2.87 \mathrm{~m}$; however, in the actual experimental configuration the distance from the laser diode to the reference position is only $22 \mathrm{~cm}$. Thus, this value for a mode-matching lens did actually minimize the length of the experiment configuration.

After the ideal mode-matching conditions were calculated, these values were used in positioning of the various optical components utilized in the experiment. The cavity was aligned and optical detectors were used to measure both the reflected and transmitted optical power from the cavity. Figure 4.5 shows the measured transmission 


\begin{tabular}{|c|c|}
\hline Parameter & Value (mm) \\
\hline$f_{o}$ & 84.2 \\
\hline$f$ & 150 \\
\hline$S_{L}$ & 127 \\
\hline$d_{L}$ & 3310 \\
\hline$d_{2}$ & 154.7 \\
\hline$Z_{L}$ & 27.7 \\
\hline
\end{tabular}

Table 4.3 Calculated values for mode-matching to $5 \mathrm{~cm}$ confocal cavity. Note that the quantities that these values represent are shown in figure 4.4.

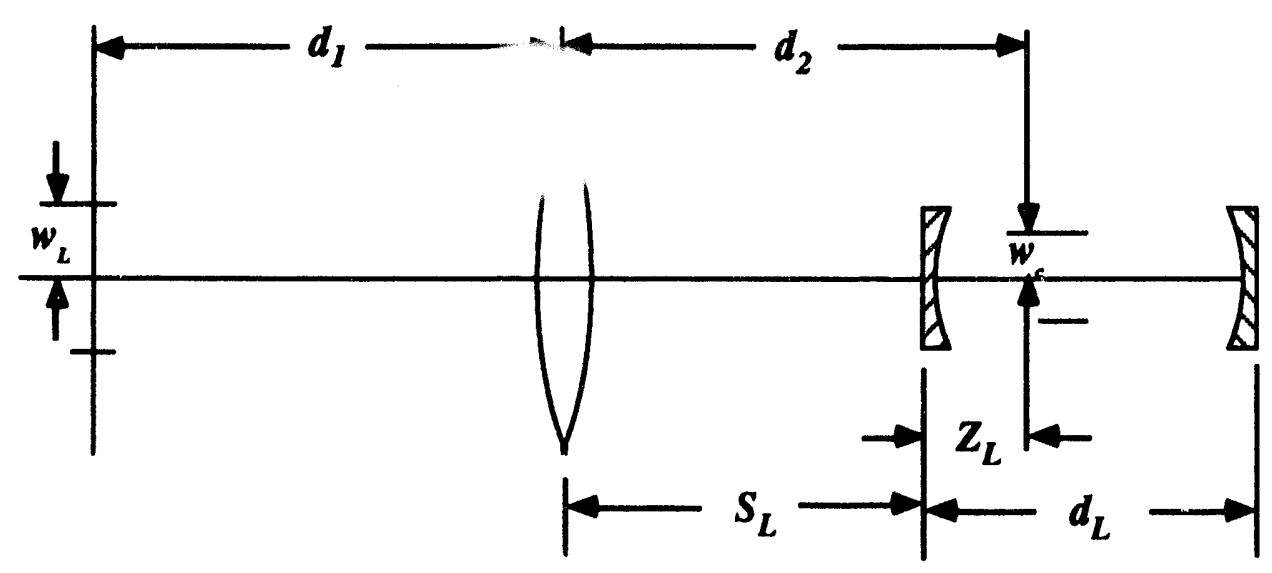

Figure 4.4 Schematic representation of geometry used to develop mode-matching conditions of a laser beam to a cavity both of which have known parameters. 


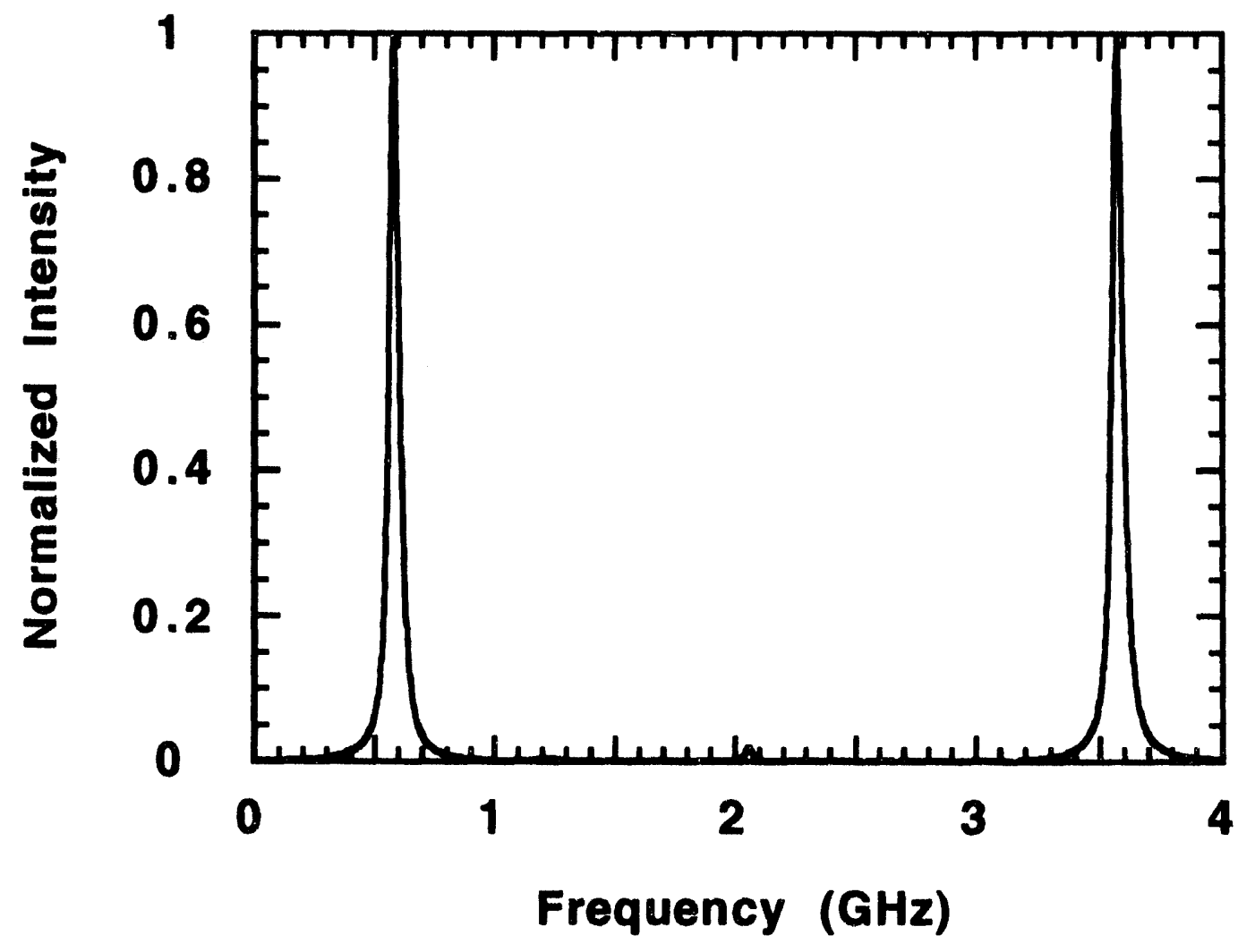

Figure 4.5 Measured transmission characteristics of $5 \mathrm{~cm}$ confocal cavity with extremely good mode-matching between incident laser beam and the cavity. 
characteristics of the scanning cavity after alignment. The two large peaks correspond to excitation of the even-symmetry transverse cavity modes and are spaced a distance identical to the cavity free spectral range. Note that the much smaller peak between two large peaks, due to excitation of the odd symmetry transverse cavity modes, is insignificant in area with respect to the larger peaks. This indicates that mode-matching between the laser beam and the cavity is extremely good.

Next, impedance-matching of the cavity was investigated by analyzing the reflections from the scanning cavity (see Fig. 4.6). For these measurements, the cavity was not scanned in a linear sense and the characteristics of modulation voltage applied to the piezo-electric transducer on the cavity were not recorded. Thus, the exact losses associated with these cavity configurations could not be calculated from measurements of the cavity finesse. However, the measured characteristics do demonstrate, in a qualitative sense, the effect of increased cavity losses on reflectance from the cavity.

The reflection characteristics from the $5 \mathrm{~cm}$ cavity with and without a piece of glass inserted into the beam path are shown in Figure $4.6 \mathrm{a}$ and $4.6 \mathrm{~b}$, respectively. As shown, when the glass is inserted into the cavity the reflection decreases. To impedance match to a cavity, i.e. have zero overall reflectance, the cavity reflectance parameter $R_{m}$ (as developed in the pumped-cavity SHG theory) must be equal to the input coupler reflectivity. Using Eq. (2.39), the normalized reflectance for this particular cavity as a function of cavity loss was calculated (see Fig. 4.7). With the cavity empty, the losses are minimized and so it is anticipated that the cavity reflectance would be relatively large due to the low reflectance of the cavity input mirror $R_{l}$. This is consistent with the results obtained for the low-loss cavity (see Fig. 4.6 a). Using the normalized reflectance value for the low-loss cavity, the empty cavity loss was graphically determined to be 09024 . Then, with the glass slide inserted into the cavity, the losses within the cavity should 


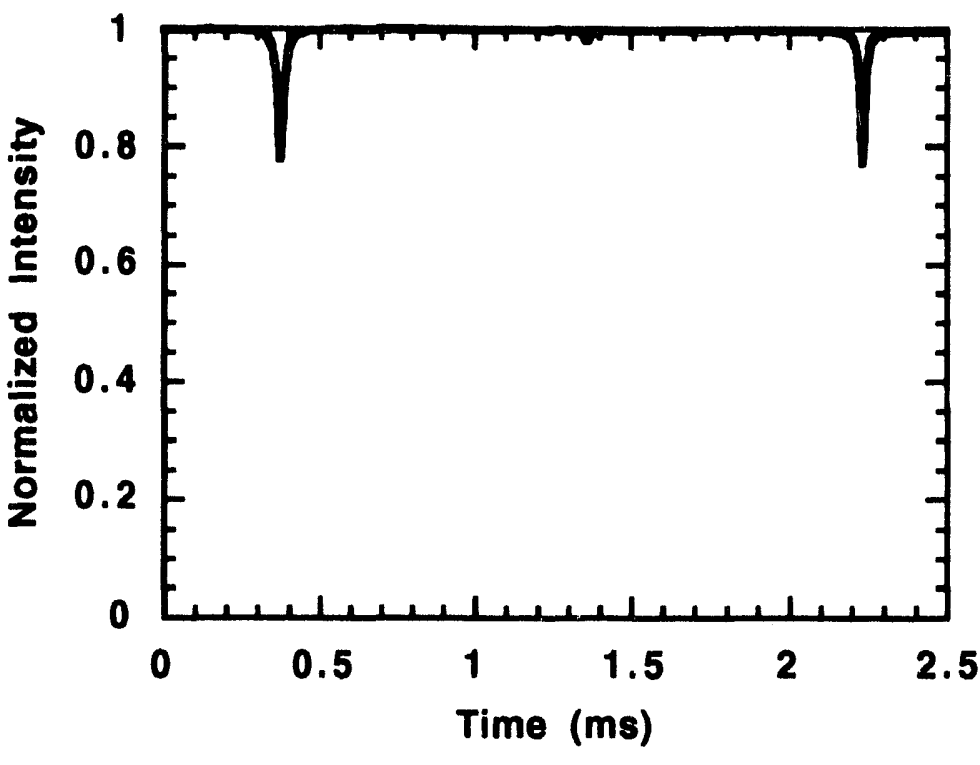

(a)

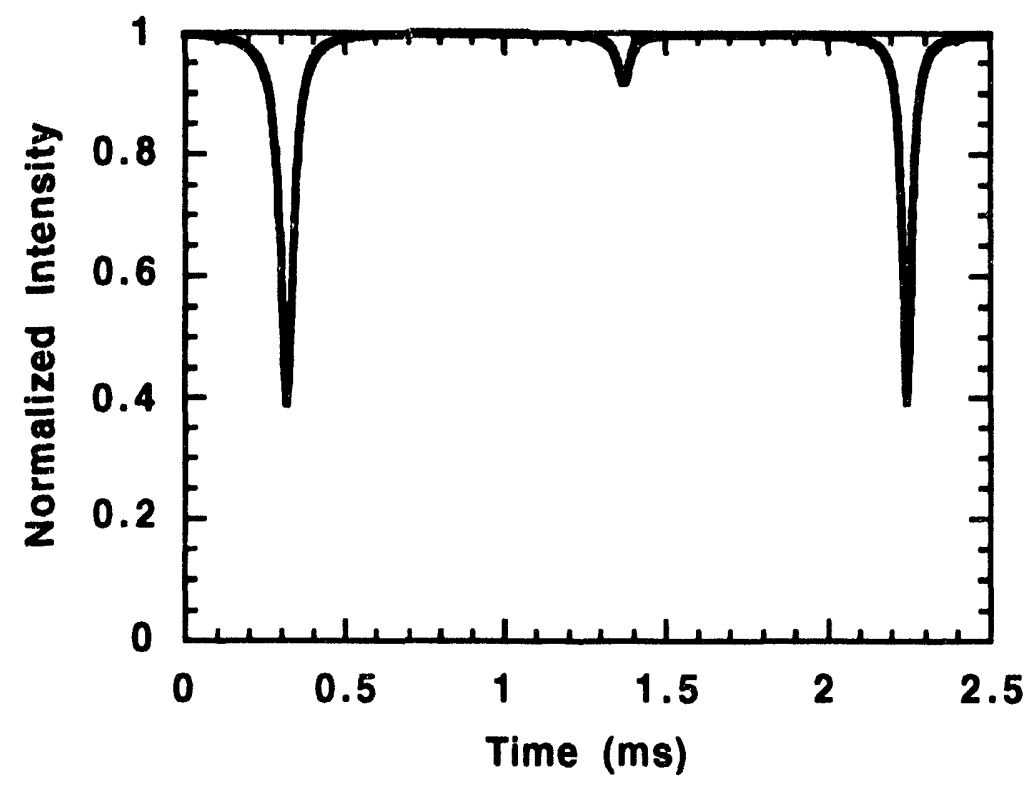

Figure 4.6 Reflection from scanning cavity with cavity losses varied: (a) empty cavity (b) glass slide inserted into cavity. 


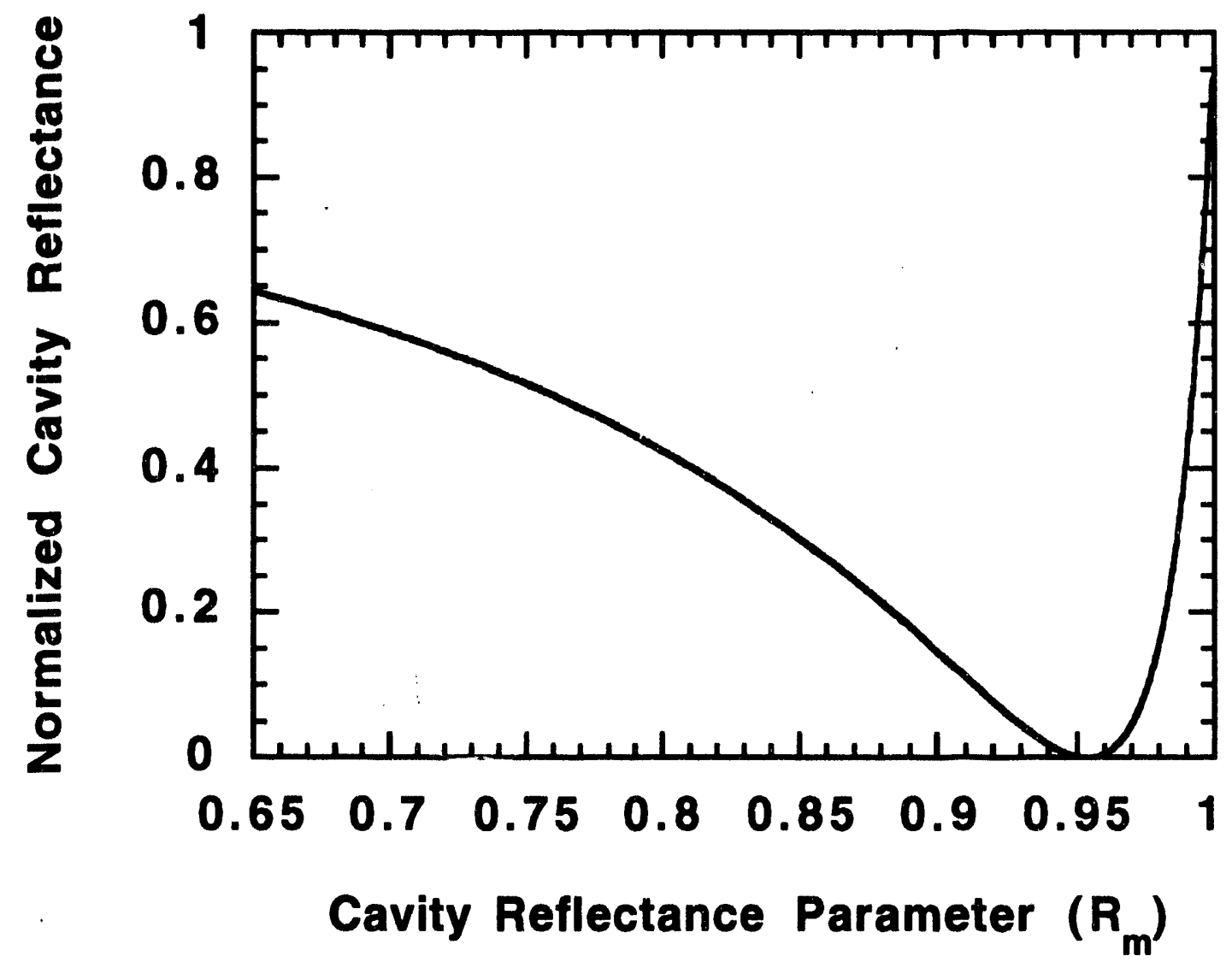

Figure 4.7 Calculated reflectance from the $5 \mathrm{~cm}$ confocal cavity as a function of the cavity reflectance parameter. 
increase significantly and thus, if these losses were not too great, the reflectance from the high-loss cavity would be expected to be less than for the low-loss cavity. This is consistent with the results obtained for the high-loss cavity (See Fig. 4.6 b). Using the normalized reflectance value for the high-loss cavity, the cavity loss was determined graphically to be either 0.0106 or 0.1854 . Of these two cavity losses, 0.1854 is the logical solution. A more accurate method for determining the cavity losses would have been to scan the cavity length linearly and measure the cavity finesse with the various cavity losses[14]. The more accuarte method was however used in determining the cavity losses for the pumped-cavity SHG experiments.

\subsection{Frequency-Locking Results}

The detector signal due to cavity transmission input to the lock-in amplifier was passed through a high pass filter with $2.63 \mathrm{kHz}$ cutoff frequency to reduce any low frequency noise associated with this signal. The full-scale sensitivity of the lock-in was adjusted to be $5 \mathrm{mV}$. The output signal from the lock-in amplifier was passed through a low pass filter with a time constant less than $1 \mathrm{~ms}$ and had a maximum amplitude of $\pm 10 \mathrm{~V}$ peakto-peak. The final values for the PI controller were a low frequency gain of 3.3 and a 3 $\mathrm{dB}$ cutoff frequency of $1.46 \mathrm{kHz}$. When the laser power was increased it was necessary to make fine adjustments in the control parameters. Since the PI controller only allowed incremental adjustments in gain, these adjustments were accomplished by varying the cutoff frequency of the high pass filter which regulated the input signal amplitude.

Using the control parameters acquired, the laser wavelength was controlled to maintain the maximum resonant condition in the cavity. This behavior is shown in Figure 4.8 where both the reflected and transmitted signals from the cavity are shown. In Figure 4.8 the cavity was initially off resonance (region A) and then the cavity length was manually adjusted until the cavity was near resonance (point B). At this point, the feedback control 


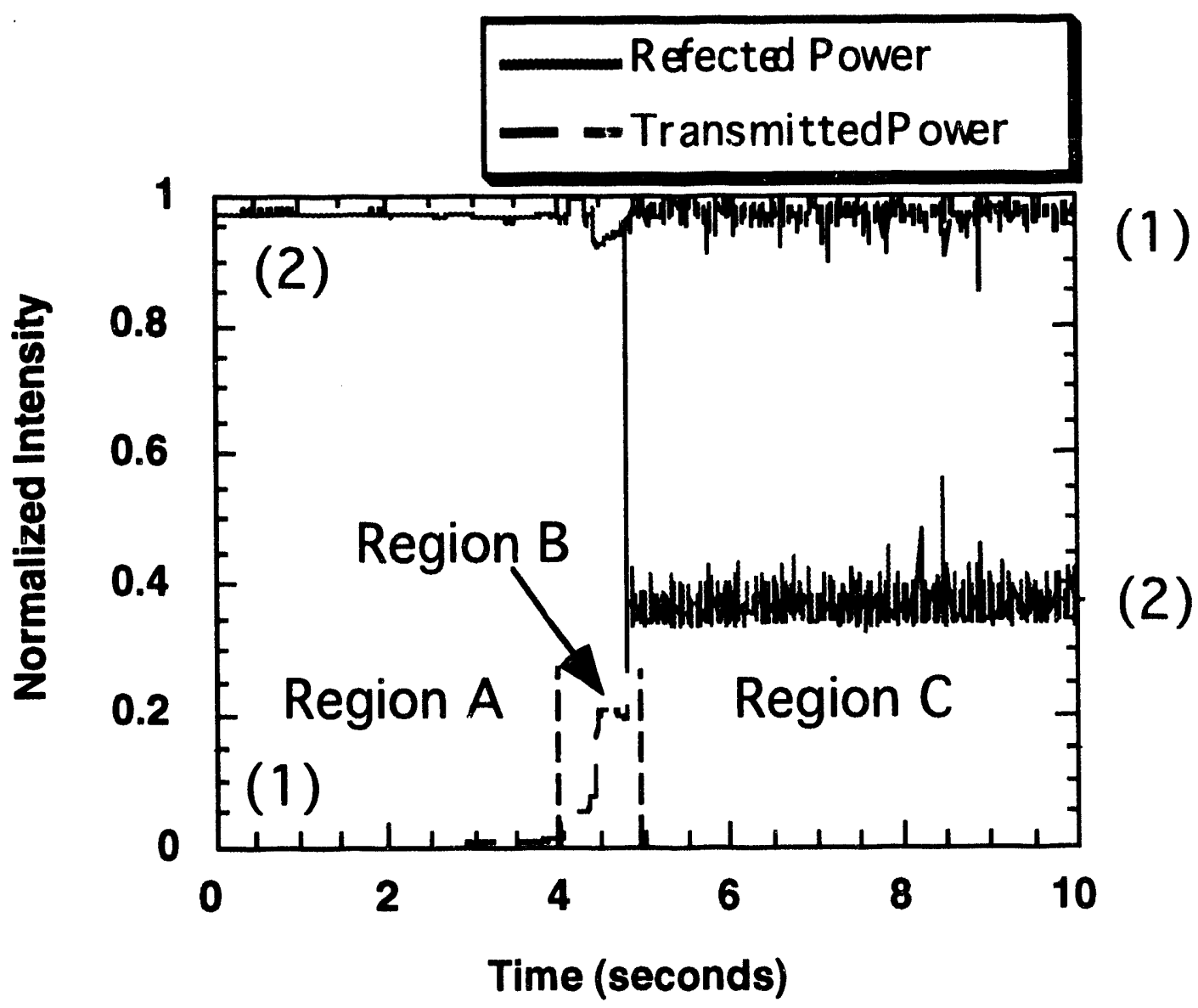

Figure 4.8 Graph showing measured transmitted (1) and reflected (2) signals for frequency-locking of laser diode to cavity resonance. 
loop began to respond to the detected error signal and frequency-locked the laser to the cavity (region $\mathrm{C}$ ).

Statistical analysis of the signal acquired during frequency-locking (see Fig. 4.9) was performed and the results are given in Table 4.4. The transmission data were acquired for a period of 6.7 minutes and 4000 data points were captured. Statistical analysis was performed on the data acquired to obtain a maximum value for the measured transmission intensity. The transmission data set was then normalized to this maximum value and again statistical analysis was performed. The results of the statistical analysis for this normalized data set are given in Table 4.4. As shown in Fig. 4.9, there is a relatively large swing in the mirimum and maximum values although the standard deviation is only 0.041 . The RMS value was less than $1 \%$ of the mean value obtained; however, the RMS value is 0.929 times the maximum transmission. These results indicate that the locking behavior of the laser to the cavity is relatively well behaved, although improvements could be made to maximize the power coupling to the cavity.

This method of frequency-locking the laser to the cavity allowed the cavity to be maintained on resonance for several minutes at a time with a moderate amount of noise in the laboratory and for longer periods of time when the lab was quiet. In addition, a 200 $\mathrm{Hz}$ vibration frequency was detected when the cavity feedback control was initially established; however, increasing the modulation frequency to $11 \mathrm{kHz}$ allowed the feedback control system to eliminate the effect of this low frequency noise. The system was determined to be somewhat sensitive to air currents caused primarily by the laboratory heating and cooling duct, which unfortunately was located almost directly above the experimental setup. The cavity locking was also sensitive to high frequency vibrations such as those caused by a sharp blow to the optical table surface. It was also observed that the performance of the feedback control improved as the cavity bandwidth was increased, e.g. when the losses to the SH been increased. 


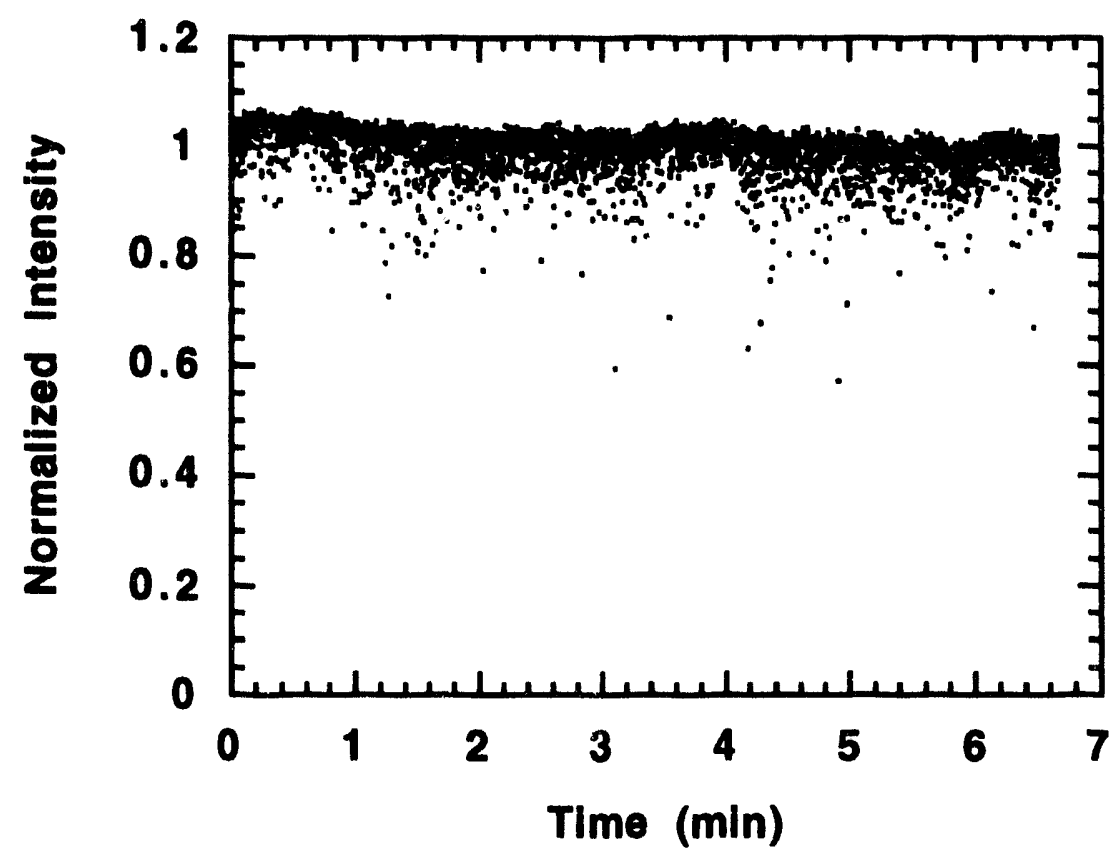

Figure 4.9 Measured transmission characteristics from the pumped-cavity while frequency-locking to the cavity was maintained using feedback control.

\begin{tabular}{|c|c|}
\hline Statistical Parameter & Statistical Value \\
\hline Minimum & 0.53476 \\
\hline Maximum & 1.00000 \\
\hline Mean & 0.92789 \\
\hline RMS & 0.92881 \\
\hline Standard Deviation & 0.04130 \\
\hline
\end{tabular}

Table 4.4 Statistical analysis of measured transmitted signal from the cavity when the laser was frequency-locked to the cavity. 
To illustrate the error signal obtained from this system, the cavity was purposely detuned to be slightly off resonance. The signals from the cavity transmission and the error signal were captured. These signals are shown in Figure 4.10. Note that the error signal amplitude is amplified 100 times. Also note that the large amplitude variations in the cavity transmission signal are due to the cavity being tuned slightly off resonance and that the relatively large negative going spikes each contain only a few data points out of 4000. A photograph of the instrumentation used in the frequency locking scheme is shown in Figure 4.11.

\subsection{Single Pass Second Harmonic Generation}

Power measurements obtained for the fundamental and $\mathrm{SH}$ wavelengths for the single pass SHG experiment are given in Table 4.5 (see Fig. 3.10 for the particular location of each of these power measurements). Taking the ratio of the total output power from the crystal, PIR+blue, to the infrared power out of the prism, $\mathrm{PIR}_{\mathrm{R}}$ a calibration factor was derived. This calibration factor was used to account for optical losses between the NLO crystal output and the prism output. It was assumed that, since the blue output from the prism was small, this value could be ignored. From these values, an optical loss coefficient of 0.13 was calculated. Using this optical loss coefficient to calibrate the power measurements out of the prism, a single pass conversion efficiency of

$$
\eta=0.0014
$$

was obtained. As expected, the SHG conversion efficiency was very small for this low intensity input beam.

Using it, measured values of the fundamental and SH powers, as well as the measured beam waist values, the nonlinear optical coefficient of the $3 \times 3 \times 5 \mathrm{~mm} \mathrm{KNbO}$ crystal was 


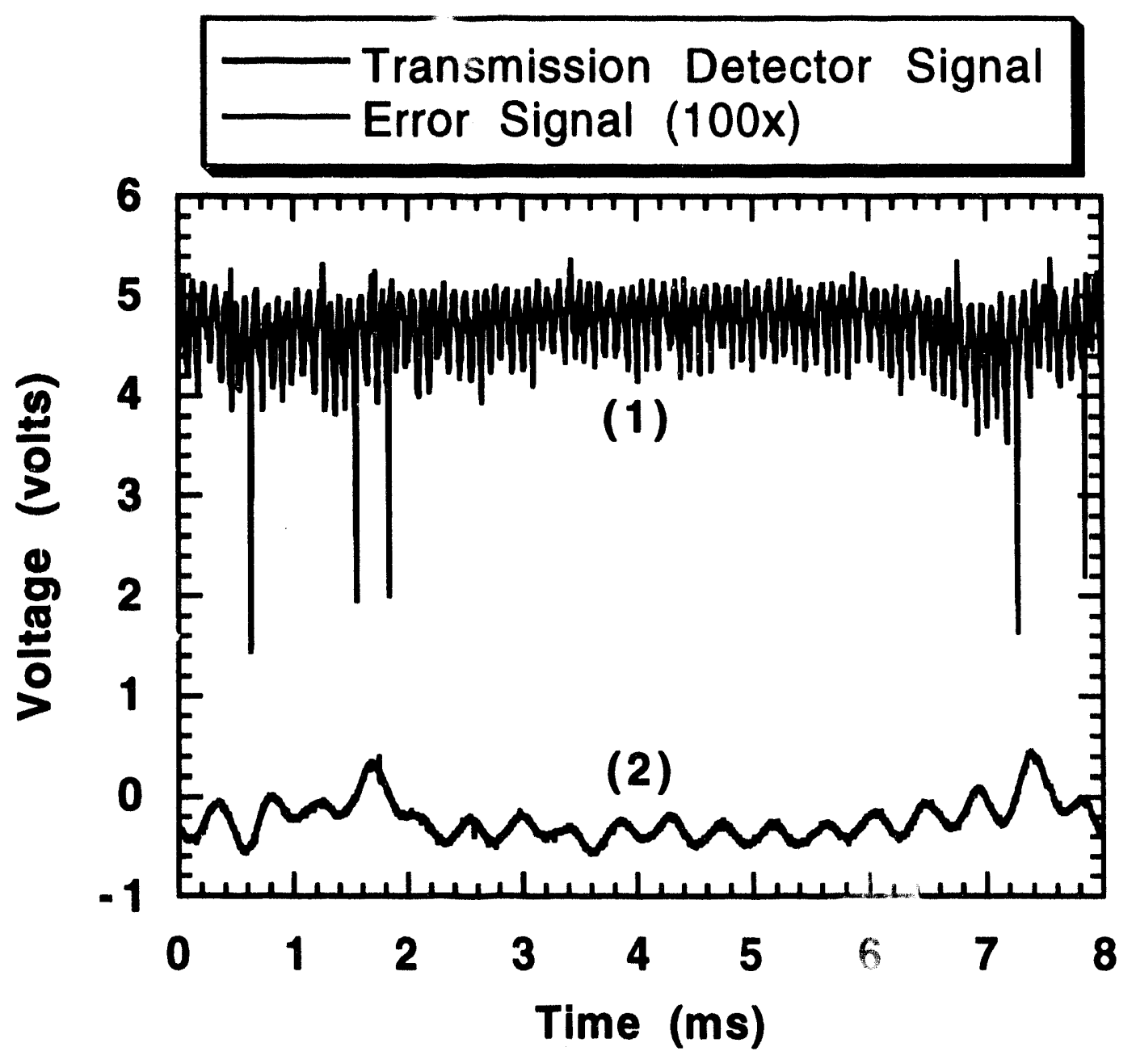

Figure 4.10 Graph showing measured transmission signal from cavity (1) when the cavity is detuned to be slightly off resonance and the corresponding error signal (2). 


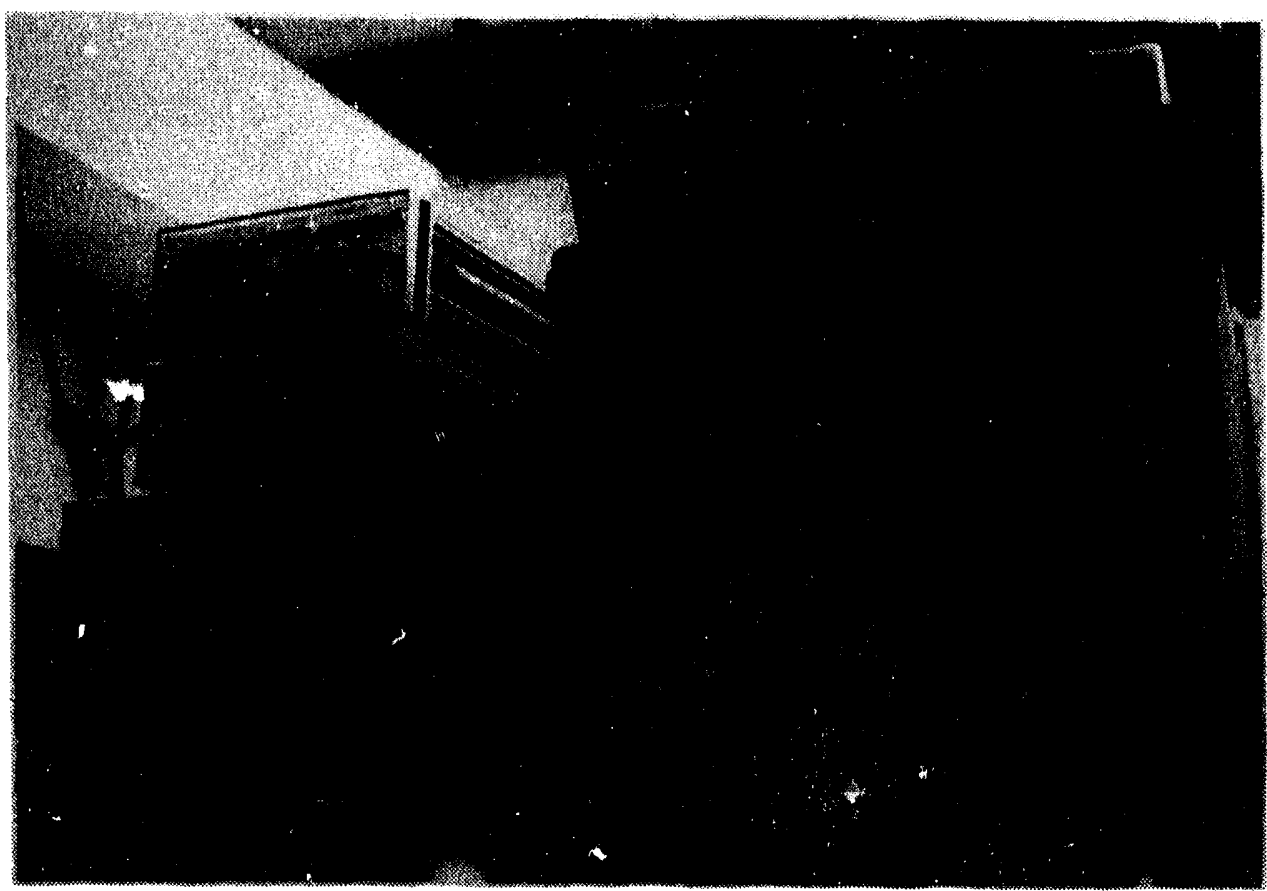

Figure 4.11 Photograph of equipment used in the frequency-locking the diode laser to the pumped-cavity.

\begin{tabular}{|c|c|}
\hline Power Measurement Variable & Power (mW) \\
\hline PIR $_{\mathrm{R}}$ & 82.9 \\
\hline PIR+blue $_{\text {IRorism }}$ & 81.8 \\
\hline P $_{\text {I }}$ & 72.4 \\
\hline P $_{\text {blueprism }}$ & 0.101 \\
\hline
\end{tabular}

Table 4.5 Measured beam powers obtained from the single pass SHG experiment. 
calculated. For this calculation, Eq. (2.29) was used and $\Delta k$ was assumed to be zero. This calculation led to a value of

$$
d=18.1 \mathrm{pm} / \mathrm{V} \text {. }
$$

This calculated value agrees well with the previously reported value ${ }^{[29]}$ of

$$
d=18.3 \mathrm{pm} / \mathrm{V} \text {. }
$$

Differential error analysis, performed to determine the uncertainty associated with this measurement, yielded an $8 \%$ uncertainty. Thus, these values are in agreement within the predicted uncertainty. In addition, the focus condition used in this experiment gave a beam waist length which was almost twice the crystal length. For optimum conversion efficiency, a reasonable limit $[3]$ is to have the beam waist length equal to the NLO crystal length. Thus, a small improvement in conversion efficiency would be expected by reducing the beam waist so that the waist length is equal to the crystal length.

\subsection{Pumped-Cavity Second Harmonic Generation}

Photographs of the experimental configuration are shown in Fig. 4.12. The complete experiment layout (see Fig. 4.12 a) shows the location of the various cptical elements used. Figure $4.12 \mathrm{~b}$ shows the optics used to circularize the diode laser output and to isolate the diode optically from the cavity. Figure $4.12 \mathrm{c}$ shows the actual cavity used in these experiments, with the NLO crystal located in the cavity. Finally, Fig. $4.12 \mathrm{~d}$ shows

the location of the various components used to separate and measure the $\mathrm{SH}$ and fundamental beams from the cavity.

After careful alignment of the cavity and placement of the NLO crystal within the cavity, the pumped-cavity SHG experiments were performed. The power reflection coefficient for the dichroic mirror used to separate the fundamental and SHG wavelengths from the cavity was determined to be 0.0058 at the laser fundamental wavelength. 
(a)

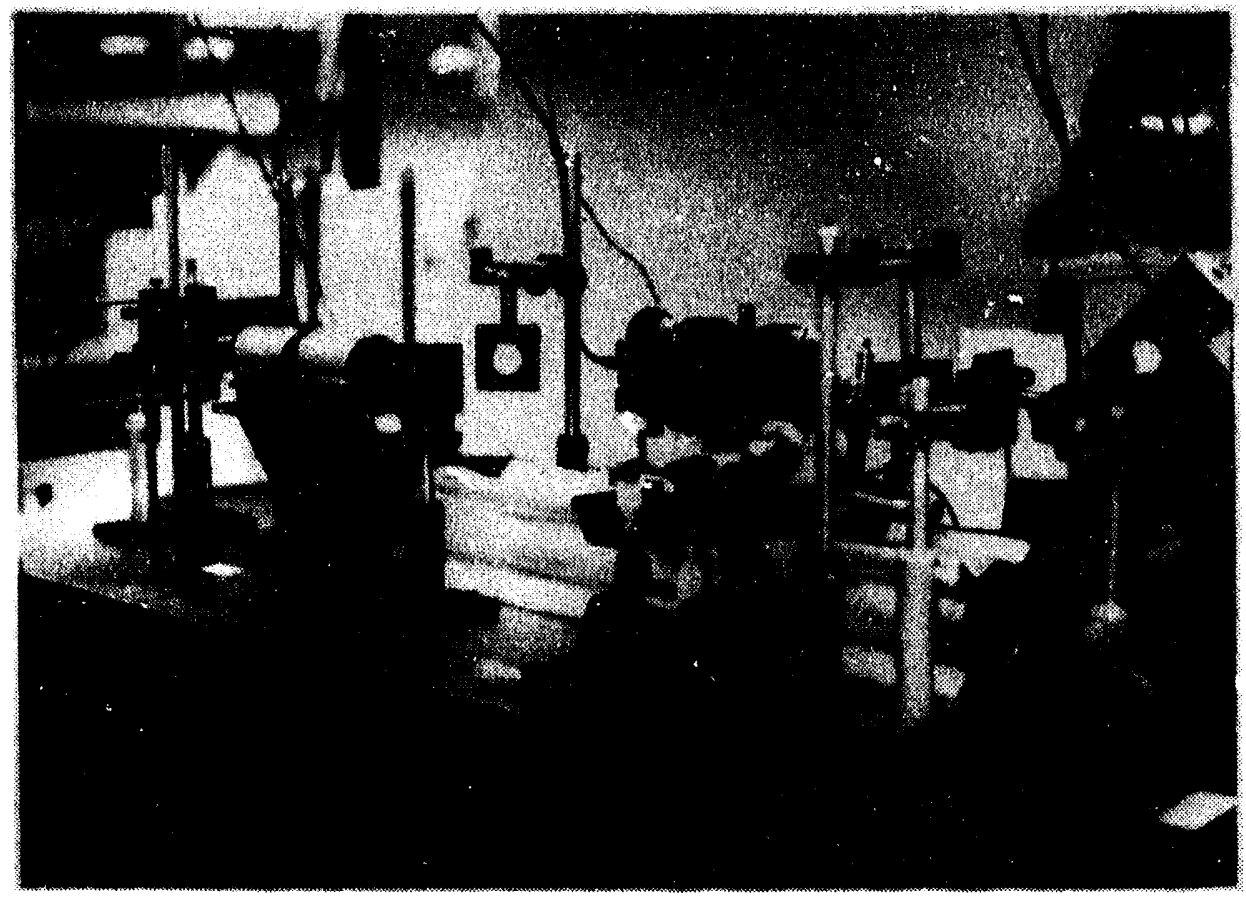

(b)

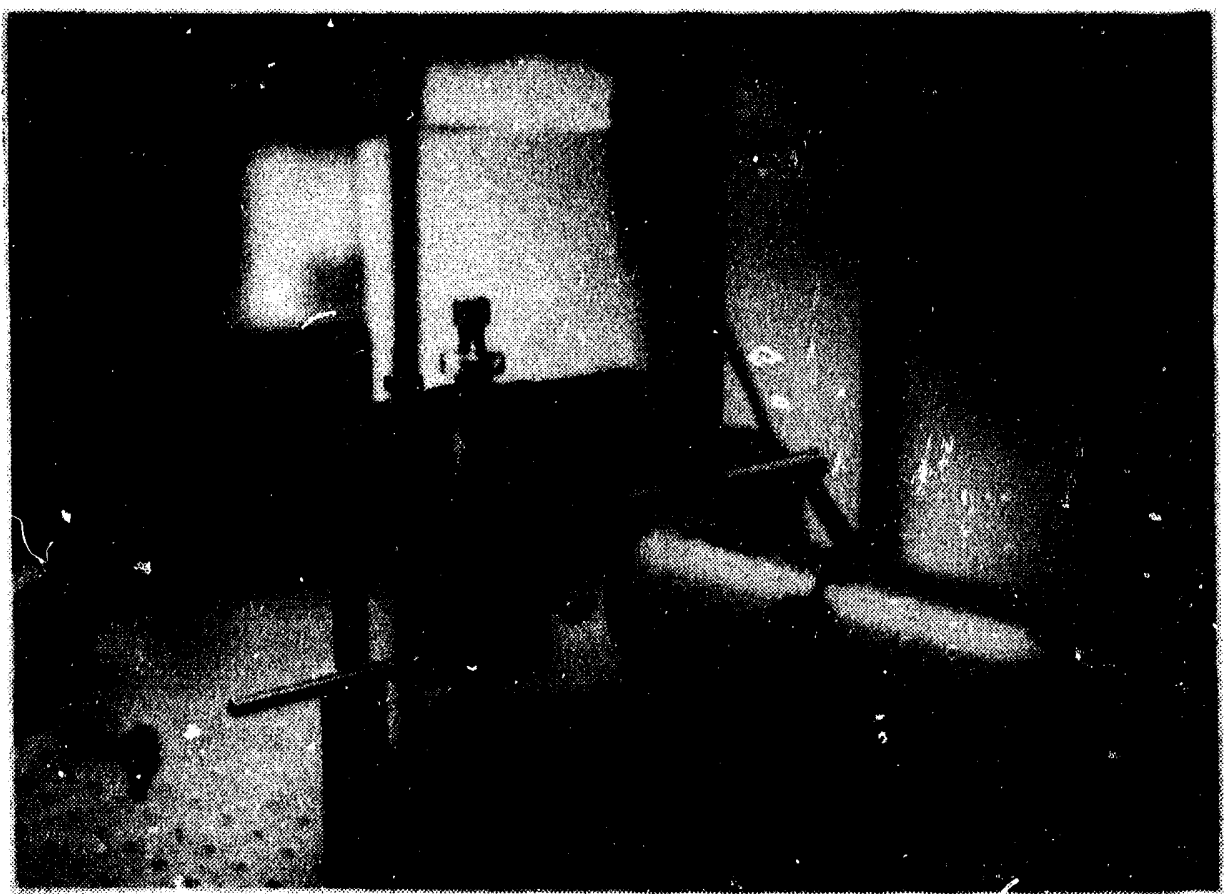

Figure 4.12 Photographs showing pumped-cavity SHG experiment configuration (a) overview of experiment showing general layout, (b) laser beam manipulation optics (c) pumped-cavity with NLO crystal holder, (d) optics used to separate the fundamental and $\mathrm{SH}$ wavelengths for power measurements. 

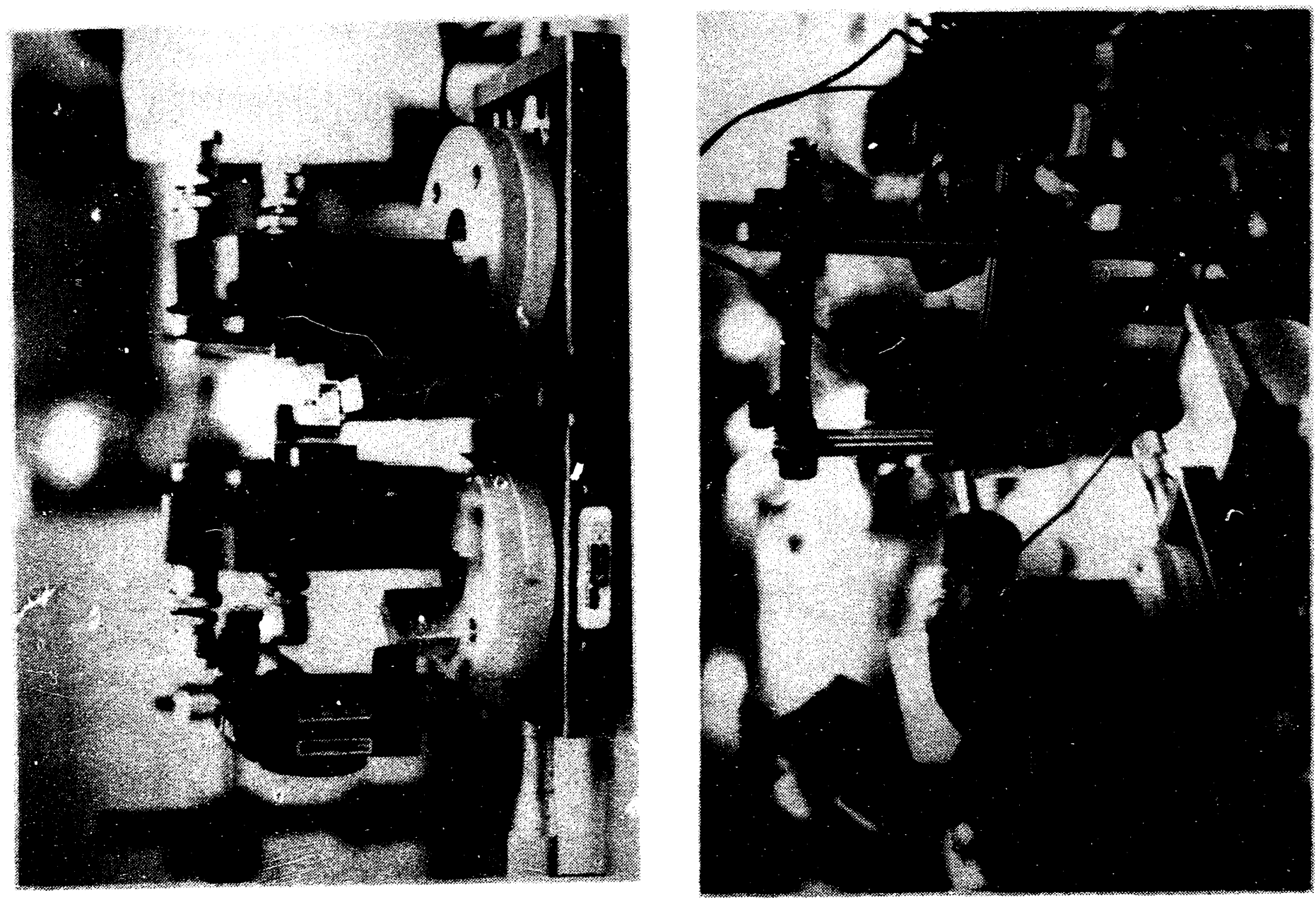

อ

ฮิ

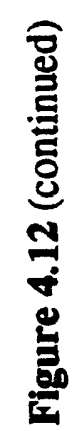


Since the power of the fundamental wavelength transmitted from cavity was on the order of $1 \mathrm{~mW}$, it was concluded that the power of the fundamental wavelength at the detector could be assumed to be negligible with respect to the measured SHG power. Although the dicde laser used in our application has a maximum rated output power of $150 \mathrm{~mW}$, due to losses associated with the various optical components used, only $50 \%$ of the laser power reached the cavity. However, even with this reduced power incident upon the cavity, efficient conversion to the second harmonic was achieved.

The linear cavity losses were obtained by measuring the cavity finesse when the crystal was detuned to prevent SHG. Substitution of the measured finesse value into Eq. (2.20) permitted the cavity loss parameter to be determined. Subsequent substitution of the calculated cavity loss parameter, along with the front and rear mirror reflectivity values, into Eq. (2.19) gave a single-pass linear loss value of 0.025 within the cavity, so that the single-pass cavity transmission was determined to be 0.975 . This value was subsequently used to determine the normalized cavity reflectance which would be expected for the measured experimental SH results.

The best results obtained are shown in Figure 4.13. The results given are the measured unidirectional SH output from the cavity and do not assume that the same amount of SHG occurs in the other direction as theory would predict 18,19$]$. Also, the loss of the SH light due to the $14 \%$ reflectivity of the output mirror ${ }^{[26]}$ is not included in these measurements. For these results, the unidirectional conversion efficiency obtained is $13 \%$ at the highest power. Assuming a $30 \%$ overall efficiency for the laser diode[20], this conversion efficiency would result in $7.8 \%$ overall efficiency for the $\mathrm{SH}$ source (assuming that the same SH power is produced in both directions within the cavity). This is a substantial improvement from the less than $0.1 \%$ efficiency for the argon ion laser. The measured values for the $\mathrm{SH}$ power out of the cavity were used to compare the experimental resuits 


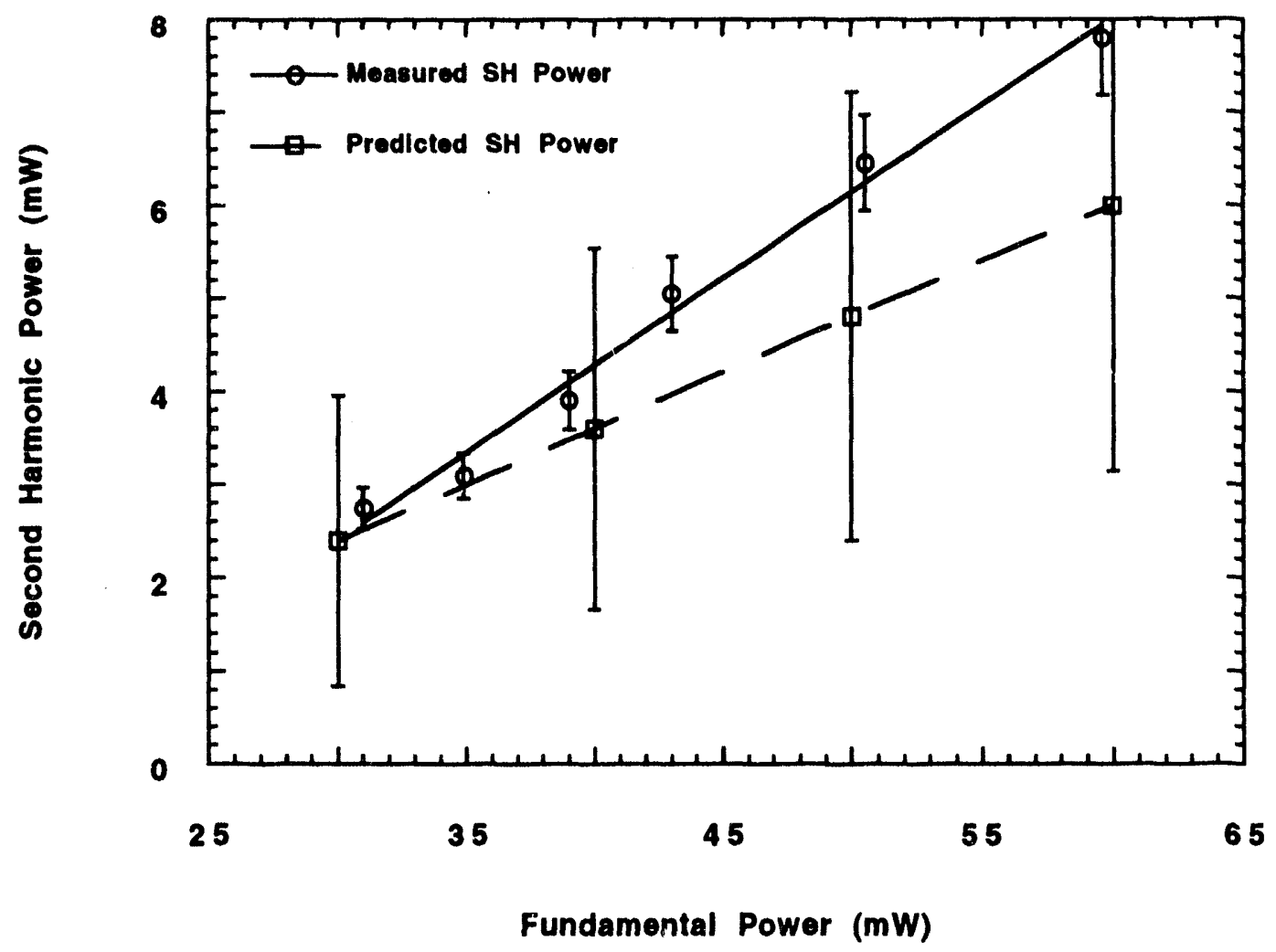

Figure 4.13 Measured unidirectional SH output power from pumped-cavity SHG experiments shown with predicted $\mathrm{SH}$ output power expected from the cavity. Differential error analysis was performed on the calculated values to generate error. 
with the expected thenretical results*. In addition, differential error analysis was performed on the relationships used to generate the predicted $\mathrm{SH}$ output power from the cavity. This analysis used a $0.5 \%$ error for the mirror reflectivities ${ }^{[26]}$, an error of 10 for the finesse measurements, an error of $8 \%$ for the input power values and an error of $1.1 \%$ was assumed for the nonlinear conversion factor $\gamma_{\mathrm{SH}}$ (due to variations between measured and reported values for the nonlinear optical coefficient of $\mathrm{KNbO}_{3}$ ). The error analysis, predicted for one $\sigma$, shows that these calculations are very sensitive to these relatively small errors. The results of this error analysis are included as error bars for the predicted $\mathrm{SH}$ values in Figure 4.13. This analysis also shows that our measured values agree with theory within the predicted error. The error bars included on the measured SH output values correspond the error associated with the calibrated detector which was determined from calibration measurements using differential error analysis.

Using the measured SHG values, it was determined that an enhancement of 13-14 in the circulating power within the cavity with respect to the incident power was obtained. When the conversion efficiency of the process is considered with respect to the circulating power within the cavity, it can be seen that the single-pass conversion within the cavity is approximately $1 \%$. This indicates, as was assumed in the model[7], that the nondepleted source approximation for the SHG process is a valid assumption.

From the measured SHG values, we calculated a cavity reflectance parameter which varied from 0.938 to 0.930 as the input power to the cavity was increased. These values for the cavity reflectance parameter indicate that the cavity was not perfectly impedancematched, so that improvements in the pumped-cavity performance would be expected by reducing the input mirror reflectivity. Calculations (see Appendix C) indicate that

\footnotetext{
* The results of the calculations used in comparing the experimental results to theoretical results is
} included in Appendix C. 
reducing the input coupler reflectivity to $94.44 \%$ would provide an enhancement of 1.65 in the output SH for the greatest input power condition.

The power reflected from the cavity was also measured during these experiments. These measurements were made with and without the cavity on resonance so that a normalized power reflectance could be obtained. The measured values for the normalized cavity reflectance were all between 0.45 and 0.50 . Substitution of the calculated cavity reflectance parameters into Eq. (2.39) predicted that the normalized power reflectance from the cavity should vary from 0.235 at the lowest input power to 0.28 at the highest input power. This gave a variation between theory and experiment of approximately 0.20 . This variation between the expected theoretical value and the measured value could in part be due to incomplete mode-matching. For the final cavity configuration, the mode-matching was not as good as that shown in Figure 4.5. This higher than expected reflectance from the cavity could also be due to the frequency-locking scheme since the RMS value of the transmitted light from the cavity had a normalized value of 0.929 . The effect of varying the cavity length would serve to $i$-srease interference within the cavity and thus increase the cavity reflectance somewhat. Finally, correcting the SHG power outputs to account for the output mirror reflectivity at the $\mathrm{SH}$ wavelength would further allow for a small increase in the cavity reflectivity.

It should be noted here that although the conversion efficiencies obtained here were relatively good for such low power laser light, that the results were significantly less than those predicted from theory (see Appendix A). The large linear losses associated with our cavity configuration proved to be the limiting factor in obtaining very high conversion efficiency. The results obtained using the monolithic ring cavity configuration ${ }^{[2]}$ implies that those cavity configurations are probably better for this application. However, care must be taken during manufacturing to insure that these cavities have good optical properties [30]. It should also be noted that the $\mathrm{SH}$ power 
output from the cavity does not follow the normal power-squared relationship since the circulating power within the cavity is nonlinear with varied cavity losses.

Beam radius measurements for the focused SH laser beam output from the cavity are shown in Figure 4.14. These results are given for two orthogonal directions corresponding to the planes parallel and normal to the diode junction. From these beam waist measurements, the SH laser beam quality was determined for various output powers and the results are given in table 4.6. As shown, the beam quality is approximately diffraction limited in the y-direction while the beam quality is 1.3-1.4 times the diffraction limit for the beam in the $\mathrm{x}$-direction. The $\mathrm{M}^{2}$ values obtained for the fundamental beam are 1.37 and 1.51 for the $x$ - and $y$-directions respectively. Comparing these values with the values for the SH harmonic beam shows that the beam quality in the $\mathrm{x}$-direction remained approximately constant while the beam quality in the $\mathrm{y}$-direction has improved to be near diffraction-limited. The fact that the beam quality has remained constant in the $x$-plane could be due to a slight misalignment of the cavity in the $x$ direction, i.e. the cavity is a slightly off-axis confocal cavity. This would also account for the increased reflection from the cavity.

Next the phase-matching temperature bandwidth of the $\mathrm{KNbO}_{3}$ crystal was determined experimentally. The results from the measurements are shown in Figure 4.15, indicating that the phase-matching temperature band shifts to higher temperature with increased laser power. Since the laser wavelength also increases with power ${ }^{[18]}$, this indicates that the phase-matching temperature increases with increasing wavelength. This is consistent with reported results for SHG phase-matching in $\mathrm{KNbO}_{3}{ }^{[31]}$.

The FWHM temperature bandwidth was determined graphically from the measured points. These results are given in Table 4.7. The measured bandwidths for the various 

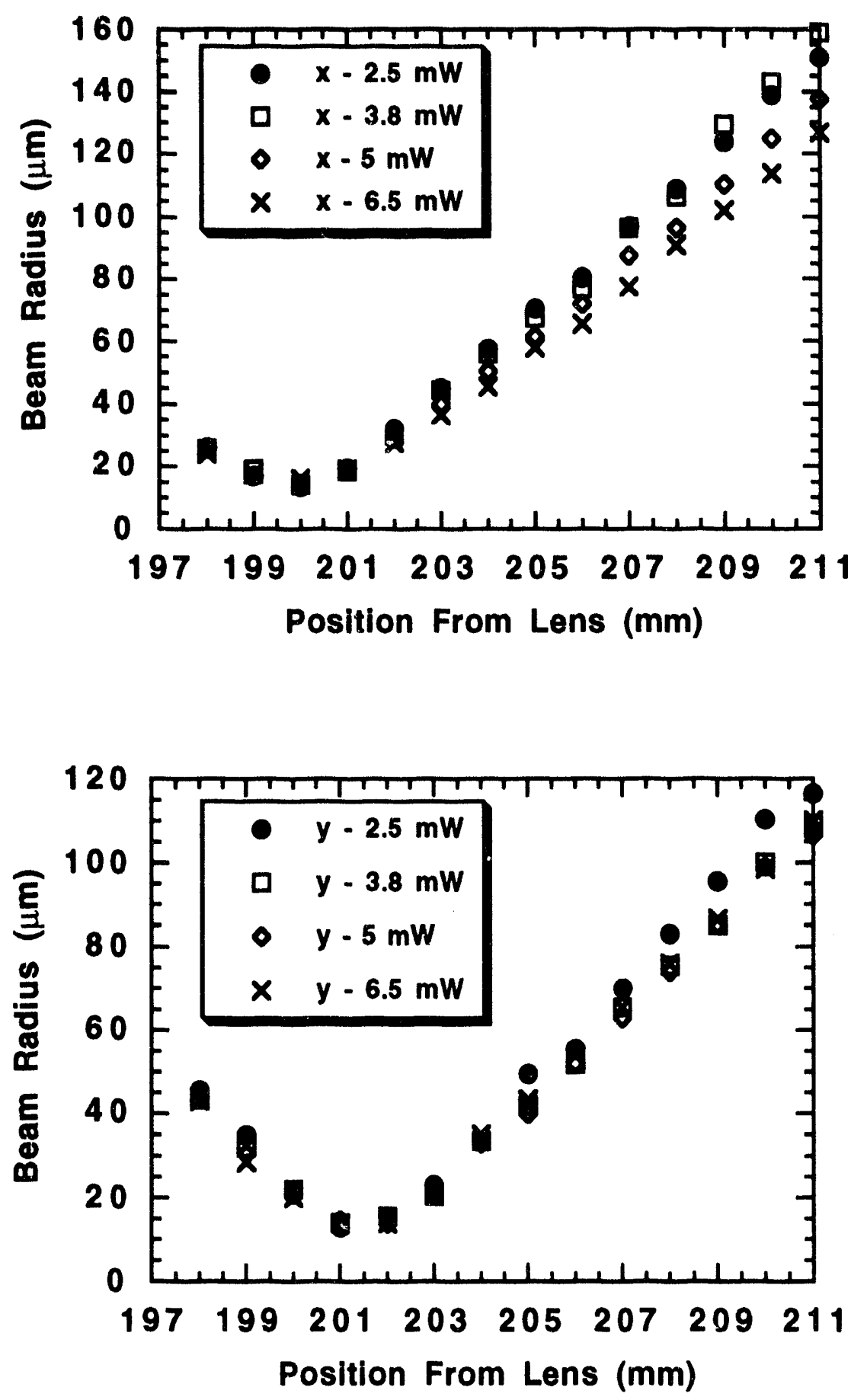

Figure 4.14 Measured beam radii for the SH beam output from the pumped-cavity. 


\begin{tabular}{|c|c|c|}
\hline SH Power (mW) & $M_{x}^{2}$ & $M_{y}^{2}$ \\
\hline 2.5 & 1.366 & .997 \\
\hline 3.8 & 1.35 & 1.013 \\
\hline 5.0 & 1.266 & 1.279 \\
\hline 6.5 & 1.303 & 1.054 \\
\hline
\end{tabular}

Table 4.6 Calculated beam quality for SH laser light output from pumped-cavity experiment.

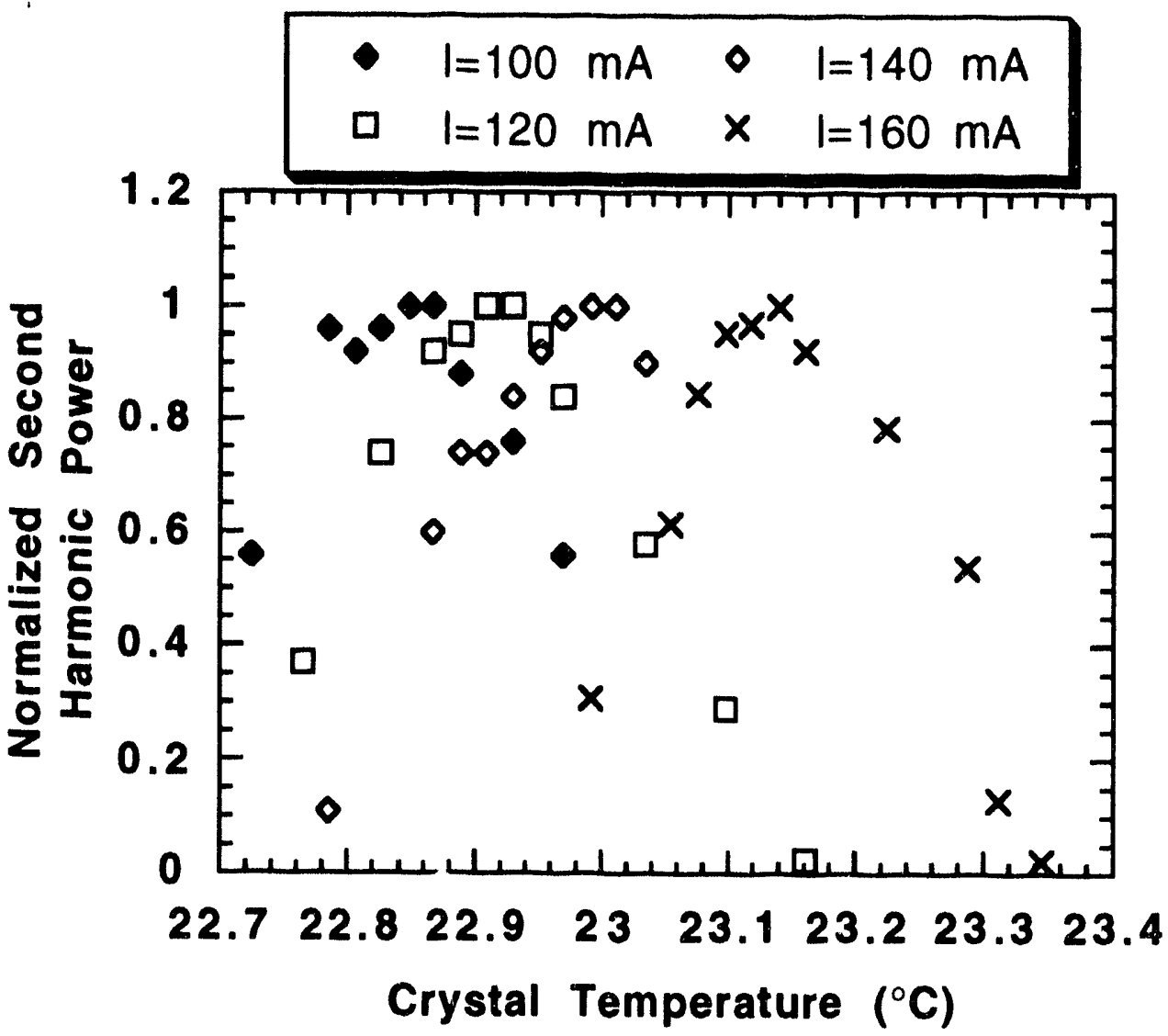

Figure 4.15 Measured SHG at various power levels and temperatures used to determine the phase-matching temperature bandwidth. 
powers used range from $0.26-0.28^{\circ} \mathrm{C}$. It should be noted that the measured bandwidths correspond to the SHG at 2.5, 3.8 and $6.5 \mathrm{~mW}$. Although measurements were made for SHG at $5 \mathrm{~mW}$, there was an insufficient number of points to determine the bandwidth at this power level. However, it is expected that the phase-matching temperature bandwidth at this power level will be consistent with the other measured values. In addition, the values obtained for the FWHM phase-matching temperature are consistent with previously reported results $[31]$.

\subsection{Summary}

In this chapter, the results from the experiments leading up to, and including, the pumped-cavity SHG experiment are given. The results from the laser beam characterization show that the laser linewidth is compatible with the cavities used in these experiments. In addition, the apparent beam concept allows very good mode-matching to the cavities to be achieved.

Results obtained show that the frequency locking system works well and that, when the cavity length is brought near resonance, the feedback control loop locks the laser frequency to the cavity frequency. Statistical analysis was performed on the cavity transmission to characterize the performance of the system. The results indicate that although the locking system works well, some minor improvements could be made to optimize its performance.

The results from the single-pass SHG experiment were presented and the value obtained for the nonlinear optical coefficient of the $\mathrm{KNbO}_{3}$ crystal are in good agreement with previously published values. 


\begin{tabular}{|c|c|}
\hline Second Harmonic Output Power $(\mathrm{mW})$ & Phase-Matching Temp. Bandwidth $\left({ }^{\circ} \mathrm{C}\right)$ \\
\hline 2.5 & 0.26 \\
\hline 3.8 & 0.26 \\
\hline 5.0 & - \\
\hline 6.5 & 0.28 \\
\hline
\end{tabular}

Table 4.7 Phase-matching temperature bandwidth determined graphically from experimental results. 
Finally, the results from the pumped-cavity SHG experiments are given. These results show that the cavity performance is limited due to the relatively large linear losses associated with the cavity. However, even with these larger than expected losses, the overall conversion efficiency from the fundamental wavelength to the $\mathrm{SH}$ wavelength is very good. In addition, the SH beam quality is comparable to the fundamental pump beam quality and the overall electrical to light conversion for the blue wavelength is two orders of magnitude greater than that of a typical argon lasers. 


\section{CHAPTER 5}

\section{Summary and Conclusions}

In these experiments, we have demonstrated the feasibility of using pumped-cavity SHG to produce a visible wavelength laser source possessing an electrical to light conversion efficiency of $7.8 \%$. Differential error analysis was performed to determine the error asscciated with theoretically predicted results obtained using a model. Comparison between the measured experimental results and the predicted theoretical results show agreement within the calculated error. This work has provided support for the model used in designing the pumped-cavity SHG experiments and provides insightful information for those wishing to investigate this process. In addition, we have demonstrated that the beam quality associated with the output SH beam from the cavity is near-diffraction limited thus possessing optimum focusing properties.

Before attempting to perform the pumped-cavity SHG experiment, several of the key areas included in the experiment were investigated to develop a fundamental understanding of each of these areas individually. These steps were necessary to insure optimal results from the pumped-cavity SHG process. These key areas included: laser beam manipulation, laser beam characterization, Fabry-Perot cavity characterization, frequency-locking of the laser to the cavity and single pass SHG.

Standard techniques were used to collimate, circularize and optically isolate the diode laser from the cavity. After the laser beam was modified, a technique was used to locate an apparent beam waist in space. Using this apparent beam waist approach allowed the beam, due to the diode laser, to be characterized while ignoring the effects of the optical elements used prior to a waist creating lens. The identified apparent beam waist was used to mode-match the diode laser beam to the various cavities used in these experiments. As 
experimental results show, this approach allows precise mode-matching to the cavity to be obtained. The effects of impedance-matching to a cavity were also investigated. By varying the losses within the cavity it was shown that the reflectance from the cavity was reduced to account for the higher losses within the cavity.

To lock the laser wavelength to the cavity resonance peak, a dithering technique was used. The dithering technique allowed the laser wavelength to be maintained on the cavity resonance peak with a RMS transmission value of 0.93 times the peak detected transmitted signal for a period of 6.7 minutes. Thus, the locking technique provided a stable cavity output for extended periods of time. In addition, the stability improved for shorter periods of time and the dithering technique allowed the cavity locking to be stable with a $200 \mathrm{~Hz}$ background vibration.

A single-pass SHG experiment was performed to measure the nonlinear optical coefficient for one of the $\mathrm{KNbO}_{3}$ crystals used in these experiments. The measured value of $18 \mathrm{pm} / \mathrm{V}$ was in good agreement with previously reported values and was used in all subsequent calculations. The results obtained from the pumped-cavity SHG experiment differed significantly from those predicted from the original design parameters. This was primarily due to large linear losses encountered with the mirrors and the NLO crystal used in the experiment. In the original calculations, a single-pass linear loss within the cavity was assumed to 0.004 while the measured single-pass linear loss within the cavity was 0.025 . This effected the experiment in two ways: (1) the cavity input mirror was not impedance-matched, thus the power coupling to the cavity was reduced and (2) the large linear losses reduced the circulating power enhancement within the cavity further impeding the SHG process. These problems can be overcome by selcting an input mirror reflectivity which better matches the cavity reflectane parameter and by using higher quality optics. 
The expected reflectance from the cavity was calculated using the measured SH output power values. These values were then compared to those values measured for the reflected power from the cavity. These comparisons indicated that the reflectance from the cavity was approximately $20 \%$ greater than would be expected. It is believed that this additional reflectance was due to the less than optimum RMS value for the transmission from the cavity as well as some residual mode-matching error.

Calculations also indicated that replacing the cavity input mirror with a mirror with a reflectivity of 0.944 will almost double the SH output power from the cavity. This experiment will be performed as part of the ongoing pumped-cavity SHG experiment. In addition, a configuration will be used which will allow the SH power generated in two directions to be measured.

In continuation of this work, the feasibility of using phase-locked diode laser arrays as pump sources for the cavity will be investigated. A $0.5 \mathrm{~W}$ diode array will be used initially in these experiments. Additionally, the feasibility of using master-oscillator power-amplifier (MOPA) semiconductor lasers as pump sources to produce very high pcwer outputs from the pumped-cavity experiment will be investigated. These devices are capable of output powers $>1 \mathrm{~W}$ in a single transverse and single axial mode and thus could provide an alternative source to argon-ion lasers. Finally, a monolithic cavity will be designed to provide low intracavity losses to obtain very high conversion efficiency to visible wavelengths, and alternative methods to frequency-lock the laser to the cavity will be explored. 


\section{APPENDIX A}

\section{Calculations for Pumped-cavity Second Harmonic Generation of $860 \mathrm{~nm}$ Diode Laser}

These appendicies contain MATHCAD files which were used to design the pumpedcavity SHG experiment and to evaluate the results obtained. To find the input mirror reflectivity value required to impedance-match the cavity, several parameters must first be evaluated. The first parameter to be solved for is the nonlinear conversion factor $\gamma_{\mathrm{SH}}$, which is determined using the Boyd and Kleinman focusing factor [18]. First, the variables to be used in these calculations will be defined.

$$
\begin{array}{lllll}
\mathrm{B}:=10^{-9} & 1:=0.005 & \lambda:=860 \cdot 10^{-9} & w_{0}:=59 \cdot 10^{-6} & \mathrm{n}:=2.23 \\
\mathrm{c}:=3 \cdot 10^{8} & \omega:=\frac{2 \cdot \pi \cdot \mathrm{c}}{\lambda} & \mathrm{k}_{\omega}:=\frac{2 \cdot \pi \cdot \mathrm{n}}{\lambda} & \varepsilon_{0}:=8.854 \cdot 10^{-12} & \mu:=4 \cdot \pi \cdot 10^{-7}
\end{array}
$$

For these parameters $\mathrm{B}$ is the double refraction parameter, which for noncritical $\left(90^{\circ}\right)$ phase-matching is zero, 1 is the NLO crystal length, $\lambda$ is the diode laser output fundamental wavelength, $w_{0}$ is the minimum beam waist within the cavity, $n$ is the NLO crystal index of refraction, $c$ is the speed of light in vacuum, $\omega$ is the light frequency in radians, $\mathbf{k}_{\omega}$ is the beam propagation factor within the NLO crystal, $\varepsilon_{0}$ is the dielectric permittivity of free space and $\mu$ is the magnetic permeability. Using these parameters, a dimensionless longitudinal coordinate parameter $\xi$ can be defined as

$$
\xi:=\frac{1 \cdot \lambda}{w_{0}{ }^{2} \cdot 2 \cdot \pi}
$$

which has a solution given by

$$
\xi=0.1966
$$


Using this value, then the Boyd and Kleinman focusing factor can solved for in terms of the double refraction constant B and the dimensionless longitudinal coordinate parameter $\xi$. For these calculations $\Delta \mathrm{k}$ is the wavevector mismatch parameter and is approximately equal to zero. For simplicity let

$$
\beta:=B \cdot \xi^{-\frac{1}{2}}
$$

and

$$
\sigma:=\frac{1}{2 \cdot \xi} \cdot \Delta k
$$

Then the Boyd and Kleinman focusing factor is given by

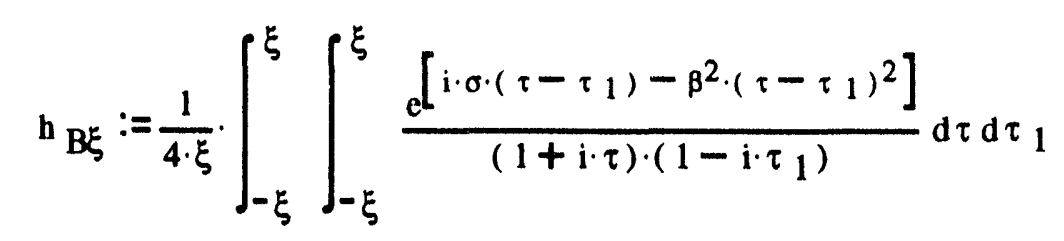

and solving this equation numerically leads to the solution given by

$$
\mathrm{h}_{\mathrm{B} \xi}=0.1917
$$

Defining the nonlinear optical coefficient of the $\mathrm{KNbO}_{3}$ crystal to be used in these experiments as

$$
d_{\text {eff }}:=18.1 \cdot 10^{-12}
$$

and using the Boyd and Kleinman focusing factor, the nonlinear conversion factor $\gamma_{\mathrm{SH}}$ can now be solved for. Given that

$$
\gamma_{S H}:=\frac{2 \cdot \omega^{2} \cdot d_{\mathrm{eff}^{2}} \cdot k \omega}{\pi \cdot n^{3} \cdot \varepsilon_{\mathrm{o}} \cdot c^{3}} \cdot \mathrm{l} \cdot \mathrm{h}_{\mathrm{B}} \xi
$$

then the solution to this equation is

$$
\gamma_{\text {SH }}=0.0059
$$


It is this factor which will be used to determine the conversion from the laser fundamental wavelength to the SH wavelength. This value can now be used to determine the cavity reflectance parameter based on assumed cavity losses.

The cavity reflectance parameter $r_{m}$ which accounts for the round-trip losses within a resonant cavity is given by

$$
r_{m}=t^{2} \cdot t_{S H}^{2} \cdot r_{2}
$$

where $r_{2}$ is the cavity output mirror power reflection coefficient and $t_{S H}$ is the cavity nonlinear transmission factor which account for losses from the fundamental wavelength to the SH wavelength. The nonlinear transmission factor is given by

$$
{ }^{t} S_{H}=\left(1-\gamma_{S H} \cdot P_{c}\right)
$$

where $P_{c}$ is the circulating beam power within the cavity. Then the power reflected from the resonant cavity is given by

$$
P_{r}=\frac{\left(\sqrt{r_{1}}-\sqrt{r_{m}}\right)^{2} \cdot P_{i n}}{\left(1-\sqrt{r_{m} \cdot r_{1}}\right)^{2}}
$$

where $P_{i n}$ is the input beam power to the cavity and $r_{1}$ is the input mirror power reflection coefficient. The relationship describing the circulating power within the pumped-cavity is

$$
P_{c}=\frac{t_{1} \cdot P_{\text {in }}}{\left(1-\sqrt{r_{1} \cdot r_{m}}\right)^{2}}
$$

For maximum power coupling into the cavity it is necessary to reduce the cavity reflectance to zero. From Eq. (3) it can be seen that this condition will occur when the input mirror power reflection coefficient is equal to the cavity reflectance parameter. When this condition is satisfied, Eq. (4) reduces to

$$
P_{c}=\frac{P_{\text {in }}}{1-r_{m}}
$$


Substitution of Eq. (5) into Eq. (2) and then subsequent substitution of the resulting relationship for $\mathrm{t}_{\mathrm{SH}}$ intu $\mathrm{Eq}$. (1) then leads to a relationship in terms of known variable which can then be solved to determine the value of the cavity reflectance parameter for optimum power coupling into the cavity. This relationship is given by

$r_{m}{ }^{3}-\left(2+t^{2} \cdot r\right) \cdot r_{m}{ }^{2}+\left[1+2 \cdot t^{2} \cdot r_{2} \cdot\left(1-\gamma S_{H} \cdot P_{\text {in }}\right)\right] \cdot r_{m}-t^{2} \cdot r_{2} \cdot\left(1-\gamma S_{H} \cdot P_{i n}\right)^{2}=0$

Now the variables used to calculate the cavity reflectance parameter will be defined.

$\mathrm{r}_{2}:=.9996 \quad \mathrm{P}_{\text {in }}:=0.075 \quad \mathrm{t}:=0.996 \quad \alpha:=\mathrm{t}^{2} \cdot \mathrm{r}_{2} \quad \alpha:=\mathrm{t}^{2} \cdot \mathrm{r}_{2}$

MATHC. D's root solve function can now be used to solve for the relationship describing $r_{m}$ and determine the real value(s) where $r_{m}$ goes to zero. The initial guess value and the range over which $r_{m}$ will be solved for must be input.

$$
\begin{gathered}
r_{m}:=.901 . .1 \quad \text { TOL }:=0.0000000000001 \\
\operatorname{Reflect}\left(r_{m}\right)=r_{m}{ }^{3}-(2+\alpha) \cdot r_{m}{ }^{2}+\left(1+2 \cdot \alpha \cdot \beta_{1}\right) \cdot r_{m}-\alpha \cdot \beta_{1}^{2} \\
R_{m}\left(r_{m}\right):=\operatorname{root}\left(\operatorname{Reflect}\left(r_{m}\right), r_{m}\right)
\end{gathered}
$$

Solution to this relationship leads to a value of

$$
R_{m}\left(r_{m}\right)=0.966
$$

Thus to perfectly impedance match to the cavity, the input mirror power reflection coefficient $r_{1}$ must have a value of 0.966

Based on this input mirror reflectivity, the cavity performance can now be predicted. Modifying Eq. (5) to use the calculated cavity reflectance parameter, the circulating power within the cavity can be calculated as 


$$
\begin{gathered}
P_{c}\left(r_{m}\right):=\frac{P_{\text {in }}}{1-R_{m}\left(r_{m}\right)} \\
P_{c}\left(r_{m}\right)=2.2048
\end{gathered}
$$

This calculation shows that the circulating power within the cavity is $2.205 \mathrm{~W}$. Based on this circulating power, the expected SH output power from the cavity can now be predicted using

$$
\begin{gathered}
P_{S H}\left(r_{m}\right):=2 \cdot \gamma_{S H} \cdot P_{c}\left(r_{m}\right)^{2} \\
P_{S H}\left(r_{m}\right)=0.0574
\end{gathered}
$$

This calculation indicates that the expected SH power output from the cavity will be 27.4 $\mathrm{mW}$ which includes the total output power from both sides of the standing wave cavity. Next, the expected overall conversion efficiency can be determined from

$$
\begin{aligned}
\eta_{S H}\left(r_{m}\right) & :=\frac{P_{S H}{ }^{\left(r_{m}\right)}}{P_{\text {in }}} \\
\eta_{S H}\left(r_{m}\right) & =0.765
\end{aligned}
$$

This calculation shows that the expected conversion efficiency to the $\mathrm{SH}$ wavelength is $76.5 \%$. This is an extremely good conversion. To determine the validity of using the nondepleted source approximation used in the development of this model, the conversion efficiency of the circulating beam within the cavity will now be calculated. This is determined using

$$
\begin{gathered}
\eta_{\text {SHCavity }}\left(r_{m}\right):=\frac{P_{S_{H}}\left(r_{m}\right)}{P_{c}\left(r_{m}\right)} \\
\eta_{\text {SHCavity }}\left(r_{m}\right)=0.026
\end{gathered}
$$

and this calculation shows that the single-pass conversion of the beam within the cavity is $2.6 \%$. This result verifies that the nondepleted source approximation used in the model development is indeed valid. 


\section{APPENDIX B}

\section{Determination of Laser Beam Quality and Apparent Beam Parameters}

From Siegman, and usso from Sasnett and Johnston $[12,13]$, the relationships between a real multimode laser beam and a fundamental mode beam can be deternined by assuming an embedded Gaussian beam propagating within the multimode beam. In this geometry, the following relationships are defined:

$\mathrm{W}_{0}:=55.5 \cdot 10^{-6} \quad \mathrm{w}_{1}:=95.68 \cdot 10^{-6} \quad \mathrm{z}_{1}:=0.242 \quad \mathrm{z}_{0}:=0.2529 \quad \lambda:=860 \cdot 10^{-9}$

where $\mathrm{W}_{\mathrm{O}}$ is the measured radius of the real beam minimum waist at a location $\mathrm{z}_{\mathrm{O}}$ along the beam optical axis, $W_{1}$ is the measured radius of the reai beam at a position $z_{1}$ along the beam optical axis and $\lambda$ is the laser wavelength. Then using these parameters, various values for the real beam can be determined. The real beam half-angle divergence is given by

$$
\theta:=\sqrt{\frac{w_{1}{ }^{2}-w_{0}{ }^{2}}{\left(z_{1}-z_{0}\right)^{2}}}
$$

Using this value for the measured real beam half-angle divergence, a relationship can be defined which is indicative of the real beams deviation from the theoretical diffraction limited case. This factor is referred to as the real beam propagation factor and is given by

$$
M:=\sqrt{\frac{W_{0} \cdot \theta \cdot \pi}{\lambda}}
$$

It is the Rayleigh range which relates the real laser beam to the embedded Gaussian beam within the real beam. The Rayleigh range is the same for both of these beams and is defined as the distance along the beam optical axis where the beam radius has increased by a factor of $\sqrt{2}$ with respect to the minimum beam waist radius. Once the beam Rayleigh range has been determined, then this value can be used in calculating the 
embedded Gaussian beam radius at the minimum waist location $\mathrm{z}_{0}$. This minimum waist value is given by

$$
w_{0}:=\sqrt{\frac{z_{R} \cdot \lambda}{\pi}}
$$

After determination of the embedded Gaussian beam waist, an optical system can then be designed using Gaussian beam propagation theory and assuming propagation of the embedded Gaussian beam through the optical system. After propagation, the real beam waist will be located at the same position and possess the same wavefront radius of curvature as the embedded Gaussian beam. In addition, the real beam divergence and radius will be proportional to the embedded Gaussian beam parameters anywhere throughout the optical system and be related by the propagation factor $\mathbf{M}$.

In Klein and Furtak ${ }^{[11]}$, the relationships for Gaussian beam propagation through a lens are given. For a beam with known Gaussian parameters, these relationships allow the beam parameters on the transmission side of the lens to be predicted accurately. For our application, using a lens to create a beam waist in space allows the beam's Gaussian parameters to be determined experimentally. Then, using the measured beam Gaussian parameters, the theory for Gaussian beam propagation can be used to propagate the beam backwards through the waist-creating lens mathematically. This technique will allow relationships to be developed which describe an apparent beam waist radius, Rayleigh range and location in space on the laser side of the waist-creating lens. This apparent beam waist can then be used in developing mode-matching relationships to a cavity whose Gaussian beam parameters are known.

We will now use this concept to determine the apparent beam Gaussian parameters from the diode laser. A lens of focal length $f$ is used to create a beam minimum waist in space 
at a position $S$ with respect to the lens principal plane. We now define the lens focal length and the minimum waist location to be used in these calculations as

$$
f:=0.25 \quad S:=0.2529
$$

Using these variables, as well as the previously calculated values from the measured input beam parameters, an apparent beam waist location in space $S_{1}$ is determined from

$$
S_{1}:=f+\frac{t^{2} \cdot(S-f)}{(S-f)^{2}+z^{2}{ }^{2}}
$$

to be

$$
S_{1}=2.89
$$

Then the apparent beam Rayleigh range $\mathrm{z}_{01}$ given by

$$
z_{o l}:=\frac{z_{R} \cdot t^{2}}{(S-f)^{2}+z_{R}^{2}}
$$

is calculated to be

$$
\mathrm{z}_{\mathrm{ol}}=7.066
$$

From this value for the apparent beam Rayleigh range, the apparent beam waist $w_{0} 1$ given by

$$
w_{o l}:=\sqrt{\frac{z_{01} \cdot \lambda}{\pi}}
$$

is determined to be

$$
\mathrm{w}_{\mathrm{ol}}=0.00139
$$

The units for the above measured values are meters. Finally, the real beam propagation factor for this beam is determined to be

$$
M=1.204
$$

It is these values for the apparent beam which were used in developing mode-matching relationship to the pumped-cavity. 


\section{APPENDIX C}

\section{Comparison of Measured Experimental Results with Theory}

In this section, the experimentally obtained results will be used to develop relationships to determine the correlation between the experiment and theoretical results due to the Ashkin, Boyd and Dziedzic theory[7]. These results will be evaluated for the $2.5 \mathrm{~cm}$ cavity with a $7.5 \mathrm{~mm}$ long $\mathrm{KNbO}_{3} \mathrm{NLO}$ crystal in the cavity. It should be noted here that the results obtained in Appendix A were for the initial cavity design where a $5 \mathrm{~mm}$ long $\mathrm{KNbO}_{3}$ crystal was used. First the measured and calculated values will be defined for the calculations.

$$
\mathrm{R}_{1}:=0.978 \quad \mathrm{R}_{2}:=0.9985 \quad \gamma_{\mathrm{SH}}:=0.0131 \quad \mathrm{~T}_{1}:=1-\mathrm{R}_{1} \quad \mathrm{t}:=0.975
$$

Here $R_{1}$ is the cavity input mirror power reflection coefficient, $\mathbf{R}_{\mathbf{2}}$ is the cavity output mirror power reflection coefficient, $\gamma_{\mathrm{SH}}$ is the nonlinear conversion factor for the $7.5 \mathrm{~mm}$ $\mathrm{KNbO}_{3} \mathrm{NLO}$ crystal, $\mathrm{T}_{1}$ is the input mirror power transmission coefficient (assuming lossless mirrors) and $t$ is the single-pass linear loss within the cavity. Now the measured input fundamental powers $\mathrm{Pfund}_{\mathrm{i}}$ and corresponding $\mathrm{SH}$ output powers $\mathrm{PSH}_{\mathrm{i}}$ from the cavity will be input to arrays for calculations.

$$
\begin{aligned}
& \text { i }:=1 . .6 \quad \text { PSH }_{i}:=\quad \text { Pfund }_{i}:= \\
& \begin{array}{|l|l|}
\hline 0.00275 \\
\hline 0.0031 \\
\hline 0.0039 \\
\hline 0.00505 \\
\hline 0.00645 \\
\hline 0.0078 \\
\hline
\end{array} \quad \begin{array}{l|l|}
\hline 0.031 \\
\hline 0.0349 \\
\hline 0.039 \\
\hline 0.043 \\
\hline 0.0505 \\
\hline 0.0596 \\
\hline
\end{array}
\end{aligned}
$$

Since the SH powers are those measured from one side of the cavity, the circulating power within the cavity can be determined using 


$$
\mathrm{Pc}_{\mathrm{i}}:=\sqrt{\frac{\mathrm{PSH}_{\mathrm{i}}}{\gamma \mathrm{SH}}}
$$

Using this relationship, the circulating powers within the cavity are determined to be

$\mathrm{Pc}_{\mathrm{i}}$
\begin{tabular}{|l|}
\hline 0.4582 \\
\hline 0.4865 \\
\hline 0.5456 \\
\hline 0.6209 \\
\hline 0.7017 \\
\hline 0.7716 \\
\hline
\end{tabular}

where each of these values corresponds to the fundamental input powers described above and in the same array position. Now the cavity reflectance parameter (using Eq. 4 from Appendix A) will be defined in terms of the calculated circulating fundamental power within the cavity and $\backsim$ aluated. Note that there are two solutions to this relationship, however only one is valid.

$$
\begin{aligned}
& \mathrm{rm}_{\mathrm{i}}:= \frac{\left(1-\sqrt{\frac{\mathrm{T}_{1} \cdot \text { Pfund }_{\mathrm{i}}}{\mathrm{Pc}_{\mathrm{i}}}}\right)^{2}}{\mathrm{R}_{1}} \\
& \frac{\mathrm{rm} 2_{\mathrm{i}}}{0.9451} \\
& \hline \frac{0.9429}{0.943} \\
& \hline 0.9442 \\
& \hline 0.9427 \\
& \hline 0.9399 \\
& \hline
\end{aligned}
$$

Then using the calculated values for $\mathrm{rm} 2_{i}$ (these are the valid solutions), the predicted reflectance of the fundamental beam incident onto the cavity will be determined. These values will then be compared to measured reflectance values from the cavity. The reflectance from the cavity and its solutions are given by 


$$
\operatorname{Prefl}_{i}:=\frac{\left(\sqrt{R_{1}}-\sqrt{r m 2_{i}}\right)^{2}}{\left(1-\sqrt{R_{1} \cdot \mathrm{rm} 2_{i}}\right)^{2}}
$$

Prefl $_{i}$
\begin{tabular}{|l|}
\hline 0.1889 \\
\hline 0.2036 \\
\hline 0.2027 \\
\hline 0.1947 \\
\hline 0.2044 \\
\hline 0.2223 \\
\hline
\end{tabular}

Now, using the calculated values for $\mathrm{rm} 2_{\mathrm{i}}$, the expected value for the circulating power will be calculated when the input mirror reflectivity is reduced to 0.9444 (a mirror which we presently possess). This mirror reflectivity should provide a better impedance-match to the cavity. Based on the values obtained, the expected SH output from the cavity will then be determined. These values will then be compared to the measured SH values obtained from the cavity.

$$
\mathrm{Pcl}_{\mathrm{i}}:=\frac{0.0556 \cdot \text { Pfund }_{\mathrm{i}}}{\left(1-\sqrt{\left.0.9444 \cdot \mathrm{mm} 2_{\mathrm{i}}\right)^{2}}\right.}
$$

\begin{tabular}{ll|}
$\mathrm{Pcl}_{\mathrm{i}}$ & $\mathrm{Pc}_{\mathrm{i}}$ \\
\hline 0.5648 & 0.4582 \\
\hline 0.6107 & \\
\hline 0.6842 \\
\hline 0.771 \\
\hline 0.8818 \\
\hline 0.9907 \\
& 0.5465 \\
\hline & 0.6209 \\
\hline & 0.7017 \\
\hline & 0.7716 \\
\hline
\end{tabular}

Now the expected $\mathrm{SH}$ output power $\mathrm{PSH}_{\mathrm{i}}$ when the input mirror reflectivity is reduced will be calculated based on these predicted circulating powers $\operatorname{Pcl}_{\mathbf{i}}$. These values will then be compared to the experimentally determined values obtained using the higher input mirror reflectivity. 


\begin{tabular}{|c|c|}
\hline $\mathrm{PSH1}_{\mathrm{i}}$ & $\mathrm{PSH}_{\mathrm{i}}$ \\
\hline 0.0042 & 0.0027 \\
\hline 0.0049 & 0.0031 \\
\hline 0.0061 & 0.0039 \\
\hline 0.0078 & 0.005 \\
\hline 0.0102 & 0.0065 \\
\hline 0.0129 & 0.0078 \\
\hline
\end{tabular}

Comparison of the predicted SH values when input mirror reflectivity is reduced with the measured SH values with the higher input mirror reflectivity indicates that a factor of 1.6 increase in the SH output power is expected by reducing the input mirror reflectivity to 0.9444 . 


\section{REFERENCES}

1. Cooper LaserSonics, Inc., 928 E. Meadow Drive, Palo Alto, Ca. 94303 - CW Ion Lasers Specification Pamphlet.

2. D. C. Gerstenberger, G. E. Tye and R. W. Wallace, "Efficient second-harmonic conversion of $\mathrm{cw}$ single-frequency Nd:YAG laser light by frequency locking to a monolithic ring frequency doubler," Opt. Lett. 16:992, 1991.

3. A. Yariv, "Optical Electronics," Saunders College Publishing, Philadelphia, 1991.

4. P. A. Franken, A. E. Hill, C. W. Peters and G. Weinreich, "Generation of Optical Harmonics," Phys. Rev. 127:1918, 1962.

5. R. G. Smith, "Theory of Intracavity Optical Second-Harmonic Generation," IEEE J. Quant. Elec. QE-6:215, 1970.

6. P. E. Perkins and T. S. Fahlen, "20-W average-power KTP intracavity-doubled Nd:YAG laser," J. Opt. Soc. Am., B. 4:1066, 1987.

7. A. Ashkin, G. D. Boyd and J. M. Dziedzic, "Resonant Optical Second Harmonic Generation and Mixing," IEEE J. Quantum Electron. QE-2:109, 1966.

8. S. T. Yang, C. C. Pohalski, E. K. Gustafson, R. L. Byer, R. S. Feigelson, R. J. Raymakers and R. K. Route, "6.5-W, 532-nm radiation by $\mathrm{CW}$ resonant externalcavity second-harmonic generation of an 18-W Nd:YAG laser in $\mathrm{LiNb}_{3} \mathrm{O}_{5}, "$ Opt. Lett. 16:1493, 1991. 
9. W. J. Kozlovsky, "Frequency-Doubled Diode Lasers," Compact Blue-Green Lasers, 1992 Technical Digest Series, v. 6, (Optical Society of America, Santa Fe, New Mexico)p. 91.

10. J. Golden, "Green Lasers Score Good Marks in Semiconductor Material Processing," Laser Focus World, 28:75, June 1992.

11. M. V. Klein and T. E. Furtak, "Optics," John Wiley and Sons, New York, 1986.

12. A. E. Siegman, "New developments in laser resonators," SPIE Proc. vol. 1224 Optical Resonators p. 2, 1990.

13. M. W. Sasnett and T. F. Johnston, "Beam characterization and measurement of propagation attributes," SPIE Vol. 1414 Laser Beam Diagnostics p.21, 1991.

14. A. E. Siegman, "Lasers," University Science Books, Mill Valley, 1986.

15. H. Kogelnik and T. Li, "Laser Beams and Resonators," Appl. Opt. 5:1550, 1966.

16. R. L. Fork, D. R. Herriott and H. Kogelnik, "A Scanning Spherical Mirror Interferometer for Spectral Analysis of Laser Radiation," Appl. Opt. 3:1471, 1964.

17. R. W. Boyd, "Nonlinear Optics," Academic Press, Boston, 1992.

18. G. D. Boyd and D. A. Kleinman, "Parametric Interaction of Focused Gaussian Light Beams," J. Appl. Phys. 39:3597, (1968). 
19. W. J. Kozlovsky, C. D. Nabors and R. L. Byer, "Efficient Second Harmonic Generation of a Diode-Laser-Pumped CW Nd:YAG Laser Using Monolithic MgO:LiNbO 3 External Resonant Cavities," IEEE J. Quantum Electron. QE24:913, 1988.

20. Spectra Diode Labs, 80 Rose Orchard Way, San Jose, Ca. 95134, "Laser Diode Operator's Manual \& Technical Notes".

21. MPB Technologies Inc. 1725 No. Service Rd., Trans Canada Hwy, Dorval, Quebec, H9P 1J1, Canada, Laser Stabilization Device Manual.

22. R. L. Lang and K. Kobayashi, "External Optical Feedback Effects on Semiconductor Injection Laser Properties," IEEE J. Quantum Electron. QE-16, 347 (1980).

23. B. Dahmani, L. Hollberg and R. Drullinger, "Frequency stabilization of semiconductor lasers by resonant optical feedback," Opt. Lett. 12, 876, (1987).

24. Merchantek, Inc., 12526 High Bluff Dr., San Diego, Ca. 92130, "Beamscope User Manual".

25. Newport Corp., 18235 Mt. Baldy Cir., Fountain Valley, Ca. 92708, PID Temperature Controller Manual

26. CVI Laser Corp., 200 Dorado Place, SE, Albuquerque, N. M. 87192, Reflectivity curves supplied with optics. 
27. Virgo Optics, 6736 Commerce Ave., Port Richey, Fl. 33568, Reflectivity curves supplied with $\mathrm{KNbO}_{3} \mathrm{NLO}$ crystal.

28. ILX Lightwave Corp., Box 6310, Bozeman, Mt. 59771, Calibration curve supplied with thermistor.

29. J. C. Baumert, J. Hoffnagle and P. Günter, "Nonlinear optical effects in $\mathrm{KNbO}_{3}$ crystals at $\mathrm{Al}_{\mathrm{x}} \mathrm{Ga}_{1-\mathrm{x}} \mathrm{As}$, dye, ruby and Nd:YAG laser wavelengths," SPIE Proc. 492, 374 (1984).

30. T. L. Lucas, (Private Communication)

31. J. T. Lin and S. W. Chong, "Large-signal Theory for Frequency-mixing in Diodepumped Q-switched Systems," SPIE Proc. 1040, 191 (1989). 


\section{DISTRIBUTION:}

1 T. L. Luias

University of New Mexico

Electrical and Computer Engineering Dept.

EECE Bldg., rm. 110

Albuquerque, N. M. 87131

1 Hua Li

University of New Mexico

Electrical and Computer Engineering Dept.

EECE Bldg., rm. 110

Albuquerque, N. M. 87131

1 Greg Mizell

Virgo Optics

6736 Commerce Ave.

Port Richey, Fl. 34668

1 Carl Miller

U. S . Laser Corp.

825 Windham Court North

Wyckoff, N. J. 07481-0609

$1 \quad 1128 \quad$ G. N. Hays

$\begin{array}{lll}1 & 1128 & \text { T. D. Raymond }\end{array}$

$1 \quad 1128 \quad$ W. J. Alford

$1 \quad 1128$ A. V. Smith

$1 \quad 1800 \quad$ A. D. Romig

$1 \quad 1831 \quad$ M. J. Cieslak

$\begin{array}{lll}10 & 1831 & \text { D. M. Keicher }\end{array}$

$\begin{array}{lll}1 & 1831 & \text { G. A. Knorovsky }\end{array}$

$1 \quad 1833 \quad$ J. L. Jellison

$12514 \quad$ K. J. Fleming

1 8523-2 Central Technical Files

57141 Technical Library

17151 Technical Publications

10 7613-2 Document Processing for DOE/OSTI 


\section{$Y$}
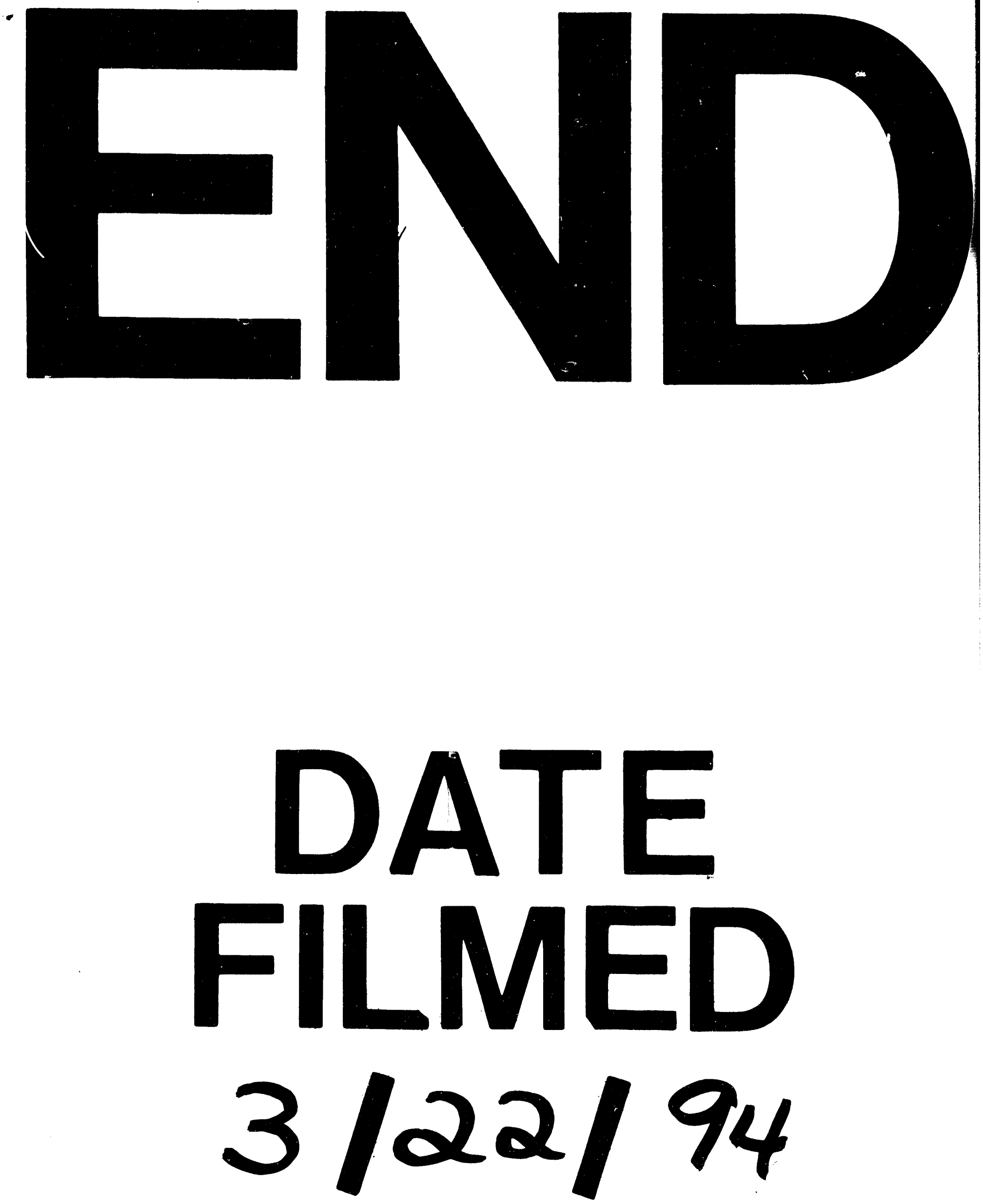
